University of Louisville

ThinkIR: The University of Louisville's Institutional Repository

Electronic Theses and Dissertations

$5-2004$

\title{
The visual perception of distance in action space.
}

Bing Wu 1973-

University of Louisville

Follow this and additional works at: https://ir.library.louisville.edu/etd

\section{Recommended Citation}

Wu, Bing 1973-, "The visual perception of distance in action space." (2004). Electronic Theses and Dissertations. Paper 1598.

https://doi.org/10.18297/etd/1598

This Doctoral Dissertation is brought to you for free and open access by ThinkIR: The University of Louisville's Institutional Repository. It has been accepted for inclusion in Electronic Theses and Dissertations by an authorized administrator of ThinkIR: The University of Louisville's Institutional Repository. This title appears here courtesy of the author, who has retained all other copyrights. For more information, please contact thinkir@louisville.edu. 


\title{
THE VISUAL PERCEPTION OF DISTANCE IN ACTION SPACE
}

\author{
By \\ Bing Wu \\ B.S., Huazhong University of Technology \& Sciences, 1994 \\ M.A., Shanghai Institute of Physiology, 1997
}

\begin{abstract}
A Dissertation
Submitted to the Faculty of the

Graduate School of the University of Louisville

in Partial Fulfillment of the Requirements

for the Degree of

Doctor of Philosophy

Department of Psychological \& Brain Sciences

University of Louisville

Louisville, Kentucky
\end{abstract}

May 2004 
THE VISUAL PERCEPTION OF DISTANCE IN ACTION SPACE

By

Bing Wu

B.S., Huazhong University of Technology \& Sciences, 1994

M.A., Shanghai Institute of Physiology, 1997

A Dissertation Approved on

April 16, 2004

by the following Dissertation Committee:

Dissertation Director 


\section{ACKNOWLEDGMENTS}

I would like to thank all the people who helped me during my time as a Ph.D. student at the University of Louisville.

First of all, I would like to express my gratitude to my advisor, Professor Zijiang He, for his guidance and patience throughout my studies. Without help and support from him, I would never have been able to finish this work.

I would like to thank Professors Frederic Wightman, John Pani, Pavel Zahorik, and Dar-jen Chang for serving in my defense committee, Professor Heywood Petry for serving in my qualification committee, and Professor Edward Essock for serving both committees.

I would also give thanks to Lei Wang, Jun Wu, Gary Yarbrough, and Yong Su for the valuable collaboration.

Also, many thanks to the subjects who participated in my experiments.

Finally, my love goes to my parents for all their support and encouragement that kept me going. 


\title{
ABSTRACT \\ THE VISUAL PERCEPTION OF DISTANCE IN ACTION SPACE
}

\author{
Bing Wu
}

May 8, 2004

This work examines our perception of distance within action space (about $2 \mathrm{~m} \sim$ $30 \mathrm{~m}$ ), an ability that is important for various actions. Two general problems are addressed: what information can be used to judge distance accurately and how is it processed? The dissertation is in two parts.

The first part considers the "what" question. Subjects' distance judgment was examined in real, altered and virtual environments by using perceptual tasks or actions to assess the role of a variety of intrinsic and environmental depth cues. The findings show that the perception of angular declination, or height in the visual field, is largely veridical and a target is visually located on the projection line from the observer's eyes to it. It is also shown that a continuous ground texture is essential for veridical space perception. Of multiple textural cues, linear perspective is a strong cue for representing the ground and hence judging distance but compression is a relatively ineffective cue.

In the second part, the sequential surface integration process (SSIP) hypothesis is proposed to understand the processing of depth information. The hypothesis asserts that an accurate representation of the ground surface is critical for veridical space perception 
and a global ground representation is formed by an integrative process that samples and combines local information over space and time. Confirming this, the experiments found that information from an extended ground area is necessary for judging distance accurately and distance was underestimated when an observer's view was restricted to the local ground area about the target. The SSIP hypothesis also suggests that, to build an accurate ground representation, the integrative process might start from near space where rich depth cues can provide for a reliable initial representation and then progressively extend to distant areas. This is also confirmed by the finding that subjects could judge distance accurately by scanning local patches of the ground surface from near to far, but not in the reverse direction. 


\section{LIST OF FIGURES}

FIGURES

PAGE

1. Scheme for computing absolute distance ................................. 113

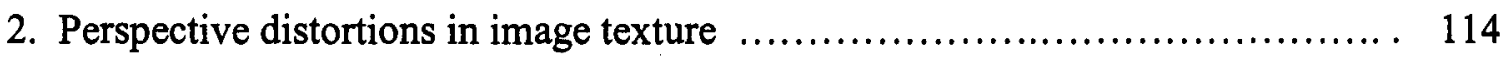

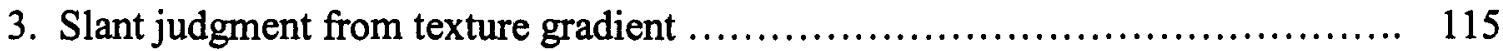

4. Distance judgment in the cue-reduced dark environment $\ldots \ldots \ldots \ldots \ldots \ldots \ldots \ldots \ldots, 116$

5. Linear mapping between verbal reports and walked distances................. 117

6. Distance and height judgment in the lit environment ........................ 118

7. The perception of angular declination in the lit environment .................. 119

8. Distance and height judgment in the dark environment ...................... 120

9. The perception of angular declination in the dark environment $\ldots \ldots \ldots \ldots \ldots \ldots \ldots, 121$

10. Variance in the perceived angular declination ............................ 122

11. Perceived locations of the target in the prism study ........................ 123

12. Judged angular declination and eye level in the prism study.................. 124

13. Distance judgment with reference to a textured ground in the dark ............. 125

14. Reliability analysis of the verbal estimation protocol f........................ 126

15. Influence of false perspective cue on distance judgment .................... 127

16. Ineffective compression cue on distance judgment......................... 128

17. Ineffective false compression cue on distance judgment ..................... 129 
18. High efficacy of perspective cue on distance judgment

19. The texture-location effect on distance judgment........................... 131

20. False perspective effect examined using the blindfolded throwing ............. 132

21. Misrepresentation of the ground due to false perspective information

22. Misrepresentation of the ground and eye level shift caused by false perspective . 134

23. The Sequential Surface Integration Process (SSIP) hypothesis

24. Accurate surface representation from the SSIP process

25. The critical role of the near ground information

26. The continuity assumption

27. Settings for the texture-discontinuity experiment in VR

28. Results of the texture-discontinuity experiment in VR

29. Distance underestimation due to discontinuity in the ground texture.

30. Distance underestimation in the presence of texture discontinuity

31. Frontal-shift error in the ground representation due to the texture discontinuity..

32. Settings for the occlusion experiment in VR

33. Results of the occlusion experiment in VR.

34. Settings and results of the wall-behind-target control experiment

35. The filed-of-view (FOV) -restricting goggles

36. Absolute distance judgment from a static view with the restricted FOV

37. Relative distance judgment from a static view with the restricted FOV

38. Slant errors from blindfolded walking and L-shape matching tasks

39. Distance judgment from a static view with a vertically-restricted FOV 
41. Distance judgment with head motion in the FOV-restriction condition

42. Directional asymmetry in distance judgment

43. Distance judgment with two directional scans

44. The control experiment in the dark

45. The Oppel-Kundt illusion

46. The texture discontinuity effect in the frontal plane

47. Influence of perspective information on the discontinuity effect

48. Distance judgment across different number of boundaries

49. An explanation for the discontinuity effect

50. Distance judgment with different extent of the occlusion 162 


\section{TABLE OF CONTENTS}

PAGE

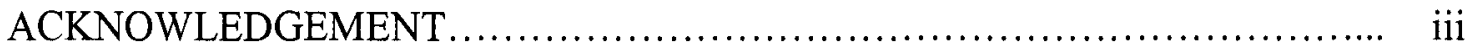

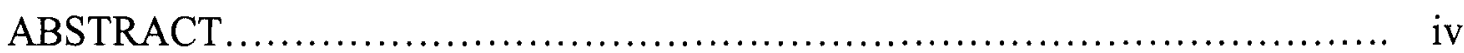

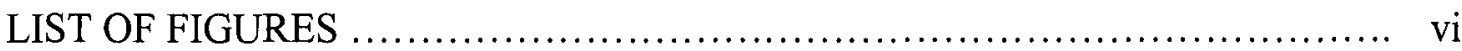

Chap1. Introduction ...................................................... 1

1.1 The perception of egocentric and exocentric distances................... 1

1.2 Theoretical approaches to distance perception........................... 3

1.3 Information for judging distance...................................... 7

1.4 Visual texture information for representing a surface..................... 8

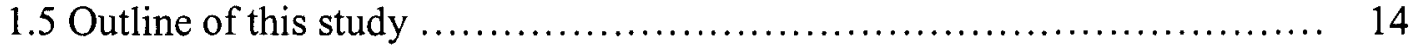

Chap 2. Direction constancy in space perception $\ldots \ldots \ldots \ldots \ldots \ldots \ldots \ldots \ldots \ldots \ldots \ldots$

2.1 Exp 2.1. Influence of angular declination information on distance judgment. 16

2.2 Exp 2.2. Judging the target's angular declination in the lit environment $\ldots \ldots .21$

2.3 Exp 2.3. Judging the target's angular declination in the dark .............. 23

2.4 Direction constancy in space perception ............................. 25

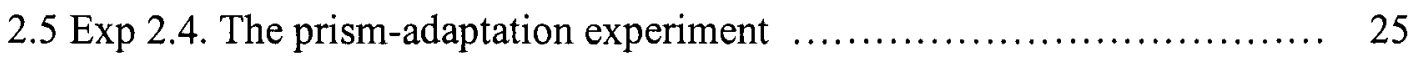

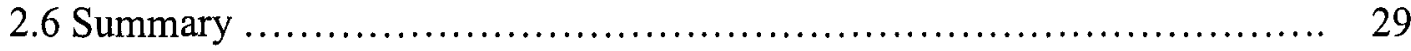

Chap 3. Effectiveness of texture cues in distance perception ................... 30

3.1 Exp 3.1. Distance judgment with a continuous ground texture ............. 31 
3.2 Exp 3.2. The effect of false linear perspective on distance perception.

3.3 Exp 3.3. Relative ineffectiveness of texture compression cue ............... 36

3.4 Exp 3.4. Little to no effect of false compression cue on distance perception.. 38

3.5 Exp 3.5. High efficiency of linear perspective ............................ 39

3.6 The texture location effect reexamined................................ 41

3.7 Exp 3.6. The false perspective effect examined using blindfolded throwing.. 41

3.8 Exp 3.7. Eye height shift in the presence of false linear perspective ......... 44

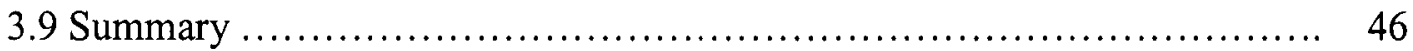

Chap 4. Sequential surface integration process hypothesis ..................... 48

4.1 Space perception: an integrative process............................ 49

4.2 The Sequential Surface Integration Process (SSIP) hypothesis $\ldots \ldots \ldots \ldots \ldots . \quad 51$

4.3 Predictions from the SSIP hypothesis............................. 54

4.4 The plan to assess the SSIP hypothesis.............................. 56

Chap 5. Ground-based distance perception in virtual reality ..................... 58

5.1 The virtual reality system and virtual environments ..................... 59

5.2 Exp 5.1. Distance underestimation across discontinuous textures ............ 60

5.3 Exp 5.2. The ground representation errors due to texture discontinuity...... 62

5.4 Exp 5.3. The occlusion effect on distance judgment .................... 66

5.5 Exp 5.4. SSIP or "equidistance tendency"? ........................... 68

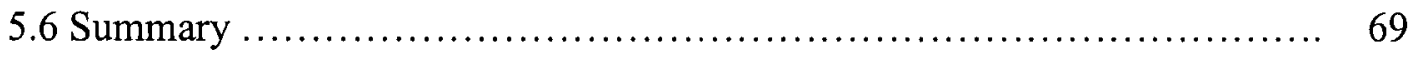

Chap 6. Ground representation from an integrative process ........................ 70

6.1 Exp 6.1. Absolute distance judgment with restricted FOV ................. 71

6.2 Exp 6.2. Relative distance judgment with restricted FOV .................. 74 
6.3 Exp 6.3. Judging distances with vertically restricted FOV

6.4 Exp 6.4. Judging distances with horizontally narrowed FOV ............... 79

6.5 Exp 6.5. Accurate distance judgment with free head movement ........... 80

6.6 Exp 6.6. The directional asymmetry in distance judgment ................ 81

6.7 Exp 6.7. Distance judgment with two scans .......................... 83

6.8 Exp 6.8. Distance judgment in the dark with or without head motion ........ 85

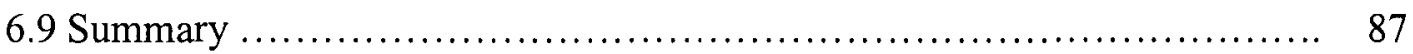

Chap 7 Hypotheses about the texture-discontinuity effect....................... 89

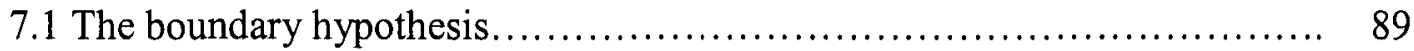

7.2 Comments on two hypotheses...................................... 91

7.3 Exp 7.1. The 2D texture-discontinuity effect $\ldots \ldots \ldots \ldots \ldots \ldots \ldots \ldots \ldots \ldots . \ldots 2$

7.4 Exp 7.2. Influence of perspective information on the discontinuity effect .... 94

7.5 Exp 7.3. Distance judgment across different number of boundaries ......... 96

7.6 Exp 7.4. Influence of amount of occlusion on the occlusion effect ......... 99

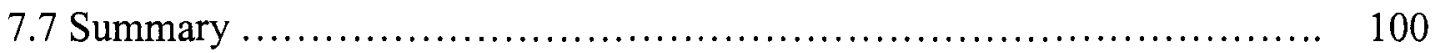

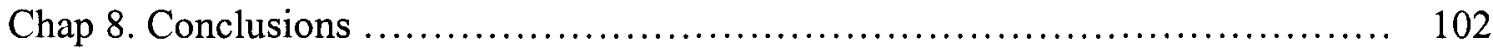

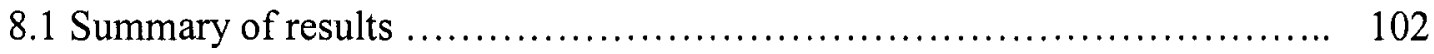

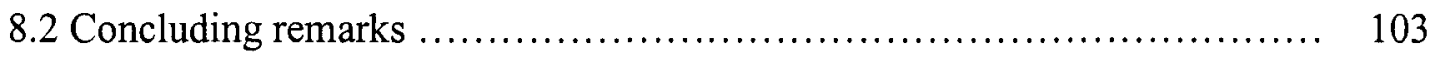

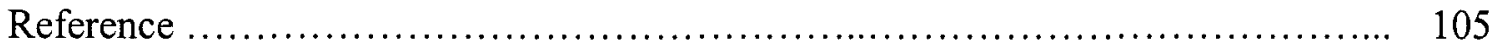

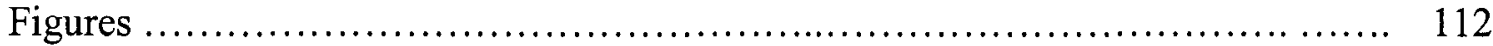

Curriculum Vitae ............................................................ 163 


\section{CHAPTER 1}

\section{INTRODUCTION}

The goal of vision is to provide the animal with a representation of the external world so that it can interact with the environment about it. To build up such a representation, the visual system needs to discover what are present in the world and where they are. The current work concerns the "where" question. The aim is to investigate how people make judgments about distance within action space (defined by Cutting \& Vishton (1995) as the region surrounding a viewer from about $2 \mathrm{~m}$ to $30 \mathrm{~m}$ ), which is believed to be important to the control of spatially related behavior. The general problems addressed are: what depth information can be used for judging distance accurately and how is it processed?

\subsection{The perception of egocentric and exocentric distances}

Distance estimation can be egocentric (i.e., absolute distance from the observer to a target) or exocentric (i.e., relative distance between two objects). For egocentric distance perception, investigators have described the relation between judged distance, $D^{\prime}$, and actual distance, $D$, as a power function: $D^{\prime}=k D^{n}$, where $k$ is a scale factor that depends on the unit of measurement and the exponent $n$ indicates the nature of the visual space. When $n$ is 1 , the visual space is intrinsically Euclidean and the relation between 
the perceived and physical distance is linear; over- or under-constancy is indicated by $\mathrm{n}>1$ and $\mathrm{n}<1$, respectively. Most previous studies found constancy or under-constancy of egocentric distance perception ( $n \approx 1$ : Purdy \& Gibson, 1955; Vincent, et al. 1968; Cook, 1978; Wagner, 1985; $n<1$ : Gilinsky, 1951; Kunnapas, 1968; Teghtsoonian \& Teghtsoonian, 1970; Hagen \& Teghtsoonian, 1981; Da Silva, 1982a-b). The reported exponent varies between 0.91 and 1.00 (for reviews see Wiest \& Bell, 1985; Sedgwick, 1986), depending on the interactions among the range of distances, the experimental environment, the methods for obtaining distance judgments and the viewing conditions.

In general, under natural, unrestricted viewing conditions, people are remarkably accurate in judging egocentric distance in the intermediate range and the $n$ approximates 1.0. Some studies, based on subjects' motoric responses to visual targets, show that there is little to no systematic error in the perception of distance up to about $24 \mathrm{~m}$ (Thompson, 1983; Eby \& Loomis, 1987; Elliott, 1986, 1987; Rieser et al. 1990; Loomis et al. 1992; Loomis et al. 1996; Philbeck \& Loomis, 1997; Loomis, 1999). Take the blindfoldedwalking as the example. When people are asked to view an object and then walk to it with eyes closed, they can correctly arrive at the location (Thompson, 1983; Rieser et al. 1990; Loomis et al. 1992; Loomis, 1999).

The perception of exocentric distance, on the other hand, shows perceptual foreshortening in the depth (Glinsky, 1951; Harway, 1963; Toye, 1986; Levin \& Haber, 1993; Loomis et al., 1996; Loomis \& Philbeck, 1999). For example, Glinsky (1951) asked subjects to mark off successive increments of 1 feet or 1 meter extending away from them. The first several increments were fairly accurate, but larger and larger intervals were marked as the increment moved away from the observer. A further 
illustration of perceptual compression in depth is the anisotropy in exocentric distance judgment. The same physical distance is seen longest in the frontal orientation, gradually contracted when oriented, and shortest in depth (Wagner, 1985). Loomis et al. (1992) reported that intervals in depth had to be set to $50 \%$ to $90 \%$ more than frontal intervals to appear equal, although an objective instruction told their subjects to match physical distances.

\subsection{Theoretical approaches to distance perception}

How does the visual system judge distance in action space? The question is considered here from three theoretical approaches: the cue approach, the "ground theory" proposed by J.J. Gibson (1950a, 1979), and Marr's computational theory (1982).

\section{The cue theory}

The cue approach focuses on identifying different sources of information, called depth cues, that are correlated with depth in the environment and enable us to make inferences about distance. A number of cues for absolute and relative distance have been identified. They are divided into four types (Gillam 1995; Goldstein 1996):

Oculomotor cues (convergence and accommodation): The object, when fixated, is located in space using the position of eyes and the proprioceptive feedback from eye muscles.

Binocular disparity: The difference between the images produced in the left and right eye provides depth information.

Motion-produced cue: When the observer moves, objects are seen to move in different directions and speeds (motion parallax). Also, distant objects can be hidden or 
exposed by closer objects (deletion and accretion) from different viewpoints. Relative depth thus is signaled.

Pictorial depth cues: Artists use these cues to capture the 3D structure of a scene in a 2D image. Examples of pictorial cues are occlusion, perspective, height in the field of view, relative size, texture gradient, shading and shadow, and so on.

It should be noted that not all depth cues are operative within action space because most non-pictorial cues reduce their effectiveness rapidly with increasing distance and have limited effective ranges. For example, the effectiveness of oculomotor cues and absolute motion perspective is confined to a range up to about $2 \sim 3 \mathrm{~m}$. In contrast, pictorial cues generally preserve their strength at long distances. Thus there is a general shift toward reliance on pictorial cues with distance. Accordingly, it has been suggested that the operative depth cues within action space, ranked by their relative effectiveness, are occlusion, height in the field of view, motion parallax, relative size, binocular disparity, and texture gradient (Nagata, 1991; Cutting \& Vishton, 1995).

Of six depth cues, height in the field of view may be especially important for judging egocentric distance. According to Gogel's classification, other five cues are relative depth cues that indicate "one object is more distant that another (a perception of exocentric distance) without specifying the apparent distance of either object from the observer" (p137, Gogel, 1977). For example, occlusion is a very reliable cue to relative depth, but it provides no information about absolute distance. In contrast, height in the field of view, measured by angular declination from the eye level, can determine the absolute distance of a single object from the viewer (Wallach \& O'Leary 1982; Levin \& Haber, 1993; Philbeck \& Loomis, 1997; Wu et al., 2000; Ooi et al., 2001). In theory, with 
reference to a level ground, distance to a target can be computed by dividing the observer's eye height by the tangent of angular declination (Figure 1.1(a)).

\section{Gibson's "ground theory" of space perception}

Gibson was the first to suggest that space perception is reducible to the perception of visual surfaces. According to his "ground theory" of space perception (1950a, 1979), the perceived space is not an empty, abstract geometrical space. Instead, Gibson argues that the visual space is conceived as an array of surfaces and hence the recovery of distance or depth information is the consequence of surface perception. Of all surfaces in the environment, the ground is considered as "the most important of all surfaces for terrestrial animals. ... It is the ground of their perception and behavior, both literally and figuratively" (p16, Gibson, 1979). Therefore, "the problem of three-dimensional vision is basically a problem of the perception of a continuous surface which is seen to extend away from the observer" (p420, Gibson, 1946) and distance is perceived as "recession along the ground".

Rather than attempting to understand how the visual system might recover distances to points in the visual field, Gibson emphasizes how the spatial properties of the visible surfaces are perceived. He suggests that the optical array carries sufficient information about surface layout. Spatial qualities such as surface shape, orientations and distances can be directly derived from invariants or 'constant patterns of variation' in the optic stimuli, which could be picked up and determine our perception uniquely. For example, information in the texture image, i.e. texture gradients, is such a powerful invariant. "The apparent slant of a surface to the line of regard at any point might be given by the rate of increase of the density of elements at the corresponding point in the 
image. The direction of the slant would correspond to the direction of the gradient" (p373, Gibson, 1950b), and "Distance may be given by the relative density of texture, the finer the density the greater being the distance" (p371, Gibson, 1950b).

\section{Marr's computational theory}

The cue approach focuses on identifying information in the retinal image that is correlated with depth while Gibson aims to find out what in the environment makes perception possible. Unlike these two approaches, Marr's theory (1982) regards vision as information processing and the emphasis has shifted to understanding the nature of information process.

Marr sees vision as proceeding by the explicit computation of a sequence of representations. Taking a bottom-up computational approach, his model starts with more or less local computations. The primal sketch is derived first, which describes local intensity changes in the image and their geometrical distribution and organization. The subsequent step is to reconstruct a viewer-centered representation of visible surfaces and their characteristics $\left(2 \frac{1}{2}\right.$-D sketch). Information from different parts of the image or different cues at the same location like stereopsis, shading, texture all contributes to representing the $2 \frac{1}{2}-\mathrm{D}$ sketch, which is next transformed into a $3 \mathrm{D}$ object-centered representation.

In this computational frame, surface representation is one product of visual processing. The representation is "not only to make explicit information about depth, local surface orientation, discontinuities in these quantities but also to create and maintain a global representation of depth that is consistent with the local cues that these sources 
provide" (p277, Marr, 1982). Following this idea, space perception is thus understood by discovering how a global representation of the ground surface is computed.

\subsection{Information for judging distance}

The above analysis highlights the role of the ground representation and angular declination in distance perception. In theory, for a target resting on the ground, the distance to it can be computed by using information about its angular declination $(r)$, the observer's eye height $(h)$, and the geographical slant of the ground $(\theta)$. As shown in Figure 1.1(a), distance can be computed by using the equation: $d=h / \tan (r)$ if an upright posture and a level ground are assumed; distance on an upward slope can be computed by using the equation: $d=h^{*} \cos (\gamma) / \sin (\gamma+\theta)$, if the slant of the ground slope $(\theta)$ has accurately judged (Figure 1.1(b)).

To be effective cues for distance, these variables $(r, h, \theta)$ need to be perceived accurately by the visual system. Let's consider the perception of eye height first. Previous research has shown that people can judge their eye height with reasonable accuracy. For example, Stoper \& Cohen (1986) found that the visually perceived eye level in the lit environment matches perfectly with the physical value $\left(-0.29^{\circ} \pm 1.03^{\circ}\right)$. Sinai et al. (1998) explored how distance judgment was influenced by the perceived eye height. Their subjects stood on a level ground or an elevated platform in the experiment. The judgment of distance and eye height was assessed using a perceptual matching paradigm. The results found that distance and eye height could be accurately judged in the stand-onthe-ground condition. But when the observer stood on an elevated platform, the eye height with reference to the lower ground was perceptually exaggerated. Distance on the 
ground was also overestimated in this condition. More important, distance overestimation could be quantitatively related to the exaggerated perception of eye height. The finding thus supports the idea that the visual system can accurately register the eye height information and use it for judging distance (Mark, 1987; Warren, \& Whang, 1987; Sinai et al., 1998).

As to the perception of angular declination, this issue will be addressed in the current study. In Chapter 2 subjects' perception of angular declination will be measured in both dark and lit environments.

Afterward, the process of representing the ground will be examined. Both "what" and "how" questions will be discussed in Chapters 3 7: What information is useful for representing the ground and accordingly supplying distance perception, and how does the brain build an accurate ground representation from the information? It should be noted that texture is a significant source of information for representing the surface (Gibson, 1950a-b, 1979; Prudy, 1960; Stevens, 1979; Cutting \& Millard 1984; Sedgwick, 1986; Knill, 1998a-c). A continuous and homogeneous ground texture is believed to be vital for the visual system to form an accurate ground surface representation (Sinai et al., 1998). So the following section will move on to briefly review the information content of texture.

\subsection{Visual texture information for representing a surface}

For simplicity, texture is defined here as the more or less regular repetition of a basic pattern on the surface, for example, tiles on the indoor floor or grass clumps on the outdoor ground. 
Although surface texture distribution is usually homogenous or isotropic, its appearance in the image presents inhomogeneity or/and anisotropy owing to projective distortions caused by changes in distance or surface orientation. As shown in the Figure 1.2(a), perspective projection distorts the appearance of an object in two ways: by scaling its apparent size by $1 / D$ and by foreshortening its apparent shape vertically by $\cos (\delta)$ (Stevens, 1979, 1981; Todd \& Akerstrom, 1987; Knill, 1992, 1998a-c). When applied to a textured surface, it distorts the appearance of individual texture elements as well as the structure of texture pattern. In Figure 1.2(b), disks on the ground look more elliptical and smaller with increasing distance. Apparent spacing among disks also gradually decreases. This introduces three depth cues: an increase in texture density, a compression of depth intervals among disks, and linear perspective (convergence of lines of disks). Theoretically, all these distortions - increased density, deceased spacing, flattened shape of texture elements and linear perspective - enable the visual system to infer 3D configurations from 2D images.

\section{Information content of texture gradients}

If texture is continuously distributed in depth, perspective distortions in the image can be measured by the rate of change, texture gradient. Given that perspective projection changes the size, shape, and density of texture elements, texture gradients are characterized by the measure of corresponding distortions: density gradient, perspective gradient, and compression gradient. Mathematically, there are two definitions for texture gradient. Stevens' definition (1979) uses the direct measure of the rate of change as $\nabla x=\left(\frac{d x}{d \delta}\right)$, where $x$ is the measure of projective distortions and $\delta$ is the optical slant, the inclination between the line of sight and the surface normal (For definition, see Figure 
1.3a). Purdy's definition (1960) employed the normalized rate of change: $G_{x}=\left(\frac{d x}{d r}\right) / x$, where $r$ is the complementary angle of optical slant at that location $\left(r=90^{\circ}-\delta\right)$. Table 1-1 summarizes two definitions. Clearly, there exists a correspondence between texture gradient and surface orientation: $G_{x} \propto \cot r$ in Purdy's definition and $\nabla x \propto \sin \delta$ for gradient of linear perspective and compression in Stevens' definition. As a result, surface slant $(\delta)$ can be directly inferred from texture gradient by $\delta=\arctan \left(G_{x} / k\right)$ or $\delta=\arcsin (\nabla x / k)$, where $k$ is a constant and its value depends on the measure of perspective distortions and the definition of texture gradient.

Table 1-1. Texture gradients in different definition

\begin{tabular}{|c|c|c|c|}
\hline $\begin{array}{c}\text { Measure of } \\
\text { distortion }\end{array}$ & $\begin{array}{c}\text { Purdy's } \\
\text { definition }\end{array}$ & $\begin{array}{c}\text { Stevens' } \\
\text { definition }\end{array}$ & Conversion \\
\hline texture size & $G_{s}=3 \cot r$ & $\nabla a=-\left(3 L_{x} L_{y} / H^{2}\right) \cos ^{2} \delta \sin \delta$ & $G_{s}=-\nabla a / a$ \\
\hline texture density & $G_{d}=-3 \cot r$ & $\nabla \rho=3 \rho_{s} H^{2} \sin \delta / \cos ^{4} \delta$ & $G_{d}=-\nabla \rho / \rho$ \\
\hline linear perspective & $G_{L}=\cot r$ & $\nabla w=-\left(L_{x} / H\right) \sin \delta$ & $G_{L}=-\nabla w / w$ \\
\hline texture compression & $G_{C}=\cot r$ & $\nabla e=-\left(L_{y} / L_{x}\right) \sin \delta$ & $G_{C}=-\nabla e / e$ \\
\hline
\end{tabular}

Note: $1 . r$ is the complement angle of $\delta$.

2. Parameters $L_{x}, L_{y}$, and $\rho_{s}$ denote the actual width, height, and density of texture elements, and $\mathrm{H}$ is the height of the eye above the surface.

The surface's geographical slant $(\theta$ in Figure 1.3b) can also be inferred from texture gradient. Unlike optical slant, geographical slant is defined using a world coordinate system as the inclination of a surface with respect to the horizontal plane. Thus it remains constant over changes in posture or eye position. In theory, if the viewing direction is known and optical slant is specified by texture gradient information, 
geographical slant can be computed from a compensatory relation between the viewing direction and optic slant $\left(\theta=r-U=\left(90^{\circ}-\delta\right)-U\right.$ in Figure 1.3c) (Purdy, 1960).

If applied to distance perception, local texture gradient cues are able to specify an egocentric distance on a level ground. Following the computational scheme in Figure 1.1a, the distance is related to local texture gradients by computing $\tan (r)$ from texture gradients. That is, $\mathrm{D}=H^{*}\left(G_{D} / 3\right)=H^{*}\left(-G_{S} / 3\right)=H^{*}\left(-G_{L}\right)=H^{*}\left(-G_{C}\right)$ (Equation 1.1), where $G_{d}, G_{s}, G_{C}$ and $G_{L}$ denote texture gradient of density, size, compression and perspective, respectively (Purdy, 1960).

\section{Slant perception from texture gradients}

Previous studies have confirmed the correspondence between the perceived surface slant and texture gradient information. Consider how optical slant changed with the magnification of a picture. By Purdy's definition of texture gradient, if a picture is magnified by $m$ times, local texture gradients will be reduced by $1 / \mathrm{m}, G_{m}=\frac{1}{m} G$. Accordingly, the slant specified by texture information will accordingly reduce, $\delta_{m}=\arctan \left(\frac{\tan \delta}{m}\right)\left(\right.$ Purdy, 1960; Lumsden 1980). Specifically, a slant of $60^{\circ}$ will look like $49.1^{\circ}$ under 1.5 times magnification. Purdy's (1960) experiments perfectly confirmed this prediction: the slant of $60^{\circ}$ under 1.5 times magnification was perceived exactly same as the slant of $49.1^{\circ}$. The slant of $49.1^{\circ}$ was judged as $41.8^{\circ}$ while the slant of $60^{\circ}$ was reported as $42.5^{\circ}$ under the magnification.

However, the underestimation in surface slant perception should be noted. Although texture information is a powerful cue for surface slant, texture gradient alone cannot produce veridical slant perception. The perceived optical slant from texture 
gradient is generally less than the theoretical value and the perception is biased toward the frontal plane, called 'regression to the frontal plane' by Gibson (for review see Sedgwick, 1986). For example, when the density gradient in a random-dot pattern was used to generate a slant of $40^{\circ}$, the perceived slant was $17.12^{\circ}$ for binocular perception and $9.7^{\circ}$ for monocular perception (Clark et al. 1956).

Frontal regression also happens in the perception of geographical slant. Proffitt et al. reported that subjects exaggerated the inclinations of the hills in the visual awareness. That is, hills always look steeper than they really are (Proffitt et al. 1995; Creem \& Proffitt, 1998; Bhalla \& Proffitt, 1999; Proffitt et al. 2001).

Relative slant change, on the other hand, can be perceived accurately from texture gradient. Knill (1998a-c) systematically investigated how texture information would affect slant discrimination. In his experiments, slant discrimination threshold was measured using a $2 \mathrm{AFC}$ procedure, in which subjects were required to judge which of the two simultaneously presented images seemed to be more slanted. His results reported the discrimination threshold as small as $1.2^{\circ} \sim 2.1^{\circ}$ for the test slant of $70^{\circ}$.

\section{The perception of distance from texture gradients}

The usefulness of texture gradients such as compression and perspective in specifying distance has been empirically verified (Smith, 1958; Newman et al, 1973; Levine \& Rosinski, 1976; Yonas et al., 1986). For example, Levine \& Rosinski (1976) had subjects monocularly view a target resting on a background surface, which could be textured with different patterns: blank, horizontal stripes, or a grid of squares. Compared to the blank background condition, subjects' distance estimation was more accurate on the striped surface where texture compression was present. The judgment was most 
accurate on the grid of squares when linear perspective was available. Yonas et al (1986) examined infants' ability to perceive distances from linear perspective and other texture cues. Their results showed that the visual system is capable to make use of pictorial depth information as early as 7 months of age.

Texture density information, on the other hand, has been proved to be ineffective in human distance perception, although Gibson emphasized "the crucial importance of the density of optical texture" because it can be theoretically applied to distance judgment by the rule of "equal amounts of texture for equal amounts of terrain" (Gibson, 1979). For homogenous texture that is uniformly distributed across a surface, there exists a correspondence between distance and the amount of texture: a particular distance always covers the same amount of texture. Texture thus can be used as a "yardstick" for measuring distances. Gibson suggested that when judging distance, observers "might have been detecting, without knowing it, the amount of texture in a visual angle" (p161162, Gibson, 1979). However, theoretical analysis shows the difficulties in applying texture density information to distance perception: under perceptive projection, changes in texture density may result from either changes in distance or variations in surface orientation (Stevens 1981, 1984). Previous experiments show little texture density effect on distance perception (Wohlwill, 1963, 1965; Newman 1971). For example, Newman (1971) examined the efficacy of the density cue by gradually increasing texture density on the surface from near to far. If the increasing density could affect distance perception, subjects would perceive the mid-point of the surface beyond the actual position. But Newman found no significant difference in the bisection judgments when comparing the density-increasing texture with the evenly-distributed texture. 


\section{Other depth information in the ground texture}

Besides texture gradients, the texture pattern also naturally carries binocular disparity and/or motion perspective information if viewed with two eyes and/or head motion. These cues are effective and crucial for space perception at least at near distances. They can significantly improve the accuracy of the perception of surface slant and distance (Clark et al. 1956; Flock 1964a; Braunstein, 1968).

To sum up, the ground texture provides rich depth information about the geometry of the surface. By integrating all these information, the brain may yield a correct ground surface representation in the perceptual space and thus be able to judge distance accurately.

\subsection{Outline of this study}

Briefly, two issues will be addressed in this dissertation: the perception of angular declination and the representation of the ground surface.

The first issue will be addressed in Chapter 2. The questions to be answered are: Is angular declination an effective cue for judging distance within action space? Is the visual system able to accurately perceive the information?

Chapters 3 7 consider how the ground surface is represented in the visual world. The "what" question will be first examined. Given that texture is a source of information for representing the surface, Chapter 3 will investigate which of multiple texture cues are effective for representing the ground. Note that the relative effectiveness of different texture cues remains a controversial topic. For example, Cutting \& Millard (1984) reported that texture compression was the only cue for judging curved surfaces, while 
linear perspective and some contribution from texture density gradient were used for judging flat surfaces. In contrast, Knill's (1998a-c) ideal observer model of surface slant perception found that the density cue was ineffective for texture-based estimation of surface slant and the compression cue was the dominant source of information for slant judgment although both perspective and compression cues were useful. In Chapter 3, the role of linear perspective and texture compression in representing the ground surface will be examined.

Chapters $4 \sim 7$ consider how a ground surface representation is computed from the texture information. In Chapter 4, the sequential surface integration process (SSIP) hypothesis is proposed to understand the ground representation process. The SSIP hypothesis argues that a global ground surface representation is formed by an integrative process that samples and combines local information across time and space. To assess this, experiments were conducted in virtual (Chapter 5) or real (Chapter 6) environments. In Chapter 7 an alternative hypothesis, the "boundary" theory proposed by Feria et al. (2003), will be evaluated. 


\section{CHAPTER 2}

\section{DIRECTION CONSTANCY IN SPACE PERCEPTION}

This chapter explored the role of the angular declination information in space perception. The first experiment was to examine the influence of angular declination on distance judgment. Experiments $2.2 \& 2.3$ moved on to measure subjects' perception of angular declination. The findings from these experiments show that a target's angular declination is accurately perceived. The prism study in Exp. 2.4 further illustrates that the visual system does assess the information regarding the angular declination for space perception.

\subsection{Exp 2.1. Influence of angular declination information on distance judgment}

Before we move on to measure the perceived angular declination, we want to make sure that angular declination is really used to compute distance by the visual system. The issue has been addressed somewhat by Philbeck \& Loomis (1997). They tested subjects in the dark and the target was placed either at the observer's eye level with a constant angular declination $\left(0^{\circ}\right)$ or on the ground with variable angular declinations at different locations. In the former condition, the perceived distance was nearly constant $(\sim 3.8 \mathrm{~m})$, although the target distance increased from $1.99 \mathrm{~m}$ to $5.0 \mathrm{~m}$. On the other hand, 
the perceived distance did increase somewhat with the target distance in the latter condition. This indicated that angular declination might be a useful cue for distance.

In this experiment, we further examine the question of whether angular declination is an operative cue for distance. The experimental design was similar to Philbeck \& Loomis'. The target was placed at different heights and its angular declination systematically varied: its angular declination kept constant in the target-ateye-level condition but changed most in the target-on-the-ground condition. Accordingly, we expected that subjects' distance perception would be influenced least in the former condition but most in the latter condition by the angular declination information.

In addition, distance judgments were measured by using both the verbal report and the blindfolded walking paradigms. The objective was to compare two methods that would be used in this and following studies.

\subsubsection{Methods}

Observers. Eight paid adults, 6 men and 2 women, participated in the experiment. All subjects had normal or correct-to-normal vision and were naïve about the purpose of the study.

Design. Three independent variables were used in a cross-factor design with 2 measures for each combination of factors. These factors were: (1). the task (verbal report or blindfolded walking); (2). the target's height (on the ground, $0.5 \mathrm{~m}$ above the ground, $0.5 \mathrm{~m}$ below the eye level, or at the eye level); (3). the target's distance $(1.5,2.5,3.75,5.0$, 6.25 and $7.5 \mathrm{~m}$ for blindfolded walking trials; $1.5,2.5,5.0$, and $7.5 \mathrm{~m}$ for verbal report trials). The experiment was blocked by different tasks, but the presentation of the target 
within each block was fully randomized. The testing order of these blocks was balanced through subjects.

Experimental Setting and Stimuli. The experiment was conducted in a carpeted hallway $(3 \times 11 \mathrm{~m})$. After room lights were extinguished, there was no visible reference in the environment.

The target was an internally illuminated Ping-Pong ball (luminance: $0.16 \mathrm{~cd} / \mathrm{m}^{2}$ ) housed in a small box with an adjustable aperture. Before each trial the aperture was scaled to the pre-calibrated size so that the target had a constant visual angle of $0.23^{\circ}$ when seen at the eye height. The target was mounted on a tripod-like stand. On a given trial, it was placed at the preset height and distance.

Procedure. Subjects were tested individually. Their eye heights were measured before the test. After reading the instruction, subjects completed at least five practice trials for each task to familiarize them with the procedure. These trials utilized the same procedure as in the test, but were performed in another hallway so that under no circumstance would subjects find out the experimental setup in advance. During the practice, no error feedback was given.

After that, the subject was led to a waiting room in the experiment hallway with eyes blindfolded. He/She stayed there until the experimenter had set the stimulus. At beginning of each trial, the subject was led to the starting position and informed the task, to give a verbal estimate or to perform blindfolded walking. Then the experimenter turned on the target and the test started. Subjects were asked to stand upright when observing the target. No instrument was used to control their posture and head position. 
For verbal report trials, the subject was asked to estimate the distance to the target and loudly report a numerical estimate in feet and inches, or meters and centimeters. After giving report, the subject returned to the waiting room immediately, and stayed there until the next trial was ready.

For blindfolded walking trials, the subject stood at the starting position, viewed the target, and estimated the distance to it. When ready, he/she first blindfolded his/her eyes and informed the experimenter to remove the target out of the way. Then the subject quickly and decisively walked towards the target and stopped at where he/she thought the target was. The experimenter placed a marker, which was invisible in the dark, on where the subject stood, and led him/her back to the waiting room with eyes still blindfolded. The subject would stay there until the next trial was ready. After the whole experiment was done, the experimenter measured and recorded the walked distances.

\subsubsection{Results}

The data for four target heights, averaged across eight subjects, are shown in Figure 2.1. First of all, all subjects showed the same pattern in both tasks: the perceived space was compressed in depth. They tended to underestimate far distances (6.25 and $7.5 \mathrm{~m})$ and overestimate near distances $(1.5$ and $2.5 \mathrm{~cm})$. Take the blindfolded walking as the example. When the target was on the ground, the mean walked distance was $1.644 \mathrm{~m}$ and $5.639 \mathrm{~m}$ for the target distance of $1.5 \mathrm{~m}$ and $7.5 \mathrm{~m}$, respectively. The perceived distance increased by only $3.995 \mathrm{~m}$ despite that the target moved away by $6 \mathrm{~m}$.

Second, no significant task difference was found in subjects' responses. The mean verbal responses and walked distances were very close to each other while the verbal responses showed relatively larger variations. A three-way repeated measures ANOVA 
(Task $x$ Distance $x$ Height) found no significant main effect of Task $(F(1,7)=0.344$, $\mathrm{p}=0.576$ ) and no significant interactions among Task and other two factors (the interaction of (Task $x$ Distance): $F(3,21)=1.116, p=0.365$; the interaction of (Task $x$ Height): $F(3,21)=1.010, p=0.408$; the interaction of (Task $x$ Distance $x$ Height): $F(9,63)=1.269, p=0.271)$.

Figure 2.2 plots the verbal reports as a function of the walked distances. The mapping between two types of responses is highly positive correlated $\left(r^{2}=0.6424\right.$, $\mathrm{p}<0.001)$ and well fit by a linear function. This suggests that both responses are controlled by a common internal variable, apparently perceived distance, and thus can be equally used as indicators of distance perception. Also, we notice that the slope of the regression is slightly larger than 1 . That is, verbal responses were a little larger than walked distances. The experiments in the next chapter also found overestimation in the verbal report. This might be attributed to different output transforms of perceived distance in two tasks (Foley, 1977).

Most important, subjects' distance perception is significantly influenced by the target's height. As the target was moved toward the observer's eye level, the overestimation/underestimation of near/far distances increased. For example, for the target distance of $1.5 \mathrm{~m}(7.5 \mathrm{~m})$, the mean walked distance was $1.644 \mathrm{~m}(5.639 \mathrm{~m})$ in the target-on-the-ground condition, but increased (reduced) to $3.04 \mathrm{~m}(5.129 \mathrm{~m})$ in the targetat-the-eye-level condition. Compared to the target-on-the-ground condition, the perceived space was compressed almost by half in the target-at-the-eye-level condition $(2.089 \mathrm{~m}$ vs. 3.995m). A two-way repeated measures ANOVA (Distance $\mathrm{x}$ Height) found a significant main effect of Distance $(F(5,35)=92.024, \quad p<0.001$ for blindfolded walking; 
$F(3,21)=21.502, p<0.001$ for verbal estimation) and a significant interaction of (Distance $x$ Height $)(F(15,105)=3.866, p<0.001$ for blindfolded walking; $F(9,63)=2.346, p<0.025$ for verbal report).

Clearly, the results show that the angular declination information can influence the perception of distance.

\subsection{Exp 2.2. Judging the target's angular declination in the lit environment}

Now we move on to investigate whether the visual system is able to accurately perceive the angular declination information in the well-lit environment. Here the perceived angular declination was measured by using a modified blindfolded-walking paradigm.

\subsubsection{Methods}

Subjects. Nine paid naïve subjects were tested. All had normal or correct-tonormal vision.

Design. The cross-factor design was same as that in the previous experiment. Two independent variables were: (1). the distance to the target $(1.5,2.5,3.75,5.0,6.25$, and $7.5 \mathrm{~m}$ ) and (2). the target's height (on the ground, $50 \mathrm{~cm}$ above the ground, or $50 \mathrm{~cm}$ below the subject's eye level). A total of 36 trails were tested. The presentation of these trials was fully randomized.

Experimental Setting and Stimuli. The experiment was conducted in a well-lit carpeted hallway $(3 \mathrm{~m} \times 11 \mathrm{~m})$. The target was a small yellow plastic square $(3 \mathrm{~cm} \times 3 \mathrm{~cm})$. On a given trial, it was placed on the ground at the given distance, or hung down from the arm of a crane-like device on a thin, barely visible string in the target-above-the-ground 
conditions. To minimize the possibility that the observer might use the crane-arm as the reference for judging the target's height, the length of the string and the height of the arm were adjusted independently and the arm was randomly set at different heights for a particular target height. Also, to prevent subjects from using the experimenter's body as the reference in the height judgment, the experimenter stayed outside the observer's field of view all the time.

Procedure. After all light-off experiments were finished, the experimenter turned on the light and began this experiment.

Subjects' distance estimates were measured using a modified blindfolded walking paradigm. On a given trial, the subject stood at the starting position, viewed the target, and estimated its location: the distance to it and its height relative to the ground. When ready, he/she blindfolded his/her eyes first, then quickly and decisively walked towards the target. After reaching the position he/she thought the target was, the subject gestured the perceived height of the target. The experimenter measured the gestured height and the walked distance and then led the subject back to the original position with eyes still blindfolded. Each trail took about 2 min.

\subsubsection{Results}

As shown in Figure 2.3, the estimates of the target's distance and height were quite accurate in the well-lit environment. For distance judgment, the mean walked distances were highly accurate, whether the target was on the ground or not. A two-way repeated measures ANOVA (Distance $\mathrm{x}$ Height) found no significant effect of the target's height on subjects' distance judgment (the main effect of the target's height: $F(2,16)=1.699, p=0.214$; the interaction of (Distance $x$ Height): $F(10,80)=1.223$, 
$\mathrm{p}=0.289$ ). In addition, when the target was above the ground, subjects judged its height very accurately over the distance tested. No systematical errors in height judgments were found $(\mathrm{t}(53)=0.480, \mathrm{p}=0.633$ for the $0.5 \mathrm{~m}$-above-the-ground condition; $\mathrm{t}(53)=1.667$, $\mathrm{p}=0.101$ for the $0.5 \mathrm{~m}$-below-the-eye-height condition).

Figure 2.4(a) plots the perceived location of the target by relating the judged height ( $\mathrm{y}$ axis) to the judged distance ( $\mathrm{x}$ axis). Obviously, the target's location was perceived accurately. Also, if we connect a line from the subject's eyes to the judged target locations, we can obtain the perceived angular declinations with reference to the subject's eye levels. Figure 2.4(b) shows the scatter plot of the relation between the perceived and actual angular declinations, and a strong linear correlation is evident $\left(\mathrm{r}^{2}=0.9742, \mathrm{p}<0.001\right)$. This suggests that the angular declination information is accurately perceived by the visual system in the lit environment.

\subsection{Exp 2.3. Judging the target's angular declination in the dark}

In this experiment we measured the perceived angular declination in the dark.

\subsubsection{Methods}

Observers. The same nine subjects participated in this experiment.

Design. The cross-factor design was the same as Exp 2.2.

Experimental Setting and Stimuli. The experimental settings and the stimuli were same as those in $\operatorname{Exp} 2.1$.

Procedure. The procedure was same as that in the pervious experiment. In addition, it should be emphasized that our subjects performed this experiment before Exp. 2.2. So they had no knowledge about the experimental settings. 


\subsubsection{Results}

Figure 2.5 plots the walked distance as a function of the actual distance to the target. Like Exp. 2.1, the results found a compressed visual space in depth. Observers tended to underestimate large distances and overestimate short distances. Take the target$0.5 \mathrm{~m}$-above-the-ground condition as the example. The mean walked distance was $2.048 \mathrm{~m}$ and $5.225 \mathrm{~m}$ for the target distance of $1.5 \mathrm{~m}$ and $7.5 \mathrm{~m}$, respectively. Moreover, the compression increased as the target was moved closer to the subject's eye level. A twoway repeated measures ANOVA (Distance $\mathrm{x}$ Height) found a significant main effect of the target's height $(F(2,16)=6.213, \mathrm{p}<0.01)$.

Subjects also misperceived the target's height. Generally, the target was seen lower (higher) than its actual location at near (far) distances. When the target was $0.5 \mathrm{~m}$ above the ground, the mean judged height was $15.9 \mathrm{~cm}$ at $1.5 \mathrm{~m}$ and $99.9 \mathrm{~cm}$ at $7.5 \mathrm{~m}$. A two-way repeated measures ANOVA (Distance $\mathrm{x}$ Height) found a significant main effect of Distance $(F(2,16)=40.539, p<0.001)$ and a significant interaction of (Distance $x$ Height $)(\mathrm{F}(10,80)=2.498, \mathrm{p}<0.02)$.

Figure 2.6(a) plots the average perceived location for the three target heights tested. In spite of errors in the distance and height judgment, the figures revealed a consistent pattern: if a projection line was drawn from the observer's eyes to the target, the perceived location, although incorrect, was nearly on the line. To further clarify this pattern, Figure 2.6(b) shows the scatter plot of the relation between the perceived and actual angular declinations. Clearly, the sensed angular declination is very close to the actual value and the correlation analysis reveal a strikingly linear relationship between 
them $\left(r^{2}=0.9315, p<0.001\right)$. That is, the visual system accurately perceives the angular declination even in the dark environment.

Compared to the lit condition, the perception of angular declination in the dark has relatively larger variance. Given that subjects were tested twice at each target location, the difference in their judgments is taken as a rough estimate of the withinsubject variance. Figure 2.7 plots the variance in the perceived angular declinations as a function of the actual angular declinations. In both lit and dark conditions, the variance slightly increased with the angular declination. And the variance in perception was significantly larger in the dark than in the light (paired t-test: $t(107)=7.307, p<0.001$ ).

\subsection{Direction constancy in space perception}

From the above findings, we assert the direction constancy rule for space perception: the visual system can accurately perceive the angular declination of a target in $3 D$ space and accordingly the target's position is perceptually located on the projection line from the eye to it even if the perceived location is erroneous. From a computational standpoint, this rule validates the inverse projection model in human vision (Pizlo, 2001). It puts a constraint on perceptual interpretations of proximal stimuli: like in computer vision, a point in the retinal image cannot be arbitrarily interpreted as any position in $3 \mathrm{D}$ space; all possible solutions are confined to the projection line that passes through the point and the eye's optical center.

\subsection{Exp 2.4. The prism-adaptation experiment}

Here the direction constancy rule was examined in a prism-adaptation study. In the experiment, subjects' judgment of the target's location was measured in three 
conditions. First, a baseline was measured in the dark in the same way as the pervious experiment. After that, the subject was asked to wear a pair of bottom-up prism goggles (20 PD) that would shift the target's angular declination downward by $11.5^{\circ}$ (the prism condition). Due to the downward shift of angular declination, underestimation was expected in the distance and height judgment. Next, the subject was exposed to the lit environment. He or she was asked to walk around in the environment so as to adapt to the optical displacement produced by the prisms. Then subjects were tested in the dark immediately after the removal of the prisms (the aftereffect condition). Knowing that the aftereffect of the prism adaptation would reduce the angular declination, overestimation in the distance and height judgment was expected.

In addition, subjects' perceived eye level was measured to assess shifts in the angular declination due to the prisms or the adaptation aftereffect. According to the direction constancy rule, the eye level shift would quantitatively matched with the angular declination shift that was derived from the judged target locations.

\subsubsection{Methods}

Observers. Seven subjects participated in this experiment. All subjects had normal or correct-to-normal vision.

Design. Subjects' judgments of the target's location were measured in aforementioned conditions using the same cross-factor design as in Exp 2.3. In each condition, a total of 36 trails were tested and the presentation of these trials was fully randomized so that subject had no prior knowledge about the target' location.

Experimental Setting and Stimuli. The experimental settings and the stimuli were same as those in Exp 2.3. 
Procedure. Subjects were tested individually in the dark. The perceived location of the target was measured by using the blindfolded walking paradigm described in Exp. 2.2. The perceived eye level was determined by using a matching paradigm with five measurements. In the dark, the subject stood still with his/her head held by a chin rest. A red light target $\left(0.23^{\circ}\right.$ of visual angle) was placed in front of him/her from a viewing distance of $2.4 \mathrm{~m}$. The subject instructed the experimenter to move the target upward or down until it was judged to be at his or her eye level.

The experiment had three phases. First, the subject's judgments of eye level and the target's location were measured in the dark (the baseline condition). Then the same measure was performed with wearing a pair of bottom-up prism goggles (20 PD) (the prism condition). After that, the subject returned to the lit environment and began the prism adaptation. The subject wore the prisms and walked around in the building for 20 min under the protection of the experimenter. Meanwhile, he or she was asked to perform some subtle tasks like pointing. After the adaptation, the subject was led back to the dark hallway. There his or her eye level and distance/height judgments were measured immediately after the removal of the prisms. In addition, to maintain the prism adaptation during the 36 trials of measuring distance/height judgment, the observer wore the prisms in a well-lit waiting room between trials.

\subsubsection{Results}

Figure 2.8 plots the average perceived target locations in three conditions and lines are drawn to illustrate the changes in perceived angular declination. Consistent with our predictions, the perceived angular declination increased in the prism condition (dash lines) and decreased in the aftereffect condition (dot lines) as compared to the baseline 
condition (solid lines). Correspondingly, subjects underestimated or overestimated the target's distance and height, respectively (Distance judgment in the prism condition: the main effect: $F(1,6)=30.941, p<0.002$; the interaction of (Prism $x$ Distance): $F(3,18)=$ $7.620, p<0.005$; Height judgment in the prism condition: the main effect: $F(1,6)=33.620$, $p<0.002$; the interaction of (Prism $x$ Height): $F(2,12)=18.753, p<0.001$; the interaction of (Prism $x$ Distance): $F(3,18)=39.921, p<0.001$. Distance judgment in the aftereffect condition: the main effect: $F(1,6)=9.309, p<0.025$; Height judgment in the aftereffect condition: the main effect: $F(1,6)=13.058, \mathrm{p}<0.02)$.

Figure 2.9(a) plots the average perceived angular declination as a function of physical angular declination. Compared to the baseline, the perceived angular declination increased in the prism condition by $11.63 \pm 1.37^{\circ}(\mathrm{t}(11)=10.17, \mathrm{p}<0.001)$ and decreased in the after-effect condition by $2.96 \pm 0.44^{\circ}(\mathrm{t}(11)=7.99, \mathrm{p}<0.001)$. More important, the judged eye level also shows the same pattern. It shifted downward in the prism condition by $10.38 \pm 1.43^{\circ}(\mathrm{t}(6)=7.258, \mathrm{p}<0.001)$ and upward in the after-effect condition by $2.82 \pm 0.74^{\circ}(t(6)=3.784, p<0.01)$. Shifts in the perceived eye level quantitatively matched well with those in the perceived angular declination $\left(11.63^{\circ}\right.$ vs. $10.38^{\circ}, t(6)=0.103$, $\mathrm{p}=0.92$ in the prism condition; $2.96^{\circ}$ vs. $2.82^{\circ}, \mathrm{t}(6)=0.796, \mathrm{p}=0.46$ in the aftereffect condition). Especially, in the prism condition shifts in perception $\left(11.63 \pm 1.37^{\circ}\right.$ in the perceived angular declination; $10.38 \pm 1.43^{\circ}$ in the perceived eye level) were very close to $11.5^{\circ}$, the physical shift caused by the prisms. This illustrates that the visual system does assess the information regarding the angular declination for space perception and hence a shift in the target's angular declination is accurately perceived. 


\subsection{Summary}

The above experiments provide direct support for the direction constancy rule. The results clearly demonstrate that angular declination is a strong cue for distance. Even in the cue-reduced dark environment, the angular declination information can be accurately registered by the visual system and a target is always visually located on the projection line from the observer's eyes to it. 


\section{CHAPTER 3}

\section{EFFECTIVENESS OF TEXTURE CUES IN DISTANCE PERCEPTION}

So far, we have established that the visual system can accurately perceive the angular declination of the target and use it as a cue for distance. Veridical perception of action space is thus reducible to constructing a correct representation of the ground in the perceptual world, given that eye height can also be accurately registered (Stoper \& Cohen, 1986, 1991; Sinai et al., 1998). This and next four chapters will address some issues on defining the ground surface representation: Provided that texture on the ground is a significant source of information, what textural cues are actually effective for representing the surface and accordingly supplying veridical distance perception? And how does the brain build an accurate ground representation from texture information?

The current study aims to answer the "what" question. The first experiment was to establish the reliability of the verbal estimation paradigm for measuring subjects' distance perception. The following six experiments investigated the relative effectiveness of different texture cues by using the cue-conflicting paradigm. The presence of different texture cues on the ground was carefully controlled, either in concordance with or in conflict with other cues, and the effectiveness of these cues was determined by their power of influencing subjects' distance estimation. Experiments 3.2 3.5 compared the 
effectiveness of linear perspective and texture compression cue. The findings from these experiments reveal that linear perspective is a very effectual cue for distance but texture compression not. The power of linear perspective on distance judgment was further examined in the experiment 3.6 by using the blindfolded throwing task. At last, we suggested a model of how linear perspective could affect the representation of the ground and hence distance perception, which was assessed in Exp. 3.7.

\subsection{Exp 3.1. Distance judgment with a continuous ground texture}

The experiment measured subjects' distance judgment in the dark using the verbal estimation paradigm. The purpose was twofold: to demonstrate the reliability of the measurement method and to demonstrate the significance of the ground texture in distance perception.

\subsubsection{Methods}

Observers. Eight paid naïve subjects with normal or correct-to-normal vision participated in the experiment.

Design. Five distances were tested $(1.5,2.5,3.75,5.0$, and $6.25 \mathrm{~m})$ with two measures for each. The presentation of the target distance was fully randomized. Also, a baseline measure was performed in the well-lit environment after all experiments in the dark.

Experimental Setting and Stimuli. The experiment was conducted in a dark carpeted hallway $(3 \times 11 \mathrm{~m})$. After room lights were extinguished, there was no visible reference in the environment, except for the texture pattern on the ground, as shown in 
Figure 3.1(a). In addition, to help the observer scale the perceived distance, the standard length of 1 meter or 1 yard was denoted by two markers placed before the observer's feet.

The target was an internally illuminated Ping-Pong ball housed in a small box with an adjustable aperture. The aperture was scaled to the pre-calibrated size before each trial so that the stimuli had a constant visual angle of $0.23^{\circ}$ when seen at the eye height. The luminance of the stimuli was $0.16 \mathrm{~cd} / \mathrm{m}^{2}$.

Procedure. Subjects were tested individually. After reading the instruction, subjects completed at least five practice trials to familiarize them with the procedure. Their distance estimates were measured using the verbal report protocol. At beginning of each trial, the subject was led to the starting point and then the experimenter illuminated the target. The subject was asked to judge the distance from his or her feet to the target, and loudly report a numerical estimate in feet and inches, or meters and centimeters. After giving report, the subject was led to a waiting room immediately and waited there until the next trial was ready. In the experiment no error feedback was provided.

\subsubsection{Results}

In the section 2.1 we have shown that verbal report is an indicator for perceived distance. Here we examine the Test-Retest reliability of the verbal report paradigm. Figure 3.2 plots pairs of reports of the target distance against each other and demonstrates good repeatability in subject's responses. Two measurements were comparable and there was no systematical change from the first report to the second. Intra-class correlation coefficients between two judgments were calculated for all distances tested. All coefficients reached the significance level at $\mathrm{p}<0.05\left(r_{\min }=0.7294\right.$ for the target distance 
of $1.5 \mathrm{~m}$ in the texture-in-the-dark condition). The analysis thus demonstrates that the verbal report paradigm is a reliable measure of perceived distance.

The mean reported distances, averaged across 8 subjects, are given in Figure 3.1(b). In contrast to distance underestimation in the totally dark (the filled squares in the figure, data from Exp 2.1), the perception of distance became reasonably accurate when the texture made the ground visible. Subjects' performance was nearly identical to that in the light. Their verbal estimation of distance was linear correlated with the actual distance $\left(\mathrm{R}^{2}=0.762, \mathrm{p}<0.001\right.$ in the light condition and $\mathrm{R}^{2}=0.808, \mathrm{p}<0.001$ in the textured ground condition) and showed no significant difference between the texture-in-the-dark condition and the in-the-light condition $(F(1,7)=1.190, p=0.104 ; F(4,28)=2.258$, $\mathrm{p}=0.088$ ). The results indicate that the ground texture is a reliable source of information for judging distance accurately.

\subsection{Exp 3.2. The effect of false linear perspective on distance perception}

Knowing that texture is a significant source of information for representing the ground and judging distance, the current and following three experiments aim to find out what texture information is in fact effective. Linear perspective was first examined in this experiment by using the cue-conflicting paradigm. The rationale is that, when conflicting information comes from two sources, judgments may be based on one stronger cue, with the other cue being suppressed. Here false line perspective was introduced by presenting converging lines on the ground. Accordingly, distance overestimation was expected if the false perspective information was effective and dominant. 
Another question to be addressed is that which part of the ground holds more significant information for representing the surface, the area about the observer or the area surrounding the target. The experiment addressed this by texturing a part of the ground $(1.2 \times 3 \mathrm{~m})$ that was in front of the observer's feet or $4 \mathrm{~m}$ away from the observer. The relative effectiveness of depth cues at different locations was compared by the pattern's influence on distance perception.

\subsubsection{Methods}

Observers. Twelve subjects were tested and four of them also participated in the previous experiment. They have normal or correct-to-normal vision. All of them were naïve about the purpose of the present study.

Design. Three independent variables were used in a cross-factor design with 2 measures for each combination of factors. These factors were: (1). cue validity (correct or false linear perspective), (2). the location of texture pattern ( $1 \mathrm{~m}$ or $4 \mathrm{~m}$ away from the observer), and (3). the target's distance from the observer (1.5, 2.5, 3.75, 5.0, and $6.25 \mathrm{~m})$. A total of 40 trails were tested. The presentation of these trials was blocked by the combinations of different texture locations and cue validity, but fully randomized within each block.

Procedure. The procedure was the same as in the previous experiment.

\subsubsection{Results}

As shown in Figure 3.3(b), subjects showed different response patterns at different distances. When the target was near $(1.5 \mathrm{~m}$ and $2.5 \mathrm{~m})$, subjects' distance perception was little affected by linear perspective information. Their estimates of distance in the dark were comparable to that in the lit environment regardless of the 
presence of correct or false linear perspective. This suggests that near depth cues such as oculomotor cues, rather than texture cues, dominate within this range.

As the target distance increased, subjects' judgments revealed a shift toward reliance on texture cues and the effect of false linear perspective became significant in action space. Subjects reported a larger distance to the target when the converging lines of fluorescent dots were present on the ground. For example, the distance of $6.25 \mathrm{~m}$ was overestimated by $24.3 \%(8.81 \mathrm{~m}$ in the correct linear perspective condition vs. $10.95 \mathrm{~m}$ in the false linear perspective condition) in the texture-at-near-location conditions. The three-way repeated-measures ANOVA (Cue validity $\mathrm{x}$ Texture location $\mathrm{x}$ Distance) found a significant main effect of linear perspective $(F(1,11)=19.280, p=0.001)$ and a significant interaction of (Line perspective $x$ Distances $)(F(4,44)=6.918, p<0.001)$.

For the question of which part of the ground holds more significant information for representing the surface, we found a significant role of the near ground area in space perception. When false line perspective was presented at the near location, it yielded larger errors in subjects' judgments as compared to the texture-at-far-location condition. Take the target distance of $6.25 \mathrm{~m}$ as the example. The overestimation due to false linear perspective was $24.3 \%$ in the texture-at-near-location condition and $10.3 \%$ in the textureat-far-location condition, respectively. The correct linear perspective also showed a similar effect. For example, with correct linear perspective, the target distance of $6.25 \mathrm{~m}$ was reported as $8.81 \mathrm{~m}$ and $7.68 \mathrm{~m}$ in the texture-at-near-location condition and the texture-at-far-location condition, respectively. The ANOVA found a significant main effect of texture location $(F(1,11)=35.658, p<0.001)$ and also a significant interaction of (Texture location $x$ Distances) $(F(4,44)=24.267, \mathrm{p}<0.001)$. 
In sum, the results reveal that line perspective is a strong cue for distance, within action space which dominates when it is in conflict with other cues. In addition, the near ground area is important for space perception. The results show that when the ground about the observer was textured, the texture information yielded larger estimates in distance judgment and larger errors in the presence of false linear perspective, as compared to the same texture pattern placed at a far location ( $4 \mathrm{~m}$ from the observer).

\subsection{Exp 3.3. Relative ineffectiveness of texture compression cue}

This experiment was to examine the effectiveness of texture compression cue. Consider the texture pattern in the last experiment. Both linear perspective and texture compression cues were present. For example, since the depth interval between two rows of dots was constant in the false-linear-perspective texture, this regularity provided a valid compression cue that disagreed with false linear perspective. Thus it is possible that both cues are effective and the visual system, when estimating distance, averages their effects with different weights. That is, the visual system might use compression cue to correct distance overestimation from false linear perspective somewhat. To test this possibility, we examined subjects' distance perception when the texture compression cue was removed by texturing the ground with solid fluorescent lines, instead of the grid of dots (Figure 3.4(a)). Thus, if the compression cue is effective and plays a role in distance perception, difference in distance judgments should be expected between the with- and without- compression-cue conditions.

\subsubsection{Methods}

Observers. Eight naïve subjects were tested and two of them participated in the previous experiment. All have normal or correct-to-normal vision. 
Design. The cross-factor design was similar to the previous experiment. The difference was that the location of texture pattern was fixed and the texture-location factor was replaced by the presence-of-compression-cue factor. Three factors were (1). the presence of texture compression cue (with or without valid texture compression cue), (2). the validity of linear perspective (correct or false linear perspective), and (3). the target's distance $(1.5,2.5,3.75,5.0$, and $6.25 \mathrm{~m})$.

Procedure. Subjects' distance perception was measured using the same verbal estimation paradigm as in the previous experiment.

\subsubsection{Results}

First, the experiment replicated the finding that false linear perspective significantly influenced distance perception. Compare the left and right figures in Figure 3.4(b). False linear perspective produced distance overestimation despite of the existence of valid texture compression cue. The three-way repeated-measures ANOVA (Compression-cue $\mathrm{x}$ Linear-perspective $\mathrm{x}$ Distance) found a significant main effect of linear perspective $(F(1,7)=12.927, p<0.01)$ and a significant interaction of (Line perspective $x$ Distances $)(F(4,28)=4.689, \mathrm{p}<0.005)$.

Second, no significant difference was found when comparing the with- and without- compression cue conditions. Subject's distance estimates were nearly identical (the main effect of the compression cue: $F(1,7)=0.394, p=0.550$; the interaction of (Compression cue $\mathrm{x}$ Distance): $\mathrm{F}(4,28)=0.699, \mathrm{p}=0.599$; the interaction of (Compression cue $x$ Linear perspective): $F(1,7)=3.403, p=0.108$; the interaction of (Compression cue $x$ Linear perspective $\mathrm{x}$ Distance): $\mathrm{F}(4,28)=1.383, \mathrm{p}=0.265)$. The results indicate that compression is a relative ineffective cue as compared to linear perspective. When two 
cues were in conflict, the visual system could not use the correct compression cue to improve distance judgment and the false linear perspective cue dominated performance.

\subsection{Exp 3.4. Little to no effect of false compression cue on distance perception}

Here the effectiveness of the compression cue was further examined. The question was: would the visual system be influenced by a false texture compression cue if only this cue was present? In the experiment lines were placed on the ground with smaller intervals in depth as distance increased (Figure 3.5(a)). This theoretically would cause distance overestimation like false linear perspective in Exp 3.2. In addition, linear perspective was eliminated by randomly shifting lines left or right. Overestimation was thus expected in subjects' report of distance if the compression cue was effective.

\subsubsection{Methods}

Observers. Eight new naïve subjects with normal or correct-to-normal vision were tested.

Design. The cross-factor design had three factors: (1). the validity of compression cue (with or without valid texture compression cue), (2). different texture elements (solid lines or lines of dots), and (3). the target's distance $(1.5,2.5,3.75,5.0$, and $6.25 \mathrm{~m})$.

Procedure. The procedure was the same as in the previous experiments.

\subsubsection{Results}

No significant effects of false compression cue were found. Subjects' estimates of distance were almost identical with normal or false compression cue, as shown in Figure 3.5(b). The three-way repeated-measures ANOVA (Compression-cue $x$ Texture elements $\mathrm{x}$ Distance) found no significant main effect or interactions of compression cue (main 
effect: $F(1,7)=0.035, \quad p=0.857$; the interaction of (Compression $x$ Distances): $\mathrm{F}(4,28)=1.229, \mathrm{p}=0.321$; the interaction of (Compression $\mathrm{x}$ Texture elements): $F(1,7)=0.115, p=0.744$; the interaction of (Compression $x$ Texture elements $x$ Distance): $F(4,28)=0.233, p=0.918)$

So far, converging evidence from the above experiments has undoubtedly established that when judging absolute distance on a level ground, the most important cue in the ground texture is linear perspective and texture compression cue has little to no contribution. When these texture cues are in disagreement, our results show that linear perspective dominates perception.

\subsection{Exp 3.5. High efficiency of linear perspective}

In this experiment we further demonstrate the high efficiency of linear perspective on distance perception. Since linear perspective is delineated by the projection of two parallel lines, the minimal texture to produce linear perspective, in theory, is four dots, every two of which define a line. Here normal or false linear perspective was presented by arranging four fluorescent dots as a rectangle or a trapezoid (Figure 3.6(a)). Similar to Exp 3.2, we predicted distance overestimation if false linear perspective in the texture pattern, although weak, had effects on subjects' distance judgments. In addition, the location effect was examined here by setting the texture pattern at two different locations, $1 \mathrm{~m}$ away from the observer or $2.5 \mathrm{~m}$ away from the observer.

\subsubsection{Methods}

Observers. Eight new naïve subjects with normal or correct-to-normal vision were tested. 
Design. The cross-factor design was the same as Exp 3.2 except that the texture location factor took two different levels: the texture pattern was $1.0 \mathrm{~m}$ or $2.5 \mathrm{~m}$ away from the observer.

Procedure. The procedure was the same as in the previous experiments.

\subsubsection{Results}

First, although four dots only provided the minimal linear perspective information, significant distance overestimation was produced by the trapezoid on the ground, especially in the texture-at-near-location conditions. The three-way repeatedmeasures ANOVA (Cue validity $\mathrm{x}$ Texture location $\mathrm{x}$ Distance) found a significant main effect of linear perspective $(F(1,7)=12.751, p<0.01)$ and a significant interaction of (Line perspective $x$ Distances $)(F(4,28)=5.083, p<0.005)$.

Moreover, the amount of overestimation due to false linear perspective was similar to that in Exp 3.2, in which robust linear perspective information was displayed using a $3 \times 4$ array of dots. Take the target distance of $6.25 \mathrm{~m}$ as the example. In the falselinear-perspective-at-near-location conditions, it was overestimated by $24.3 \%$ in Exp. 3.2 and by $27.1 \%$ in the current experiment $(7.50 \mathrm{~m}$ in the correct perspective condition vs. $9.53 \mathrm{~m}$ in the false perspective condition). Obviously, the high efficacy of linear perspective in distance perception is substantiated by the fact that the minimal linear perspective information can change the subjects' performance.

As to the texture-location effect on distance judgment, like in Exp. 3.2, the ground texture was found more effective at the near location than at the far location. As shown in Figure 3.6(b), the distance was perceived larger when the textured ground area was closer to the observer. The ANOVA found a significant main effect of the location of the texture 
pattern $(F(1,7)=8.549, p<0.025)$ and a significant interaction of (Texture location $x$ Distances $)(F(4,28)=3.235, \mathrm{p}<0.05)$.

\subsection{The texture location effect reexamined}

Figure 3.7 plots data from experiments $3.2,3.3 \& 3.5$ together to show how distance judgment is affected by the location of the texture pattern. Clearly, the tendency exists that the closer the texture pattern locates, the larger influence it has on distance perception. This hints that distance is not judged solely using local information about the target. Instead, the near ground information is noteworthy for distance judgment.

The mechanism underlying the texture location effect remains unclear. The effect might result from the difference between the extrapolative and interpolative process. In the near texture condition, the ground representation may be built by an extrapolative progress that initiates from the area about the observer and eventually extends to the empty ground area. On the other hand, an interpolative progress operates for representing the ground in the far texture condition. In the next chapter, the question of how the ground texture is used to judge distance will be addressed.

\subsection{Exp 3.6. The false perspective effect examined using blindfolded throwing}

This control experiment was to negate the possibility that distance overestimation caused by false linear perspective resulted from an exaggerated output transform in the verbal estimation. That is, the internal space representation and the perceived distance might be influenced little by texture cues but false linear perspective might change the output transform function, say, the scaling factor reduced when reporting a numerical 
value of the perception. To test this, subjects' distance perception was examined by using a perception-directed action task: the subject was asked to throw a sandbag to the remembered location of the target with eye closed. Obviously, if the false linear perspective effect were owing to a task-specific change in the verbal estimation function, the throwing would be little influenced by false texture cues.

\subsubsection{Methods}

Observers. Six male athlete-like subjects were tested. According to their selfreport, all were active participants in basketball or volleyball games. They were naive about the purpose of the study and all have normal or correct-to-normal vision.

Design. A baseline was first measured in the light and six distances $(2.5,3.75,5$, $6.25,7.5$, and $9 \mathrm{~m})$ were tested with two repeated measures. After that, subjects were tested in the dark using the same procedure. Three target distances $(3.75,5$, and $6.25 \mathrm{~m})$ were examined with correct or false linear perspective information on the ground.

Procedure. Subjects were tested individually in the experiment with three phases: warming-up, the baseline, and the test in the dark. During the warming-up phase, the subject practiced the blindfolded throwing task. He was asked to remember the experimenter's location and then put on the blindfold and throw a sandbag to the experimenter. With error feedback, after a few throws subjects could perform the task accurately. The warming-up phase took about 15 minutes.

The baseline testing was conducted in a well-lit hallway. At beginning of each trial, the subject was blindfolded until the experimenter had set the target, a red hockey pack (3" in diameter \& 1" in height), at the appropriate distance. The subject then took off the blindfold and examined the distance to the target. When ready, he put on the 
blindfold using his free hand and threw the sandbag to where he thought the target was. The experimenter recorded the sandbag's first landing point and measured the distance of the throw while the subject was still kept blindfolded. No error feedback was provided in the experiment.

The test in the dark was conducted in another hallway, about which subjects had no prior knowledge. The procedure was similar to that in the light. On a given trail, the subject stayed in a waiting room until the experimenter had set the stimulus outside. Then he was led to the original point and the target, a self-illuminated Ping-Pong ball, was then illuminated. The instruction was to judge and remember where the target was and throw the sandbag to that place. When ready, the subject put on the blindfold and notified the experimenter before the throw. The latter then turned on a flashlight and the throw was traced. The sandbag's first landing point was marked and measured. No error feedback was provided in the experiment.

\subsubsection{Results}

As shown in Figure 3.8 (b), the results, averaged across six subjects, showed over-throwing in the presence of false linear perspective. For example, the target distance of $6.25 \mathrm{~m}$ was overestimated by $15.4 \%(6.83 \mathrm{~m}$ in the correct perspective condition vs. $7.88 \mathrm{~m}$ in the false perspective condition). The two-way repeated-measures ANOVA (Cue validity $x$ Distance) found a significant main effect of linear perspective $(F(1,5)=65.099$, $\mathrm{p}<0.001)$.

Clearly, it is implausible that the false perspective effect on distance judgment could be explained by a task-specific change in the verbal estimation, because the same response pattern was found in the blindfolded-throwing task. As an alternative, the same 
response pattern from different tasks indicates that the perceptual space is more likely to be changed by false texture cues on the ground.

\subsection{Exp 3.7. Eye height shift in the presence of false linear perspective}

In this experiment, we investigated how linear perspective affected the representation of the ground and hence distance perception. Since distance is judged on the ground surface, distance overestimation caused by false linear perspective may be explained by an erroneous ground surface representation. Note that the converging linear perspective is related to a descending slope, as shown in Figure 3.9. Thus, when the ground is misperceived as being slanted downward by $\varphi$, distance will be correspondingly overestimated by $d^{\prime}=d^{*} \sin (\alpha) / \sin (\alpha-\varphi)$ (Equation 3.1), where $d$ and $d^{\prime}$ are the actual and perceived distance respectively and $\alpha$ specifies the angular declination of the target.

Here the experiment quantitatively examined the above explanation. The experiment examined subjects' perception of distance and the ground surface in the correct and false linear perspective conditions. Distance perception was directly measured using the verbal estimation paradigm. The ground surface representation was indirectly obtained from the perceived eye level. The paradigm for measuring subject's eye level is shown in Figure 3.10(a). Given that the eye level can be judged relative to the ground plane, false linear perspective on the ground will change both the perceived eye level and the ground representation in the similar way. Here we predicted that both would be shifted downward by the false linear perspective. 


\subsubsection{Methods}

Observers. Six new naïve subjects were tested. All have normal or correct-tonormal vision.

Design. Subjects' distance perception was examined at five locations $(1.5,2.5$, $3.75,5.0$, and $6.25 \mathrm{~m}$ ) in the correct and false linear perspective conditions. Their perceived eye heights were also measured in the same conditions at four locations ( 2.5 , $3.75,5.0$, and $6.25 \mathrm{~m})$. The testing order of these locations was fully randomized.

Procedure. Subject's physical eye heights were measured before the test. On a given trail for measuring the perceived eye level in the dark, two stimuli were presented. The reference stimulus was placed on the ground at the approximate distance and the target stimulus at the same distance but in the air. The subject was asked to keep his/her eye fixation on the reference stimulus so that they were encouraged to use the ground as the reference plane for judging the eye level. The task was to judge if the target stimulus was higher or lower than the subject's physical eye level and accordingly instruct the experimenter to move the target stimulus downward or upward until it appeared at the eye level. At each distance, the perceived eye level was measured with four repeats.

Subjects' distance judgments were measured using the verbal estimation paradigm as in the previous experiments.

\subsubsection{Results}

Consider the judged eye level first. Consistent with previous studies, the judgments of eye level in the cue-reduced dark environment were slightly lower than the physical level. For example, the mean and standard deviation for eye-level judgments in the correct perspective condition was $4.38 \pm 1.99^{\circ}$, which was similar to $2.79^{\circ} \pm 1.72^{\circ}$ 
reported by Stoper \& Cohen (1986). More important, the judged eye level was significantly lower in the false linear perspective condition $\left(6.90 \pm 2.40^{\circ}\right)$ as compared to the correct perspective condition. The two-way repeated-measures ANOVA (Cue validity $x$ Distance) found a significant main effect of false linear perspective $(F(1,5)=204.035$, $\mathrm{p}<0.001)$.

For distance perception, like previous experiments, subjects significantly overestimated distance in the false linear perspective condition. The two-way repeatedmeasures ANOVA (Cue validity $\mathrm{x}$ Distance) found a significant main effect of false linear perspective $(F(1,5)=13.746, p<0.02)$ and a significant interaction of (Texture location $x$ Distances $)(F(4,20)=9.346, p<0.001)$.

Results from both tasks were quantitatively related using the Equation 3.1. The open symbols in the left column of Figure 3.10(b) showed the misperceived slant of the ground derived from means of judged distance. Clearly, the values matched well with the shift in eye-level judgments. Similarly, if distance perception was estimated from eyelevel judgments, the curves in right column of Figure 3.10(b) matched with subjects' distance judgments. Therefore, the results provide evidence for the proposed explanation that false linear perspective causes the misrepresentation of the ground surface and thus yields overestimation in distance judgments.

\subsection{Summary}

In summary, this study shows that distance can be judged accurately from the ground texture information (Exp. 3.1) and linear perspective is identified as a powerful cue for judging distance on a planar ground but texture compression is little effective 
(Experiments 3.2 3.6). When two cues are in conflict, our data indicate that linear perspective dominates perception and even false information from a minimal linear perspective pattern is able to significantly change subjects' performance. These findings are consistent with Cutting \& Millard's (1984) results that linear perspective is the most important cue for judging flat surfaces. Distance overestimation caused by false linear perspective is thus thought as a consequence of a misrepresented ground plane. And this idea has been justified in Experiment 3.7 where the relationship between subjects' distance perception and their perceived ground surface was verified.

Another finding is the significant role of the near ground area in space perception. For subjects' distance perception, the closer the texture pattern locates, the more effective it is. That is, the near ground area is more influential than distant ground areas in space perception. In the next chapter, the critical role of the near ground area and how the ground texture information is used to judge distance will be further discussed. 


\section{CHAPTER 4}

\section{SEQUENTIAL SURFACE INTEGRATION PROCESS HYPOTHESIS}

The experiments in the last chapter have established that texture on the ground is a significant source of information for representing the surface. But so far the "how" question has not been addressed: How can the ground surface be visually represented by using texture information and other depth cues? Particularly, the texture-location effect suggests that distance judgment can be affected by the information that is far from the target. It thus raises the questions: Why does the visual system need to use the seemingly irrelevant information and how is it used?

Here we argue that veridical space perception is based on the representation of an extended ground surface that stretches from the observer to the target and the visual system needs to take in all information on the ground including that is far from the target to yield a global ground surface representation. A Sequential Surface Integration Process (SSIP) hypothesis is proposed to explain how to form a global surface representation. The SSIP hypothesis claims that the process of representing the surface combines depth information across time and space and uses the reliable near depth cues to calibrate the overall representation. 


\subsection{Space perception: an integrative process}

According to Marr's computational theory (1982), early visual processing creates a viewer-centered representation of the surface layout $\left(2 \frac{1}{2}-\mathrm{D}\right.$ sketch), which integrates information from different parts of the image or different cues at the same location like stereopsis, shading, texture. Thus it is expected that when constructing an overall representation of the ground surface and hence judging distances on it, the visual system relies on the integration of depth information across a scale of space and time.

Information integration allows space to be perceived over a larger range than is possible with any single cue alone. Most depth cues have limited effective ranges. For example, the effectiveness of oculomotor cues is confined to a range up to only about $3 \mathrm{~m}$; binocular disparity and motion perspective are ineffective beyond about $30 \mathrm{~m}$. Therefore the visual system cannot develop a global representation of an extended ground surface by using just one cue. Instead, previous studies have shown that the operative depth cues shift with increasing distance from some sources of information to others (Nagata, 1991; Cutting \& Vishton, 1995).

In particular, the integrative process can resolve the ambiguity form any single cue. According to Gogel's (1977) classification, absolute depth cues such as oculomotor cues are able to determine the distance, but information from relative depth cues such as binocular disparity or motion perspective is ambiguous unless the location of the fixation point is known. Take binocular disparity as the example. When the fixation point is near, the meaning of a certain binocular disparity is very different from when the fixation point is far. Thus, to resolve the ambiguity, the visual system must use absolute depth cues to calibrate information in relative depth cues. Essentially, the calibration is a progressive 
process that starts from a near location where the fixation point can be precisely determined by using oculomotor cues and then gradually extrapolates to distant locations. By this way, information from different cues is accumulated across space and veridical space perception is possible.

Experimental evidence supports the hypothesis of space-perception-frominformation-integration. Our experiments reported in the last chapter and Sinai et al's (1998) experiments show that local depth information only is insufficient for veridical distance perception. For example, Sinai et al. found that a continuous texture on the ground is critical for accurate distance judgment. In their experiments, subjects judged distance in three conditions: a target was seen on a uniformly-textured, continuous ground (the control condition), on a differently-textured ground (the texture-discontinuity condition), or across a gap in the ground (the gap condition). Distances were underestimated in the texture-discontinuity condition but overestimated in the gap condition. Only when a continuous ground texture was accessible, the observer judged distances accurately. Note that the local depth information about the target was identical in all three conditions. The finding suggests that distance cannot be estimated accurately from the local depth information about the target. Alternatively, a global representation of the ground is decisive and the visual system needs to take in all information on the ground including that is far from the target to yield a global ground surface representation.

Another evidence was reported by Yarbrough et al. (2002) and He et al. (2004). They asked subjects to judge the distance to the target that was placed behind a wall. Owing to the partial occlusion of the ground, the distance was significantly underestimated. However, an accurate space representation could be obtained through 
information selection, say, using a roundabout strategy in the occlusion condition. If the subject was asked to make a detour (to walk to a turning point first, and then walk to the target behind the wall), they could reach the target with reasonable accuracy. This reveals that veridical space perception can be achieved if the entire ground surface is sampled, even if having to use a roundabout strategy. Thus, it can be concluded that veridical space/distance perception on the ground relies on a process that integrates local ground information across time and space to form an overall representation.

\subsection{The Sequential Surface Integration Process (SSIP) hypothesis}

On the basis of the above results and analysis, we believe that the representation of the ground in visual space and hence the ground-based space perception relies on a process that integrates local ground information to form a global surface representation. Here we propose a Sequential Surface Integration Process hypothesis (SSIP, see Figure 4.1) to explain how the ground surface can be correctly represented.

The SSIP hypothesis essentially extends the original idea of Gibson \& Cornsweet (1952). Gibson and Cornsweet proposed that the global surface layout of the ground could be computed by integrating local texture information seen in successive fixations:

"Take as an example the visual experience of a man standing on a level desert plain and looking about. This example is particularly significant since it is kind of minimum perception for any sort of spatial behavior. What he sees is a level ground extending to the horizon with himself standing on it. No impression of slant seems 
to be evident. But this perception of the earth is almost certainly a product of the integration of successive eye-fixations. Ordinarily the man is unaware of his saccadic eye movements, but if he attempts to introspect, he may discover that every fixation yields a clear momentary impression of a small segment of the ground which does have a kind of slant. As he looks downward toward his feet the slant approaches zero, as he looks upward the slant increase, as the center of clear vision approaches the horizon the slant becomes maximal, and at the horizon it self the land ceases to be a surface and becomes an edge" (p11-12, Gibson \& Cornsweet, 1952).

The idea is that some global properties of the ground surface such as levelness are obtained by integrating information seen in successive fixations. For each piece of the ground seen in fixations, its geographical slant could be computed from a compensatory relation between the viewing direction and the optical slant specified by texture gradient (illustrated in Figure 1.3(c)). A broad picture of the whole surface is then obtained by bringing these pieces together.

Following this approach, we propose the SSIP hypothesis. Given that texture information only is insufficient for the visual system to construct an accurate surface representation (Clark et al. 1956; Braunstein, 1968; Proffitt et al. 1995), the SSIP process initiates the computation from the near space where rich depth cues are available, and gradually extends it to distant areas. More precisely, within near space $(<2 \sim 3 \mathrm{~m})$, the 
visual system refers the body as the reference and uses depth cues such as oculomotor cues and binocular disparity to build an accurate representation for the ground area about the observer's feet $\left(S_{1}\right.$ in Figure 4.1$)$. Then the visual system extrapolates the computation to the neighboring area, $S_{2}$, and adds it in the surface representation. After $S_{2}$ is represented, the SSIP process further spreads to $S_{3}$ and eventually integrates all local areas into an overall surface representation.

Essentially, the idea underlying the SSIP hypothesis is that, the near surface area is correctly perceived and always adopted as the reference to compute distant areas so that eventually the whole surface is represented accurately. Consider the $S_{2}$ in Figure 4.2. Albeit depth cues such as oculomotor, disparity, or motion perspective may be ineffective at far distances, the representation of $S_{2}$ can be computed by tracing continuous textural variations along the surface. The integrative process computes the orientation of $S_{2}$ from its relative slant with respect to $S_{1}$, rather than deducing surface slant directly from local depth information. Because the perception of relative slant change is accurate (Stevens, 1983; Knill, 1998a-c), the orientation of $S_{2}$ is accurately perceived if $S_{1}$ has been correctly registered. Similarly, the location of $\mathrm{S}_{2}$ is also uniquely determined by the SSIP process. Although there exist numerous projectively equivalent configurations for $\mathrm{S}_{2}$ like $S_{2}^{\prime}$ in Figure 4.2(b), the ambiguity can be resolved from the surface continuity that is seen from smooth changes in the ground texture. To sum up, rather than directly perceiving surface layout from local depth information, the SSIP process, like a jigsaw puzzle player, constructs a surface representation by progressively adding new surface pieces into a representation that has been accurately perceived and thus resolves the ambiguity in perception. 


\subsection{Predictions from the SSIP hypothesis}

When applied to the perception of distance on the ground, following propositions are suggested by the SSIP hypothesis.

\section{The critical role of the near ground area}

According to the SSIP hypothesis, a global representation of the ground is constructed by first forming an initial representation and then progressively incorporating new surface pieces into it. Thus an accurate initial representation is crucial for assuring the correctness of the process. To achieve this, the SSIP hypothesis suggests that reliable depth cues in near space play a vital role. That is, the ground area about the observer is accurately represented first and then used as the "anchoring" framework for representing the next surface area.

Without near depth information, the SSIP hypothesis predicts errors in representing the ground surface because the representation is obtained mainly from pictorial cues. Take the perception of surface slant as the example. Although texture gradients are powerful cues for surface slant (Purdy, 1960; Stevens, 1979), they alone cannot produce veridical slant perception and slant judgment will be influenced by projective distortions and the "frontal shift" tendency (for review see Sedgwick, 1986). That is, a flat ground will be misperceived as an ascending slope ( $\mathrm{S}^{\prime}$ 'in Figure 4.3): it gradually elevates in the visual field and ultimately disappears at the eye height as it recedes away from the observer. Accordingly, egocentric distance will be underestimated (Figure 4.3a). For a perceived slant of $\delta$, the perception of distance (d') is given by $d^{\prime}=d^{*} \sin (\alpha) / \sin (\alpha+\delta)$ (Equation 4.1), where $\mathrm{d}$ is the actual distance and $\alpha$ specifies the angular declination of the target with respect to the eye level. In addition, if a L-shape 
is set on the ground, its appearance, measured by the aspect ratio ( $r=d e p t h / w i d t h)$, will be compressed on S' (Figure 4.3b). The perceptual-to-physical ratio of the L-shape's aspect ratio ( $R=$ perceived aspect ratio/physical aspect ratio) can be related to the angular declination and the misperceived ground surface by $R=\sin (\alpha-\Delta \alpha) / \sin (\alpha-\Delta \alpha+\delta)$ (Equation 4.2).

\section{The directional asymmetry in the integrative process}

The SSIP hypothesis also predicts a directional asymmetry in the integrative process of representing the ground surface because the process always uses the perceived surface portion as the reference framework for representing the next surface region. Consider an integration process in the near-to-far direction. When the process is executed in this way, a correct initial representation can be constructed from reliable depth cues in near space and the correctness of the following computation is thus ensured. So the nearto-far integration may yield veridical space perception. On the other hand, if the computation starts from a far place where weak depth cues fail to yield a correct initial representation, the following computation and the final result will be messed up by errors in the initial representation. Erroneous space perception is thus expected for the far-tonear integration.

\section{The continuity assumption}

The SSIP hypothesis argues that surface geometry is computed by tracing continuous changes along the surface, rather than inferring absolute parameters directly. Thus continuous surface information is necessary for executing the integrative process and the computation will break down when a discontinuity in surface information is found. Consider the example in Figure 4.4(a). The occlusion of the wall causes a jump in 
depth between $S_{1}$ and the visible area of $S_{2}$. The visual system needs to recover the invisible area, instead of advancing the integrative process on $S_{1}$ to the visible area of $S_{2}$. The process of representing $S_{1}$ thus breaks down at the place the wall erects. And $S_{2}$ is represented by another integrative process that bases solely on depth information on it. The second process will be hampered by an inaccurate initial representation. For example, its location may also be erroneously registered because $P$ and $P^{\prime}$, which locate on the same optical contact line, tend to appear equidistant (Gogel, 1965, 1990). Also, owing to the "frontal shift" tendency in surface slant perception, $\mathrm{S}_{2}$ will be misperceived as being slanted upward. As a result, the distance to an object behind wall is underestimated.

Similarly, a sudden change in the ground texture (Figure 4.4b) also prevents the integrative process on $S_{1}$ from continuing on $S_{2}$. $S_{2}$ is judged using the information on it and distance is underestimated when the "frontal shift" tendency biases the perception of $\mathrm{S}_{2}$. For a misperceived slant of $\delta$, distance is judged as $d^{\prime}=d_{1}+d_{2} \sin (\alpha) / \sin (\alpha+\delta)$ (Equation 4.3), where $d_{1}$ and $d_{2}$ are actual distances along the $S_{1}$ and $S_{2}$ and $\alpha$ specifies the angular declination of the target with respect to the eye level.

\subsection{The plan to assess the SSIP hypothesis}

Of three propositions, we will first examine how continuity of ground information affects space perception. Provided that texture is a significant source of depth information within action space and our previous experiments in the real world (Sinai et al., 1998; Yarbrough et al., 2002; He et a1., 2004) have shown that a continuous texture on the ground is critical for accurate distance judgment, next chapter we will further examine 
this issue in virtual reality (VR). Both the occlusion effect and the texture discontinuity effect on distance perception will be investigated. Mathematically, Equation 4.3 will be quantitatively examined.

The significance of the near ground area will be examined in the real-world experiments reported in Chapter 6. There the subject's view of the ground was limited by wearing a pair of Field-Of-View (FOV) restricting goggles. By changing the size of FOV, the accessibility of the near ground area was manipulated, and the role of near ground area on absolute and relative distance judgment (Equation $4.1 \& 4.2$ ) was assessed.

The directional asymmetry hypothesis will also be tested in Chapter 6 by letting subjects scan the target distance either from near to far or in the reverse direction. According to the SSIP hypothesis, we expect an accurate ground representation and veridical distance judgment in the near-to-far scan, but not in the reverse direction. 


\section{CHAPTER 5}

\section{GROUND-BASED DISTANCE PERCEPTION IN VIRTUAL REALITY}

The problem addressed here is how continuity of texture information on the ground influences the representation of the ground surface and hence the perception of distance. As discussed in the last chapter, the SSIP hypothesis asserts that continuous texture information is crucial for the integrative process of representing the ground. Thus a sudden change in the ground texture will stop the integrative process and introduce errors in the ground representation (see Figure 4.4 for details). To assess this idea, subjects were tested in immersive VR environments, in which the ground texture could be easily and precisely manipulated. Exp 5.1 compared subjects' distance judgments in the conditions that the ground was homogenously textured or separated into two regions by different textures. Exp 5.2 then aimed to reveal the mechanism underlying the texturediscontinuity effect. The ground representation error caused by texture discontinuity was assessed and related to distance judgments using Equation 4.3. Exp 5.3 further illustrated the significance of a continuous ground in space perception: distance was underperceived when the ground was partially occluded by some obstacle. The last experiment was to evaluate an alternative explanation for our findings - the 'equidistance tendency' hypothesis (Gogel, 1965) 


\subsection{The virtual reality system and virtual environments}

To create controllable experimental environments, the research was carried out in an HMD-based VR system with head-tracking and stereo display. The system consists of a Dell Precision 350 Workstation with an nVidia Quadro4 XGL900 graphics card, coupled with an Intersense's IS-600 Mark2 tracking system (6-DOF tracking with a sampling rate up to $150 \mathrm{~Hz})$ and a Virtual Research Systems' V8 $\mathrm{HMD}\left(60^{\circ}\right.$ diagonal field of view, $640 \times 480$ pixel resolution). The EAI's WorldUp software is used to run virtual environments. It creates an immersive virtual environment by continuously tracking the viewer's location and heading orientation and accordingly updating the HMD's display of the virtual world from a correct viewpoint in real time. A striking impression of depth is produced by motion parallax and pictorial cues with or without binocular disparity in stereoscopic or non-stereoscopic views.

A typical virtual environment in this research had a blue sky, a flat, textured ground, a stimulus target in front of the observer and a matching target behind him. On a given experimental trial, the stimulus target was set at a particular distance and the observer was instructed to move the matching target, a red cross, until distances to both targets appeared equal. A short yellow line was drawn on the ground to tell the observer his position in the virtual world.

\subsection{Exp 5.1. Distance underestimation across discontinuous textures}

The texture-discontinuity effect on distance perception was investigated. In the virtual world, the ground was textured homogenously with grass, or it consisted of two regions that were distinguished by different textures (grass/cobblestones), as shown in the 
Figure 5.1. As we had discussed before, the SSIP hypothesis predicted distance underestimation in the latter condition.

\subsubsection{Methods}

Observers. Eight paid naïve subjects were tested. All of them have normal or correct-to-normal visual acuity.

Design. Three independent variables were used in a cross-factor design with 2 measures for each combination of factors. These factors were: (1.) the continuity of the ground texture (homogenous texture (grass) or different textures (grass/cobblestones)), (2.) the target's distance from the viewer $(5 \mathrm{~m}, 7 \mathrm{~m}, 9 \mathrm{~m}$, and $11 \mathrm{~m})$, and (3.) the display modes (stereoscopic or non-stereoscopic views). Each subject was tested with 32 trials (2 texture-continuity conditions $\times 4$ distances $\times 2$ display modes $\times 2$ replications). The presentation of experimental trials was blocked by different display modes, but fully randomized within each block.

Procedure. Subjects were tested individually. Their eye heights were measured before the test and used for calibrating the rendering geometry of the VR system. After reading the instruction, subjects wore the HMD and were shown the virtual world. They were asked to look around and walk around in the virtual environment so that they could quickly accustom themselves to it.

Subjects completed 12 practice trails first. The practice trials utilized the same procedure as that in the test. Each trial started with the calibration. The subject was asked to face the $+Z$ direction so that the coordinate system of the virtual world was aligned with that of the real world. The virtual world was then shown. The perceptual matching paradigm was used to measure the perceived distance. On a given trial, the subject first 
faced the stimulus target. He was asked to estimate and remember how far the target was. And then he turned around and faced the matching target that was initially set at a random location. Using the arrow keys on the keyboard, he could move the matching target nearer or farther. The instruction was to relocate the matching target until it appeared to be the same distance from the subject as the stimulus target. To make an accurate judgment, subjects were encouraged to turn back and forth and repeatedly examine both targets. Usually, the duration of one trial was less than 2 minutes, and the entire block required about 25 minutes.

\subsubsection{Results}

As shown in Figure 5.2, the experiment replicated the Sinai et al's (1998) finding. The same response pattern was found in the stereoscopic and non-stereoscopic display modes: distance was judged shorter when the ground was divided into two differentlytextured regions than it was homogeneously textured. For example, the distance of $11 \mathrm{~m}$ was accurately matched on a continuous ground, but underestimated in the texturediscontinuity trials by $0.71 \mathrm{~m}$ for the stereoscopic display $(11.15 \pm 0.30 \mathrm{~m}$ in the control condition vs. $10.44 \pm 0.34 \mathrm{~m}$ in the texture-discontinuity condition) and by $0.90 \mathrm{~m}$ for the non-stereoscopic display $(11.06 \pm 0.33 \mathrm{~m}$ in the control condition vs. $10.16 \pm 0.38 \mathrm{~m}$ in the texture-discontinuity condition). In addition, although distance underestimation caused by the discontinuity in ground texture pattern was slight larger in the non-stereoscopic display condition than in the stereoscopic display condition, a three-way repeatedmeasures ANOVA (Display $\mathrm{x}$ Distance $\mathrm{x}$ Surface-continuity) found that slight difference was insignificant (main effect: $F(1,7)=1.904, p=0.207$; the interaction of (Display $x$ Distance): $F(3,21)=0.876, p=0.469$; the interaction of (Display $x$ Surface-continuity): 
$\mathrm{F}(1,7)=1.439, \mathrm{p}=0.269$; interaction of (Display $\mathrm{x}$ Distance $\mathrm{x}$ Surface-continuity): $F(3,21)=1.238, p=0.321)$.

As expected, the ANOVA found significant main effect of texture continuity $(F(1,7)=36.113, p<0.001)$. The interaction of (Distance $x$ Texture-continuity) was also significant $(F(3,21)=4.170, P<0.02)$. Because the texture boundary was experimentally placed halfway between the observer and the target, the significant interaction suggested that with increasing distance, a larger area behind the boundary caused more distance. In sum, the results are in agreement with the prediction from the SPSS hypothesis.

\subsection{Exp 5.2. The ground representation errors due to texture discontinuity}

The purpose of this experiment is twofold. First, to reveal that it is discontinuity in texture information, not difference in texture patterns, that causes distance underestimation, subjects' distance perception was measured when the ground was tiled with same checkerboards or divided by two checkerboard patterns (Figure 5.3). In the latter condition, two kinds of checkerboards were identical in spatial structure but differently colored. Thus distance underestimation, if found, could not be attributed to difference between checkerboard patterns. Furthermore, the presentation order of two kinds of checkerboards was switched to negate this possibility.

Second, the SSIP hypothesis explains the texture-discontinuity effect by the "frontal shift" bias in representing the ground surface. For example, if the ground is segmented into distinct areas by different textures, the surface representation process initiated from near space will break up at the texture boundary. The surface beyond the boundary is thus perceived solely from the information on it and the "frontal shift" effect 
will bias the perceived surface upward. Accordingly, distance is underperceived: $d^{\prime}=d_{1}+d_{2} \sin (\alpha) / \sin (\alpha+\delta$ ) (Eq. 4.3). To assess this, the ground representation error was measured in the texture-discontinuity condition. According to the SSIP hypothesis, we expected that the ground representation error and distance underestimation could be quantitatively related by using the above equation.

\subsubsection{Methods}

Observers. The same eight subjects participated in this experiment.

Stimuli. The VR world was presented stereoscopically so that both binocular disparity and monocular cues could be used to judge surface orientation. The ground was divided into two regions by differently-colored checkerboard patterns, as shown in Figure 5.3. Two checkerboard patterns were identical in spatial structure but displaced with $90^{\circ}$ phase shift. In addition, to prevent the observer from using the horizon to judge surface orientation, a wall was set to occlude it. Also the subject was clearly instructed that the wall would change position randomly from trail to trail so it could not be used as the reference in distance or slant judgments.

Design. For the distance judgment task, the cross-factor design had three independent variables: (1.) the presentation order of checkerboard patterns (magenta/cyan checkerboard at near and tan/light-wheat checkerboard at far, or in reverse), (2.) the location of the texture boundary (uniform texture, or texture boundary at $3 \mathrm{~m}$ or $5 \mathrm{~m}$ ) and (3.) the target distance $(7 \mathrm{~m}, 9 \mathrm{~m}$, and $11 \mathrm{~m})$. Each combination of these factors was measured with 2 replications and the total trail number was 36 (2 texture orders $\times 3$ boundary locations $\times 3$ target distances $\times 2$ replications). In the experiment, the presentation of trials was fully randomized. 
For the slant perception task, two independent variables were used in a crossfactor design with 4 measures for each combination of factors. These factors were: (1.) the presentation order of texture patterns (same as the distance judgment task) and (2.) the location of the texture boundary $(3 \mathrm{~m}, 4 \mathrm{~m}$, or $5 \mathrm{~m}$ from the observer). Each subject was tested with 24 trials ( 2 texture order $x 3$ boundary locations $x 4$ replications).

Procedure. Distance judgment was measured using the same perceptual matching paradigm as in the previous experiment.

On a given trial of the slant perception task, the initial orientation of the distant ground area was randomly set to a value between $12^{\circ}$ downward and $12^{\circ}$ upward and the near ground was always level. The subject was asked to compare two ground areas and rotate the distant area upward or downward until it seemed to be coplanar with the near one. To prevent them from judging the relative slant using the side view, subjects were not allowed to turn left or right. Usually, each trial took less than 1 minute and the entire block required about 25 minutes.

\subsubsection{Results}

First, since two kinds of checkerboards were identical except for their colors, the experiment found no effect of the texture presentation order on both slant and distance judgments. For slant judgments, a two-way repeated-measures ANOVA (Texture order $\mathrm{x}$ Boundary location) found both main effect of texture order $(F(1,7)=0.002, p=0.968)$ and the interaction of (Texture order $\mathrm{x}$ Boundary location) $(\mathrm{F}(2,14)=0.603, \mathrm{p}=0.561)$ were insignificant. Distance judgment was also little affected by different texture orders (main effect: $F(1,7)=4.125, p=0.082$; the interaction of (Distance $x$ Texture order): $\mathrm{F}(2,14)=1.304, \mathrm{p}=0.302$; the interaction of (Texture order $\mathrm{x}$ Boundary location): 
$\mathrm{F}(2,14)=0.603, \mathrm{p}=0.561$; the interaction of (Distance $\mathrm{x}$ Texture order $\mathrm{x}$ Boundary location): $F(2,14)=1.777, p=0.205)$. Thus, in the following analysis the data from different texture orders were pooled together.

The experiment confirmed the texture discontinuity effect (Figure 5.4). Significant distance underestimation was found when the ground was textured with different kinds of checkerboards $(F(1,7)=24.419, \mathrm{p}<0.005$ in the texture-boundary-at-3m condition; $F(1,7)=35.655, \mathrm{p}<0.001$ in the texture-boundary-at- $5 \mathrm{~m}$ condition). It should be noted that the effect was not caused by any difference in texture patterns, because both kinds of checkerboards had identical spatial structure and thus produced the identical texture gradients. Also, when we switched the presentation order of checkerboard patterns, distance underestimation was almost the same. Therefore, we may conclude that, it is the change in texture pattern, i.e. discontinuity in texture pattern, that introduces errors in distance perception.

Also, errors were found in the slant judgment task. All subjects showed the same pattern in their responses: the distant ground area was misperceived as being slanted upward. When the distant region was reported to be perceptually level, it in fact slanted downward by about 1 degree $\left(1.28^{\circ} \pm 0.37^{\circ}, 1.16^{\circ} \pm 0.36^{\circ}\right.$, and $1.08^{\circ} \pm 0.24^{\circ}$ for the texture boundary located at $3 \mathrm{~m}, 4 \mathrm{~m}$, and $5 \mathrm{~m}$, Figure 5.5$)$.

As shown in Figure $5.4 \&$ 5.5, subjects' errors in slant and distance judgments could be related by using Equation 4.3. Consider the ground representation error in the texture discontinuity condition. Errors estimated from distance judgment quantitatively matched well with those from the slant judgment task and no significant difference was found (for the texture boundary located at $3 \mathrm{~m}: \mathrm{t}(7)=1.699, \mathrm{p}=0.133$; for the texture 
boundary located at $5 \mathrm{~m}: \mathrm{t}(7)=1.591, \mathrm{p}=0.156)$. These results undoubtedly establish that distance underestimation can be attributed to the misrepresented ground surface as the SPSS hypothesis suggests.

\subsection{Exp 5.3. The occlusion effect on distance judgment}

The current experiment examined the occlusion effect on distance judgment. The experimental VR environments are shown in Figure 5.6. The ground was an open field of grass, or it was partially occluded by a short wall $(0.5 \mathrm{~m}$ (Height) $\mathrm{x} 1 \mathrm{~m}$ (Length) $\mathrm{x} 10 \mathrm{~m}$ (Width)) that lay halfway between the observer and the target. According to the SSIP hypothesis, distance would be underestimated when a continuous ground was partially occluded.

\subsubsection{Methods}

Observers. The same eight subjects were tested.

Design. The cross-factor design was the same as in Exp 5.1. Three independent factors were: (1.) the continuity of the ground surface (continuous vs. partially-occluded), (2.) the target's distance from the observer $(5 \mathrm{~m}, 7 \mathrm{~m}, 9 \mathrm{~m}$, and $11 \mathrm{~m})$, and (3.) the display modes (stereoscopic or non-stereoscopic views). Each subject was tested with 32 trials (2 surface-continuity conditions $\times 4$ distances $\times 2$ display modes $\times 2$ replications). The presentation of experimental trials was blocked by different display modes, but fully randomized within each block.

Procedure. The experimental procedure was the same as in Exp 5.1. 


\subsubsection{Results}

Distance judgments in the control and occlusion conditions, averaged across 8 subjects, are shown in Figure 5.7. Clearly, target distances were underestimated in the occlusion condition. A three-way repeated-measures ANOVA (Display $\mathrm{x}$ Distance $\mathrm{x}$ Surface-continuity) found a significant main effect of surface-continuity $(F(1,7)=21.113$, $\mathrm{p}<0.002)$ and a significant interaction of (Distance $\mathrm{x}$ Surface-continuity) $(\mathrm{F}(3,21)=$ $5.384, \mathrm{p}<0.01)$

Similar to Exp 5.1, slight difference due to different display modes was not statistically significant (main effect: $F(1,7)=1.904, p=0.207$; the interaction of (Display $x$ Distance): $F(3,21)=0.876, p=0.469$; the interaction of (Display $x$ Surface-continuity): $F(1,7)=1.439, p=0.269$; the interaction of (Display $x$ Distance $x$ Surface-continuity): $\mathrm{F}(3,21)=1.238, \mathrm{p}=0.321)$. This pointed out that binocular depth information was not the source of distance underestimation. Instead, since the wall was placed halfway between the observer and the target and hence larger ground area became invisible when the wall moved away from the observer along with the target, the aforementioned significant interaction of (Distance $x$ Surface-continuity) thus illustrated the trend that distance underestimation resulted from the destruction of a continuous ground surface and increased with larger occlusion.

Overall, the prediction from the SSIP model was confirmed: surface layout cannot be accurately registered if the texture continuity presumption is violated. 


\subsection{Exp 5.4. SSIP or "equidistance tendency"?}

An alternative hypothesis for the previous results is the 'equidistance tendency' in the perception of distance (Gogel, 1963, 1990). Gogel and others have shown that in reduced viewing conditions and sometimes well-structured natural environments, the relative depth between two objects tends to be underperceived and thus people judge them to be the same distance away. So one may explain the occlusion effect with the possibility that the target is perceptually drawn toward the wall. Also, the texturediscontinuity effect can be accounted for by the perceptually compressed space between the boundary and the target.

The experiment was to examine this hypothesis. The experimental setting in VR was similar to that in the Experiment 1, except that the wall was placed behind the target (Figure 5.8(a)). As predicted by the equidistance-tendency hypothesis, if the equidistance tendency operates, two objects in the scene will be perceptually grouped together and distance to the target overestimated. On the other hand, according to the SSIP model, no systematical error in distance judgment should be expected because a continuous ground could be seen from the viewer to the target. Here, to favor the 'equidistance tendency' hypothesis, we tested subjects with the cue-reduced non-stereoscopic views.

\subsubsection{Methods}

Observers. The same eight subjects were tested.

Design. The cross-factor design and the presentation of experimental trials were the same as in Exp 5.3. Two independent variables were: (1.) the appearance of the wall in the scene (with or without wall) and (2.) the target's distance $(5 \mathrm{~m}, 7 \mathrm{~m}, 9 \mathrm{~m}$, and $11 \mathrm{~m}$ ).

Procedure. The procedure was the same as in Exp 5.3. 


\subsubsection{Results}

Clearly, no distance overestimation was shown in Figure 5.8(b). Instead of being pulled away by the wall at the back, distance estimates were similar in the control condition and wall-at-the-back conditions, although the cue-reduced display might favor the 'equidistance tendency'. A two-way repeated-measures ANOVA (Distance $\mathrm{x}$ Wallexistence) found an insignificant main effect of wall existence $(F(1,7)=0.037, p=0.853)$ and an insignificant interaction of (Distance $x$ Wall-existence) $(F(3,21)=2.981, p=0.055)$. Thus, the occlusion effect cannot be explained as the 'equidistance tendency' in distance perception. Instead, consistent with the prediction of the SPSS hypothesis, the results show that the distance is accurately judged along a continuous ground.

\subsection{Summary}

The above results prove the critical role of continuous ground information in space perception. Consistent with the SSIP hypothesis, the perception of distance was found underestimated when the ground was partially occluded by a wall or it was segmented into two regions by different textures. More important, errors in the ground representation were quantitatively related to distance underestimation: observers did misperceive the surface beyond the texture boundary and misjudge it as being slanted upward. The results reinforce the idea underlying the SSIP hypothesis that veridical space perception relies on an integrative process that runs along a continuous surface. 


\section{CHAPTER 6}

\section{GROUND REPRESENTATION FROM AN INTEGRATIVE PROCESS}

This study aims to investigate the mechanism that integrates local ground information to form a global surface representation. According to the aforementioned SSIP hypothesis, an accurate representation of the ground can be built by sampling and combining depth information over space and time, and the computation may start from near space where rich depth cues can provide for a reliable initial representation and then progressively extend to distant areas. To assess this model, three propositions from the SSIP hypothesis will be examined: (1). Information from an extended ground area is required for yielding an accurate ground representation and consequently accurate distance judgment. (2). The near ground area about the observer has a critical role in the computation because it affords a reliable initial surface representation. (3). The directional asymmetry is expected in the integrative process. A near-to-far integration can produce a veridical global representation of the ground by gradually adding distant surface areas into an initial representation that has been accurately perceived. In contrast, an erroneous result is likely for the far-to-near integration, given that it is implausible to yield a correct initial representation of a distant ground area from pictorial depth cues only. 
Eight experiments were carried out in the real environments. Experiments $6.1 \&$ 6.2 investigated the first proposition, which systematically examined how the perception of absolute and relative distance was affected by restricting an observer's field of view (FOV). The following three experiments investigated the second proposition. The problem addressed was which part of the ground holds essential information for building an accurate ground surface representation. Experiments $6.6 \& 6.7$ examined the directional asymmetry hypothesis. With a restricted FOV, subjects were asked to judge the distance to a target by scanning it from near to far, or in the reversed direction. The SSIP hypothesis suggested that accurate distance judgment would be found only in the near-to-far scan condition. The last experiment was a control experiment conducted in the dark. The objective was to negate the alternative explanation that the directional asymmetry in distance judgment might result from the head-motion signal from scanning rather than the surface integration process.

\subsection{Exp 6.1. Absolute distance judgment with restricted FOV}

The current experiment was to examine the hypothesis that space perception bases on a global representation of the ground surface. Subjects' accessibility to the global structure of the ground was carefully controlled by restricting their $\mathrm{FOV}^{[*]}$ to a small size. Clearly, because only local depth information from the ground area about the target is available, the perception of distance will be impaired in the FOV-restricted conditions if the large-scale ground structure is necessary for veridical space perception.

[*] The normal FOV of human visual system expands about 200 degrees horizontally, taking into account both eyes, and 135 degrees vertically (Werner, 1991). 


\subsubsection{Methods}

Observers. Eight paid naïve subjects with normal vision participated in the experiment. All have a right dominant eye.

Experimental Setting and Stimuli. The experiment was conducted outdoors on a flat, grassy lawn about $60 \mathrm{x} 90 \mathrm{~m}$. The target was a red hockey puck (3" in diameter \& 1" in height) placed at the preset distance. During the experiment, subjects wore a pair of FOV-restricting goggles (Figure 6.1) - a pair of black-painted safety goggles with a transparent window before the right eye. Their FOV could be set to particular sizes and shapes by using different viewing windows.

Design. Two independent variables were used in a cross-factor design with 2 measures for each combination of factors: (1.) different viewing windows and (2.) the target's distance from the observer $(4,5,6$, and $7 \mathrm{~m})$. Three rectangular viewing windows were used, which limited the observer's FOV, averaged across 8 subjects, to $\left(13.9^{\circ} \times 13.5^{\circ}\right.$, $21.2^{\circ} \times 21.2^{\circ}$, and $\left.38.6^{\circ} \times 39.5^{\circ}\right)$. A total of 24 trials were tested with the reduced FOV ( 3 viewing windows $\times 4$ distances $\times 2$ replications). The experiment was blocked by different FOVs, but was randomized in the presentation of trials within each block. In addition, a baseline measure was performed without the FOV-restricting goggles and with the non-dominant eye patched.

Procedure. Subjects were tested individually. After reading the instruction, subjects completed four practice trials to familiarize them with the procedure. Then the baseline measure was performed with the normal FOV first and followed by the FOVrestricted blocks. The testing order of the FOV-restricted blocks was balanced through subjects. 
At beginning of each trial, the subject was blindfolded and led to the starting position. The experimenter then carefully positioned the subject's head so that when he removed the blindfold, the target was visible in his FOV. The subject was asked to judge the target distance without head movement. The perceived distance was measured using the blindfolded walking paradigm: The subject viewed the target, put on the blindfold again, and then walked quickly and decisively to where he thought the target was. After the subject stopped, the experimenter measured the walked distance and led the subject back to the starting point with eyes still blindfolded. The duration of each trail averaged about $2 \mathrm{~min}$. No error feedback was provided in the experiment.

\subsubsection{Results}

The perception of distance with different FOVs, averaged across 8 subjects, is shown in Figure 6.2(b). Consistent with previous studies (Hagen et al., 1978; Dolezal, 1982), the results found that restricting FOV to $20^{\circ}$ or less could degrade subjects' distance perception. Compared to the full-view condition, subjects significantly underperceived the distance to the target with a FOV of $\left(13.9^{\circ} \times 13.5^{\circ}\right)$ and $\left(21.2^{\circ} \times 21.2^{\circ}\right)$ (the main effect of reduced FOV: $F(1,7)=60.875, p<0.001$ for the FOV of $\left(13.9^{\circ} \times 13.5^{\circ}\right)$; $F(1,7)=18.276, p<0.005$ for the FOV of $\left.\left(21.2^{\circ} \times 21.2^{\circ}\right)\right)$

However, when the FOV was large enough $\left(38.6^{\circ} \times 39.5^{\circ}\right.$ in the experiment), subjects could judge distance accurately and their estimates were similar to the full-view baseline condition (the main effect of reduced FOV: $F(1,7)=0.012, p=0.916$; the interaction of (FOVs $x$ Distance): $F(3,21)=0.370, p=0.775)$. That is, veridical distance judgment was possible only if a wide FOV made extended ground area visible and large- 
scale information of the ground available. This suggests that, to represent the ground and distances accurately, information over an extended ground area is required.

\subsection{Exp 6.2. Relative distance judgment with restricted FOV}

This experiment measured the influence of restricting FOV on subjects' judgment of relative distance. Given that visual space is built on the ground representation, we predicted that subjects' judgment of absolute and relative distance would be influenced in the same way if the ground surface were misrepresented in the FOV-restricted conditions. More mathematically, provided that the ground representation error due to restricting FOV could be inferred from absolute or relative distance judgment using Equation $4.1 \&$ Equation 4.2 (see Chap 4.3 for details), we expected a correlation between the estimated representation errors from two tasks.

\subsubsection{Methods}

Observers. The same eight subjects participated in the experiment.

Design. The same cross-factor design was used as in the previous experiment. Two factors were (1). subjects' FOVs $\left(13.9^{\circ} \times 13.5^{\circ}, 21.2^{\circ} \times 21.2^{\circ}\right.$, or $\left.38.6^{\circ} \times 39.5^{\circ}\right)$ and (2). the location of the stimuli $(5,6$, or $7 \mathrm{~m}$ from the observer). Each combination of factors was measured with at least four replications. In the experiment the presentation of trials was blocked by different FOVs, but fully randomized within each block.

Also, a baseline measure was performed monocularly with full field of view.

Procedure. Subject's judgment of relative distance was measured using the Lshape matching paradigm. The L-shaped stimulus was constructed of two white pipes, $30 \mathrm{~mm}$ in diameter. Its frontal arm was $40.5 \mathrm{~cm}$ long and its depth arm was adjustable 
$(30 \sim 176 \mathrm{~cm})$. The subject's task was to compare two arms and then instruct the experimenter to shorten or lengthen the depth arm until both arms were judged as being of the same physical length. To prevent the subject from being able to use the experimenter's body as the reference, the experimenter would stay outside the subject's FOV all the time and the subject was blindfolded while the L-shape was being adjusted.

\subsubsection{Results}

Subjects' judgment of relative distance, measured by the aspect ratio of the Lshape ( $r=($ the width of the frontal arm)/(the length of the depth arm)) when it was perceived square, was shown in Figure 6.3(b). First of all, like previous studies (Harway, 1963; Toye, 1986; Loomis et al., 1992; Loomis et al., 1996; Loomis \& Philbeck, 1999), perceptual foreshortening in relative distance perception was found. Take the full-view condition as the example. The depth arm was $50.1 \% \sim 63.4 \%$ longer than the frontal arm when a square L-shape was judged, although the instruction gave emphasis to the matching of physical lengths. And thus the matched $r$, ranging from 0.62 to 0.68 , deviated from the theoretical value of $r=1$ for an isotropic matching.

Second, relative distance judgment was significantly influenced by the L-shape's location and the size of FOV. The overall tendency was that perceptual foreshortening increased as the L-shape was moved away from the observer or the observer's FOV was reduced. A two-way repeated measures ANOVA (FOVs x Location) yielded significant main effects of the reduced FOV $(\mathrm{F}(3,21)=41.607, \mathrm{p}<0.001)$ and L-shape's location $(F(2,14)=34.104, p<0.001)$, as well as a significant interaction of (FOVs $x$ Location) $(\mathrm{F}(6,42)=3.825, \quad \mathrm{p}<0.005)$. 
Same as the previous experiment, different FOVs generated different response patterns. With a FOV of $\left(38.6^{\circ} \times 39.5^{\circ}\right)$, subjects' judgment was similar to that in the fullview condition (the main effect of reduced FOV: $F(1,7)=0.013, p=0.914$; the interaction of (FOVs x Location): $\mathrm{F}(2,14)=0.077, \mathrm{p}=0.926)$. But foreshortening became significantly larger when the FOV was reduced to $\left(13.9^{\circ} \times 13.5^{\circ}\right)$ and $\left(21.2^{\circ} \times 21.2^{\circ}\right)$ (the main effect of reduced FOV: $F(1,7)=60.017, p<0.001$ for the FOV of $\left(13.9^{\circ} \times 13.5^{\circ}\right) ; F(1,7)=17.001$, $\mathrm{p}<0.005$ for the FOV of $\left(21.2^{\circ} \times 21.2^{\circ}\right)$; the interaction of (FOVs $x$ Location): $F(2,14)=26.809, p<0.001$ for the FOV of $\left(13.9^{\circ} \times 13.5^{\circ}\right) ; F(2,14)=35.865, p<0.001$ for the FOV of $\left.\left(21.2^{\circ} \times 21.2^{\circ}\right)\right)$

Clearly, the influence of restricting FOV on relative distance judgment is in some sense consistent with that on absolute distance judgment. Both tasks were significantly affected only if the observer's FOV was reduced to $20^{\circ}$ or less. The similarity indicates a common mechanism for both types of space judgment: errors came from the misrepresentation of the ground due to the restricted FOV. To further confirm this possibility, errors in the ground representation were derived from both tasks using equations $4.1 \& 4.2\left(d^{\prime}=d^{*} \sin (\alpha) / \sin (\alpha+\delta)\right.$ and $r=\sin (\alpha-\Delta \alpha) / \sin (\alpha-\Delta \alpha+\delta)$ for absolute and relative distance judgment, respectively, see Figure 4.3(a) for details). As shown in Figure 6.4, the errors estimated from both judgments increased similarly with reducing FOV and a strong correlation was found $\left(\mathrm{R}^{2}=0.9018, \mathrm{p}<0.001\right)$. That is, when less (more) ground information becomes available with a smaller (larger) FOV, the accuracy of representing the ground surface decreases (increases) and consequently distance judgments, absolute and relative, get worse (better). 


\subsection{Exp 6.3. Judging distances with vertically restricted FOV}

The above experiments have established that space perception improves with larger FOV as the visual system is able to access more information for representing the ground surface. The questions to be further examined are: what information is essential for representing the ground accurately and which part of the ground holds such information?

For these questions, the SSIP hypothesis asserts a significant role of the near ground area because it can afford a reliable initial surface representation. This and the following two experiments are to examine this idea. In this experiment, we carefully controlled the appearance of the near ground area in the subject's FOV. If information on the near ground surface was vital for building an accurate ground surface representation, absolute distance judgments would be degraded without access to the near ground area.

\subsubsection{Methods}

Observers. Five old subjects and three new ones were tested. All of them have normal or correct-to-normal vision. And all have a right dominant eye.

Design. The same cross-factor design was used as in the previous experiments. Two factors were (1.) different viewing windows and (2.) the target's distance from the observer $(4,5,6$, and $7 \mathrm{~m})$. Four rectangular viewing windows were used, whose width and height were $32 \mathrm{~mm}$ and $(2.5,8,14$, or $22 \mathrm{~mm})$. They limited the observer's FOV, averaged across 8 subjects, to $\left(>57.7^{\circ}\right.$ horizontally $\mathrm{x}\left(13.6^{\circ}, 21.1^{\circ}, 29.6^{\circ}, 39.9^{\circ}\right)$ vertically). A total of 32 trials were tested with the reduced FOV (4 viewing windows $x 4$ distances $\times 2$ replications), and the presentation of these trials was blocked by different 
FOVs but randomized within each block. In addition, a baseline measure was performed monocularly with full field of view.

Procedure. Subjects' estimates of the distance to the target were measured by using the blindfolded walking paradigm described in the Exp 6.1.

\subsubsection{Results}

As shown in Figure 6.5(b), narrowing FOV vertically influenced subjects' estimates of distance. Subjects tended to walk shorter distances in the FOV-restricted conditions as compared to the full-view condition. A two-way repeated measures ANOVA (FOVs $x$ Distance) yielded a significant main effect of the reduced vertical FOV $(\mathrm{F}(4,28)=28.394, \mathrm{p}<0.001)$ as well as a significant interaction of (FOVs $\mathrm{x}$ Distance) $(\mathrm{F}(12,84)=2.415, \mathrm{p}<0.010)$

Moreover, overall underestimation of distance was significant only when the vertical viewing field was reduced to $20^{\circ}$ or less, i.e. with the vertical FOVs of $13.6^{\circ}$ and $21.1^{\circ}$ only (the main effect of reduced FOV: $F(1,7)=69.420, p<0.001$ for the vertical FOV of $13.6^{\circ} ; \mathrm{F}(1,7)=27.220, \mathrm{p}=0.001$ for the vertical FOV of $21.1^{\circ}$ ). No significant underestimation of distance was found with the vertical FOVs of $29.6^{\circ}$ and $39.9^{\circ}$ (the main effect of reduced FOV: $F(1,7)=4.151, p=0.081$ for the vertical FOV of $29.6^{\circ}$; $F(1,7)=1.473, p=0.264$ for the vertical FOV of $\left.39.9^{\circ}\right)$.

Note that a viewing window makes only the ground area in the vicinity of the target visible and expanding FOV vertically can make the near ground area visible. For example, for a target distance of $7 \mathrm{~m}$ and an average eye height of $1.67 \mathrm{~m}$, a vertical window of $13.6^{\circ}$ blocked the view of the near ground surface up to $3.28 \mathrm{~m}$. Reliable depth information within near space thus was missed in distance judgment. On the other hand, 
through a viewing window of $39.9^{\circ}$, distance was perceived accurately for the reason that the ground became visible at $1.24 \mathrm{~m}$. The analysis thus indicates that reliable depth information from the near ground surface is valuable for accurately perceiving the ground surface and distance along it.

\subsection{Exp 6.4. Judging distances with horizontally narrowed FOV}

The experiment was to further illustrate that the near ground area holds essential information for accurate space perception. Subjects were exposed to horizontally narrowed FOVs that had a vertical visual field of $50.9^{\circ}$ and thus made the near ground area visible. According to the SSIP hypothesis, we expected accurate distance judgment in these conditions because the ground could be represented accurately with access to information about the near ground area.

\subsubsection{Methods}

Observers. The same eight subjects were tested.

Design. The same cross-factor design was used as in the previous experiment. Four viewing windows were used, which limited the observer's FOV, averaged across 8 subjects, to $\left(\left(14.3^{\circ}, 21.5^{\circ}, 29.2^{\circ}, 39.9^{\circ}\right)\right.$ horizontally $\mathrm{x} 50.9^{\circ}$ vertically $)$.

Procedure. The same blindfolded walking paradigm was used as in the previous experiment.

\subsubsection{Results}

For vertical viewing windows, narrowing the horizontal FOV showed little effects on the perception of distance. Subjects' estimates of distance with or without a vertical viewing window were nearly identical, as shown in Figure 6.6(b). A two-way repeated 
measures ANOVA found an insignificant main effect of the narrowed horizontal FOV $(\mathrm{F}(4,28)=0.143, \mathrm{p}=0.965)$ as well as an insignificant interaction of (FOVs $\mathrm{x}$ Distance) $(\mathrm{F}(12,84)=0.863, \mathrm{p}=0.110)$.

Consider how two types of FOV influenced subjects' distance judgment and how they manipulated the access to the near ground information. As mentioned before, the vertically-restricted FOV of $13.6^{\circ}$ severely diminished subjects' access to the near ground information by blocking the view of the ground up to $3.28 \mathrm{~m}$. In this condition, distance was underestimated. On the other hand, if the viewing window was rotated by 90 degree, a FOV of $\left(14.3^{\circ} \times 50.9^{\circ}\right)$ made the near ground visible from $0.8 \mathrm{~m}$ and consequently gave accurate distance judgment. The results thus confirm the notion that the near ground area holds essential information for accurate space perception.

\subsection{Exp 6.5. Accurate distance judgment with free head movement}

So far, we have shown the impact of the near ground information on distance judgment. Here we further examined the hypothesis that distance could be accurately judged by sampling and integrating the ground information across space. In this experiment, the subjects were tested with restricted FOVs. They were instructed to examine the path between their feet and the target several times when judging the distance. According to the SSIP hypothesis, accurate distance perception was anticipated because of access to the near ground information and the accumulation of ground information across space.

\subsubsection{Methods}

Observers. The same eight subjects were tested. 
Design. The cross-factor design was the same as in Exp 6.4. Two independent variables were: (1.) different FOVs $\left(57.7^{\circ}\right.$ horizontally x $\left(13.6^{\circ}, 21.1^{\circ}, 29.6^{\circ}, 39.9^{\circ}\right)$ vertically) and (2.) the target's distance from the observer $(4,5,6$, and $7 \mathrm{~m})$. Subjects were tested with 32 trials (4 window sizes $\times 4$ distances $\times 2$ replications). The presentation of experimental trials was blocked by different viewing windows, but fully randomized within each block.

Procedure. The procedure was the same as in Exp. 6.3 except that, instead of judging the distance from a static view, the subject was asked to move his head, look back and forth, and scan the path between his feet and the target several times.

\subsubsection{Results}

By repeatedly scanning the target distance, subjects made accurate estimates and the restricted vertical FOV had little effect on distance judgments. A two-way repeated measures ANOVA yielded an insignificant main effect of the narrowed FOV $(\mathrm{F}(4,28)=0.278, \mathrm{p}=0.890)$ as well as an insignificant interaction of (FOVs $\mathrm{x}$ Distance) $(F(12,84)=0.902, p=0.548)$. Clearly, the result confirmed the prediction of the SSIP model: distance could be accurately judged by sampling and integrating the ground information along the path from the observer's feet to the target.

\subsection{Exp 6.6. The directional asymmetry in distance judgment}

Since the SSIP hypothesis assumes that the perceived surface portion serves as the reference for representing next surface region, an accurate initial surface representation is thus important for ensuring the correctness of consequent computation. Accordingly, the ground surface can be represented accurately if the computation starts from near space 
where rich depth cues provide for a reliable initial representation. But an erroneous result is likely if the computation initiates from some distant place where an accurate initial surface representation cannot be obtained from inadequate depth cues.

The next two experiments evaluated this hypothesis. Subjects were asked to examine distances from near to far or in the reverse direction. As stated by the SSIP hypothesis, accurate distance judgment was expected in the near-to-far scan condition, but not in the far-to-near scan condition.

\subsubsection{Methods}

Observers. The same eight subjects were tested.

Design. Two horizontal viewing windows were used: $57.7^{\circ}$ horizontally $\mathrm{x}\left(13.6^{\circ}\right.$, $21.1^{\circ}$ ) vertically. The cross-factor design had two independent variables: (1.) the target's distance from the observer $(4,5,6$, and $7 \mathrm{~m})$, and (2.) the scan direction (near-to-far or far-to-near scan). Subjects were tested with 32 trials ( 2 windows $x 4$ distances $x 2$ scan directions $\times 2$ replications). The presentation of experimental trials was blocked by different viewing windows and scan directions, but fully randomized within each block.

Procedure. The procedure was the same as in Exp. 6.5 except that the subject was allowed to examine the path between his feet and the target only once. For the near-to-far scan, the observer first looked down at his feet, and then gradually rotated his head upward and looked towards the target. For the far-to-near scan, the observer rotated his head downward from an initial straight-ahead position and gradually looked towards his feet. When the scan was over, the subject put on the blindfold and started the blindfolded walking. 


\subsubsection{Results}

The directional asymmetry is evidently shown in Figure 6.8(b). Consider the nearto-far scans first. Subjects' estimates of distance in this condition were nearly as accurate as that in the control condition (For the vertical FOV of $13.6^{\circ}: F(1,7)=0.926, p=0.368$ for the main effect of scan, $F(3,21)=0.729, \mathrm{p}=0.546$ for the interaction of (Scan $\mathrm{x}$ Distance); For the vertical FOV of $21.1^{\circ}: F(1,7)=2.372, p=0.167$ for the main effect of scan, $F(3,21)=0.591, p=0.628$ for the interaction of $($ Scan $x$ Distance $))$.

On the other hand, if compared to the static-viewing condition, no overall improvement in distance judgment was found in the far-to-near-scan condition. The main effect of scan was insignificant $\left(F(1,7)=2.648, p=0.148\right.$ for the vertical FOV of $13.6^{\circ}$; $F(1,7)=0.033, p=0.862$ for the vertical FOV of $21.1^{\circ}$ ). But the interaction of (Distance $x$ Scan) was marginally significant because improvement was shown at the target distance of $4 \mathrm{~m}(\mathrm{~F}(3,21))=3.707, \mathrm{p}=0.028$ for the vertical FOV of $13.6^{\circ} ; \mathrm{F}(3,21)=3.058, \mathrm{p}=0.051$ for the vertical FOV of $21.1^{\circ}$ ).

Briefly, accurate distance judgment was obtained from near-to-far scans; while far-to-near scans produce little improvement in subject's judgments and distance remained underestimated. This shows that an accurate ground surface representation can be formed through the integrative process only if the near ground surface is assessed first.

\subsection{Exp 6.7. Distance judgment with two scans}

In the last experiment subjects judged distance with only one scan. So the failure to derive an accurate ground representation from the far-to-near scan might be attributed to the lack of iteration. To examine this possibility, we asked subjects to scan twice when 
judging the distance. A significant improvement in distance judgment was expected, according to the no-iteration explanation.

\subsubsection{Methods}

Observers. The same eight subjects were tested.

Design. Subjects were tested individually with two FOVs: $57.7^{\circ}$ horizontally $\mathrm{x}$ $\left(13.6^{\circ}, 21.1^{\circ}\right)$ vertically. For each FOV, the effect of iteration was examined using a three-way cross-factor design. The independent variables were (1.) the target's distance from the observer $(4,5,6$, and $7 \mathrm{~m}),(2$.$) the scan direction (near-to-far or far-to-near$ scan) and (3.) the iteration of scan (scan once or twice). Subjects were tested with 32 trials (4 distances $\mathrm{x} 2$ scan directions $\mathrm{x} 2$ iterations $\mathrm{x} 2$ replications). The presentation of experimental trials was blocked by combinations of different viewing windows, scan directions and iterations, but fully randomized within each block.

Procedure. The procedure was the same as in the previous experiment except that the subject was asked to scan the path twice in the either near-to-far or far-to-near direction: the subject first scanned the path in the indicated direction once, then put on the blindfold, returned his head to the initial position, and performed the scan again.

\subsubsection{Results}

Figure 6.9 compared subjects' estimates of distance under the scan-once and scantwice conditions. Consider the far-to-near scan first. Similar underestimation of distance was found in the scan-once and scan-twice conditions. No significant improvement was produced by examining the distance one more time in the far-to-near direction (For the vertical FOV of $13.6^{\circ}: F(1,7)=0.120, p=0.740 ; F(3,21)=1.217, p=0.328$; For the vertical FOV of $\left.21.1^{\circ}: F(1,7)=0.720, p=0.424 ; F(3,21)=0.994, p=0.415\right)$. On the other hand, weak 
effects of an additional scan were found in the near-to-far direction. With a vertical FOV of $13.6^{\circ}$, subjects walked a slightly longer distance in the scan-twice condition than in the scan-once condition. But the effect was just marginally significant $(F(1,7)=5.739$, $\mathrm{p}=0.048$ for the main effect of iteration). The interaction of (Iteration $\mathrm{x}$ Distance) was significant $(F(3,21)=5.302, p=0.007)$ for judging distance with the FOV of $21.1^{\circ}$.

The above results rule out the no-iteration explanation. Alternatively, the failure to improve distance judgment with two far-to-near scans plus the weak effects of two near-to-far scans strongly suggest that, to compute an accurate ground representation, the visual system accumulates information only from near to far, not in the reverse direction, and the near ground area is the foundation of this computation.

\subsection{Exp 6.8. Distance judgment in the dark with or without head motion}

This control experiment examined the contribution of the head-motion signal from scan to distance judgment. Since distance underestimation was corrected in the free head-motion condition, accurate distance judgment may be ascribed to the head-motion signal from the motor system, rather than the construction of the ground reference frame. The directional asymmetry in distance judgment may also be explained by the possibility that the motion signal is deficient in one direction but not in the opposite direction. To assess this head-motion hypothesis, subjects were tested in the dark where information about the ground surface was eliminated and distance was judged with or without head motion. If the head-motion signal significantly contributes to the perception of distance, accurate distance judgment and directional asymmetry are expected in the head-motion conditions. 


\subsubsection{Methods}

Observers. The same eight subjects were tested.

Design. The cross-factor design had two independent variables: (1.) the viewing conditions (the static viewing condition, the near-to-far/far-to-near scan conditions, or the free head-motion condition) and (2.) the target's distance from the observer $(2.5,3.75$, 5.0 or $6.25 \mathrm{~m}$ ). Each subject was tested with 32 trials (4 viewing conditions $\mathrm{x} 4$ distances x 2 replications). The experiment was blocked and balanced by different viewing conditions, but was randomized in the presentation of trials within each block.

Procedure. The experiment was conducted in a dark carpeted hallway $(3 \times 11 \mathrm{~m})$. After lights were extinguished, there was no visible reference in the environment and thus the information about the ground surface was eliminated.

At the beginning of each block, the subject was informed the task: to judge the target's location, both distance and height, from a static view, with free head-motion, or with only one scan in either the near-to-far or far-to-near direction. On a given trial, the subject first previewed the target - an internally illuminated Ping-Pong ball (Luminance: $0.16 \mathrm{cdm}^{-2}$, Visual angle subtended: $0.23^{\circ}$ ) that was placed on the floor in test trials or $0.5 \mathrm{~m}$ above the floor in dummy trials. After he had made the estimation, the subject put on the blindfold and informed the experimenter. The experimenter then removed the target. The subject, with eyes blindfolded, walked to where he thought the target was and gestured the perceived height of the target when reaching the destination. The experimenter measured the walked distance and the gestured height and then led the subject back to the starting point. No error feedback was provided to the subject. 


\subsubsection{Results}

Figure 6.10(a) shows the perceived distance and height of the target in four viewing conditions. Noticeably, head motion showed little effect on subjects' performance. Subjects' estimates of distance and height in the dark were almost identical no matter head motion. A two-way repeated measures ANOVA indicated no significant effects of viewing conditions (For distance judgment: $F(3,21)=1.597, p=0.220$ for the main effect of viewing conditions; $F(9,63)=1.218, p=0.300$ for the interaction of (Distance $\mathrm{x}$ Viewing conditions); For height judgment: $\mathrm{F}(3,21)=1.518, \mathrm{p}=0.239$ for the main effect of viewing conditions; $F(9,63)=0.965, p=0.477$ for the interaction of (Distance $\mathrm{x}$ Viewing conditions)). The result thus negates the head-motion hypothesis. Instead, it provides evidence for the "ground theory" (Gibson, 1950, 1979): visual space is determined by the perception of the background surface and veridical space perception is impossible without a visible ground surface. As shown in Figure 6.10(b), without reference to the ground surface, subjects misperceived the target's location, although the angular declination of the target was perceived correctly.

\subsection{Summary}

Findings from these experiments support the SSIP hypothesis. Experiments $6.1 \&$ 6.2 show that accurate distance judgment requires a large-scale representation of an extended ground surface. Experiments 6.3-6.8 address how to build an overall surface representation by integrating local information. Distance judgments in Experiments 6.36.5 reveal the critical role of the near ground information in space perception. Experiments 6.6-6.8 then confirm the directional asymmetry in the surface representation 
process: accurate distance judgment from a correct ground representation is achieved by scanning local patches of the ground surface from near to far, but not in the reverse direction. In summary, converging evidence from the above experiments demonstrates that, for accurate space perception, the visual system relies on an integrative process that forms a global ground surface representation by integrating information on the ground across time and space from near to far. 


\section{CHAPTER 7}

\section{HYPOTHESES ABOUT THE TEXTURE-DISCONTINUITY EFFECT}

This chapter was to evaluate an alternative explanation about the texturediscontinuity effect, the "boundary" hypothesis proposed by Feria et al. (2002, 2003a-b).

\subsection{The boundary hypothesis}

Feria et al. $(2002,2003 a)$ explored the effect of texture discontinuity on distance judgment by using computer-generated displays of simulated surfaces. In their experiments, the background surface was either a ground plane receding in depth or a frontoparallel plane. Discontinuity in surface texture was produced by using different texture patterns, the same texture reversed in contrast, or the same texture with phase shift. Subjects' exocentric distance judgment was measured using a perceptual matching task - to increase or decrease the separation between a pair of poles in the image until it appeared equal to the target distance defined by another pair of poles. Their results found that for all experimental conditions, less distance was judged across the texture boundary than along a uniform texture, no matter the background surface was the ground or a frontal plane. 
Based on these results, Feria et al. argued that distance underestimation might be caused by a general property of all texture discontinuities - the boundary. The idea underlying their "boundary" hypothesis was that "when a surface contains a texture discontinuity, a small area adjacent to the perceived boundary is excluded from judged distances." (p1423, Feria et al., 2003a) They explained the theory as the following:

"Although the boundary mathematically is a line with no extent in the direction across which the judgment is made, perceptually it may occupy a small amount of space. If a small amount of space immediately adjacent to the boundary was not included in the distance computation, the sum of the distance to the boundary and the distance from the boundary to the target would be less than the distance measured in a single step. This proposed explanation, while speculative, can account for the results of the present experiments and for previous results showing a discontinuity effect. It predicts that the discontinuity effect will occur whenever a boundary is present in the interval to be judged, regardless of whether there is a change in texture type. The explanation also predicts that the discontinuity effect will occur whether a surface is extended in depth, frontoparallel, or at any other orientation relative to the observer." (p1423, Feria et al., 2003a)

They further suggested that distance underestimation could be caused by implicit or explicit boundaries, or even line terminations. For example, distance underestimation was found in the "line discontinuity" condition (Experiment 4 of Feria et al. (2003b)), in 
which the target distance was defined by a pair of dots and a line as thin as $0.1 \mathrm{~mm}$ was drawn between the dots.

\subsection{Comments on two hypotheses}

Consistent with the findings that discontinuity in texture results in the underperception of egocentric distance, Feria et al. confirmed the discontinuity effect in exocentric distance perception. But the mechanism underlying distance underestimation remains a controversial issue. The Sequential Surface Integration Process (SSIP) hypothesis argues that underestimation might be attributable to the failure in constructing an accurate surface representation. Alternatively, the "boundary" hypothesis suggests that information exclusion happens so that "a small area adjacent to the perceived boundary is excluded from judged distances". But the theory gives no explanation about why a boundary would make some information overlooked by the visual system.

Notably, the well-known Oppel-Kundet illusion is a counterexample against the "boundary" hypothesis. As shown in Figure 7.1, the space AC is bisected into two parts. The area $A B$ is filled with vertical lines while the area $B C$ is empty. Clearly, $A B$ looks larger than BC. This is in opposition to what expected by the "boundary" hypothesis, which predicts $\mathrm{BC}>\mathrm{AB}$ because areas adjacent to those vertical lines will be excluded from perception.

On the other hand, the SSIP hypothesis explains the illusion by difference in representing the surfaces. Although we discuss the SSIP hypothesis in the context of how to build a ground representation, it is reasonable to apply the theory to accounting for perceiving spatial layout of a frontal plane. Essentially, the theory asserts that 
representing surface is an integrative process that combines information over surface area and time. Such a process is expected in perceiving surface layout of a frontal plane. Hence the Oppel-Kundet illusion is explained by the presence of texture information in $\mathrm{AB}$ but not in $\mathrm{BC}$.

To evaluate these two hypotheses, the discontinuity effect on distance judgment was examined in Exp 7.1 -7.4 on a ground-like surface or a frontal plane.

\subsection{Exp 7.1. The 2D texture-discontinuity effect}

This experiment was to examine the discontinuity effect in a frontal plane. Subjects' exocentric distance judgment was compared when the background surface was continuously or discontinuously textured.

\subsubsection{Methods}

Observers. Eight paid naïve subjects with normal or correct-to-normal vision participated in the experiment.

Design. Two independent variables were used in a cross-factor design. They were: (1). the background patterns (grass/bricks or different colors) and (2). the continuity of the background (continuous or discontinuous). These variables were run within subjects. For each combination of these variables, the target distance (300 pixels or $9.8 \mathrm{~cm}$ on the monitor) was measured with 8 replications. In addition, 8 dummy trials, in which the stimulus distance was randomly set to a value between 150 and 550 pixels, were tested in each condition. Thus a total of 64 trails were tested and the presentation of test or dummy trials was fully randomized so that subject could not tell them apart. 
Experimental Setting and Stimuli. Subjects sat at a table on which a 17" color monitor was placed at eye level. The stimulus was displayed on the monitor with a resolution of $1024 \times 768$ and a refresh rate of $100 \mathrm{~Hz}$. A chinrest was used to maintain a constant viewing distance of $38 \mathrm{~cm}$. The target distance of 300 pixels subtended a $14.6^{\circ}$ (vertical) of visual angle on the screen.

Procedure. Subjects were tested individually in a dimly lit room. After reading the instruction, subjects completed sixteen practice trials to familiarize them with the procedure.

Each trial began with a warning tone and the stimulus was presented after 1 second. An example of the stimuli used in this experiment is shown in Figure 7.2(a). The subject saw two pairs of markers in the picture and the target distance was the separation between the vertical pair of markers. The subject's task was to compare the separations defined by two pairs of markers and then adjust the horizontal separation by pressing the arrow keys on the keyboard until both distances seemed equal. When matched, the subject finished the trial by pressing the Enter key. Then a random-dots mask would be displayed for a duration of $500 \sim 1500 \mathrm{~ms}$. And the next trial started after the masking. On average, each trail took about 30 seconds. No error feedback was provided in the experiment.

The experiment computer recorded the judged distance. It was measured in units of pixels, where 100-pixels is equivalent to $3.25 \mathrm{~cm}$.

\subsubsection{Results}

Figure 7.2(b) shows the mean judged distances averaged across 8 subjects. In all conditions the judged distances (341.4 357.4 pixels) were larger than the target distance 
(300 pixels). This might be ascribed to the vertical-horizontal illusion, since the target distance was defined by a vertical separation while the matching was performed in the horizontal direction.

Importantly, the discontinuity effect was evident in the figure. Compared to the continuous-background conditions, subjects judged a shorter distance in the discontinuous-background conditions (mean judged distance: 355.4 vs. 342.8 pixels). A two-way repeated measures ANOVA (Background patterns $x$ Continuity) found a significant main effect of Continuity $(F(1,7)=11.097, p<0.02)$. Thus, the experiment replicated Feria et al.'s finding (Feria et al., 2003a).

\subsection{Exp 7.2. Influence of perspective information on the discontinuity effect}

Having confirmed the texture-discontinuity effect in the frontal plane, we next went on to examine the "boundary" hypothesis. We first investigated what information would contribute to the discontinuity effect. The "boundary" hypothesis asserted that boundaries in the display determine the effect, regardless of texture information. To test this, subjects' distance judgments were measured with reference to different background images that displayed a frontal plane or a ground-like surface (Figure 7.3(a)). Also, a matching-on-the-screen instruction was used, which asked subjects to compare distances on the screen, not in depth. Accordingly, no influence of the background image should be expected by the "boundary" hypothesis when similar boundaries were present in the background images.

\subsubsection{Methods}

Observers. The same eight subjects were tested. 
Design. The cross-factor design was the same as in the previous experiment, except that two independent variables were: (1). the presence of perspective information (2D or $3 \mathrm{D}$ background image) and (2). the continuity of the background (homogeneous or discontinuous).

Procedure. The procedure was the same as in the previous experiment. Again, it should be emphasized that this experiment used the same matching-on-the-screen instruction as in the previous experiment. That is, subjects were clearly instructed to compare distances on the computer screen, regardless of whether the background image displayed a ground or a frontal plane.

\subsubsection{Results}

Figure 7.3(b) shows the mean judged distances averaged across 8 subjects. The discontinuity effect was evident in the results. A two-way repeated measures ANOVA (Perspective information $\mathrm{x}$ Background continuity) found a significant main effect of Continuity $(\mathrm{F}(1,7)=25.987, \mathrm{p}<0.001)$.

More important, subjects' distance judgment was significantly influenced by perspective information, although the matching-on-the-screen instruction encouraged subjects to overlook depth information in the background image and compare distances directly on the screen. The mean judged distance was longer in the presence of perspective information than without it (391.1 vs. 350.8 pixels). The ANOVA confirmed this with a significant main effect of Perspective-Information $(F(1,7)=22.077, p<0.005)$. This result agrees with Gibson's idea that the perception of distance or depth is reducible to the perception of the background surface on which objects rest. Although a separation of 300 pixels subtended a constant visual angle in the retinal image, the judged distance 
varied with the perception of the background surface. When a ground-like background was displayed by perspective information, subject judged a larger distance in this condition than in the frontal-background condition.

The texture discontinuity effect was also significantly influenced by perspective information. As shown in the left column of Figure 7.3(b), distance underestimation was larger in the presence of perspective information than in the absence of it ( 28.4 vs. 13.2 pixels). To compare the effect, we calculated it as a percentage of the mean judged distance, i.e. Effect $=100 \% \times\left(D_{\text {Continuous }}-D_{\text {Discontinuous }}\right) /\left(\left(D_{\text {Continuous }}+D_{\text {Discontinuous }}\right) / 2\right)$ (Equation 7.1) where D denotes the judged distance. The discontinuity effect was $7.10 \%$ and $3.75 \%$ in the with-perspective and without-perspective conditions, respectively. That is, the effect was almost doubled by the perspective information in the background. Also, the ANOVA found a significant interaction of (Perspective-Information $x$ Continuity) $(F(1,7)=8.562, p<0.025)$. Clearly, the discontinuity effect was influenced the perception of the background surface.

Briefly, the results suggest that distance judgment depends on the perception of the background surface. The discontinuity effect thus cannot be solely accounted for by some qualities in the retinal image like boundaries. Instead, it might be attributed to some process of representing the background surface, as the SSIP hypothesis has suggested.

\subsection{Exp 7.3. Distance judgment across different number of boundaries}

This experiment was to test the notion that the discontinuity effect was because "a small area adjacent to the perceived boundary is excluded from judged distances". Here distance was judged in a frontal plane but across different numbers of boundaries. As 
stated by the "boundary" hypothesis, distance underestimation would increase with number of boundaries because more and more areas would be excluded from the perception.

\subsubsection{Methods}

Observers. Eleven naïve subjects were tested.

Design. Subjects' distance judgment was measured with 8 repeats in four conditions: the control condition, 2-boudaries condition in which a strip of different color was placed between the target separation, 4-boudaries condition with two strips between the target separation, and 6-boudaries condition with three strips between the target separation. Figure 7.4(a) shows the example stimuli of the control, 2-boundairies and 6boudaires conditions. A total of 64 trails were tested with 32 dummy trials. The presentation of these trials was fully randomized.

Procedure. The procedure was the same as in the previous experiment.

\subsubsection{Results}

The left column of Figure 7.4(b) shows the mean judged distance for individual subjects in four conditions, while the right column shows the averaged response for all subjects. Clearly, the pattern in subjects' responses disagrees with the prediction from the "boundary" hypothesis. Of three discontinuous conditions, the shortest distance was judged in the 2-boudaries condition. When more stripes were placed, distance underestimation did not build up, but reduced. Especially, the judged distance was nearly identical in the control and 6-boudaries conditions (mean judged distance: 380.8 vs. 380.9 in the control and 6-boudaries conditions; paired t-test: $t(10)=-0.002, p=0.998$ ). The discontinuity effect was calculated using Equation 7.1 for three discontinuous 
conditions. It was $7.70 \%, 3.71 \%$ and $0.01 \%$ in $2-$, 4-, and 6-boudaries conditions, respectively. A one-way repeated measures ANOVA found a significant effect of Number-of-Boundaries $(F(3,30)=12.842, \mathrm{p}<0.001)$.

As an alternative, the result could be accounted for by the surface integration hypothesis. According to the theory, it is errors in surface representation, not the boundaries, that cause underestimation in distance judgment. Take Figure 7.5(a) as the example. The same separation defined by pairs of dots appears shorter in the right image than in the left image. Also, it is noticeable that the grey area in the right image looks smaller than the rectangle in the left image, although both are actually same. This might be explained as in Figure $7.5(\mathrm{~b})$. Due to the foreground/background segmentation, the gray area is perceived as another surface that is somewhat above the white background. Accordingly, it is judged smaller than the rectangle on the background, although they subtend the same visual angle in the image. Moreover, the perceived occlusion causes information loss in representing the background surface, because occlusion only signals relative depth and gives no information about the size of hidden area. Nor can amodal surface completion form an accurate surface representation without reference to additional information such as texture, or binocular information. As a result, distance is underestimated when judged across the gray area. On the other hand, when a number of strips are displayed like in Figure 7.4(a), the regularity and repeatability in the strips' placement depict them as texture on the surface. And so the discontinuity effect is reduced because texture information is useful for the visual system to perceive surface layout. 
In conclusion, the results disagree with the "boundary" hypothesis, but provide support for the surface integration hypothesis.

\subsection{Exp 7.4. Influence of amount of occlusion on the occlusion effect}

The occlusion effect in distance judgment was examined here to contrast two hypotheses. As shown in Section 5.4, distance would be underestimated when a continuous ground was partially occluded (For illustration, see Figure 5.6). Thus, the relative depth between two targets would be underestimated when a wall was placed between them. In this experiment we investigated how distance underestimation was influenced by the extent of the occlusion. As shown in Figure 7.6(a), the wall's size was manipulated: it might extend over the whole width of the image (full-size-wall-condition) or only a part of the image (half- or quarter-size-wall-condition). According to the surface integration hypothesis, as the wall shrink, less distance underestimation would result from the destruction of the ground surface due to smaller occlusion. On the other hand, the "boundary" theory expected similar distance underestimation across all occlusion conditions because same boundaries were placed between the targets.

\subsubsection{Methods}

Observers. The same eleven subjects were tested as in the previous experiment.

Design. Subjects' distance judgment was measured with 8 repeats in four conditions: the control condition, full-size-wall-condition, half-size-wall-condition, and quarter-size-wall-condition. Figure 7.6(a) shows the example stimuli of the control, fullsize-wall and quarter-size-wall conditions. A total of 64 trails, half test trials and half dummy trials, were tested. The presentation of these trials was fully randomized. 
Procedure. The procedure was the same as in the previous experiment. Importantly, this experiment used the same matching-on-the-screen instruction as in the previous experiments. Subjects were instructed to compare horizontal and vertical separations on the computer screen, not in depth.

\subsubsection{Results}

Figure 7.5(b) shows subjects' responses in four conditions. Undoubtedly, the pattern in subjects' responses is consistent with the prediction from the surface integration hypothesis. Compared to the control condition, distance was underestimated most in the full-size-wall-condition $(9.33 \%$ of the mean judged distance, calculated using Equation 7.1), and the underestimation was reduced almost by half in the half-size-wallcondition (5.34\%) and quarter-size-wall-condition (5.09\%). A one-way repeated measures ANOVA found a significant effect of Occlusion-Extent $(F(3,30)=11.728$, $\mathrm{p}<0.001)$. Briefly, the result provides direct support for the surface integration hypothesis.

\subsection{Summary}

To sum up, consistent with Feria et al.'s report (2002, 2003a-b), four experiments had confirmed the texture discontinuity effect on exocentric distance judgment. However, evidence from Experiment 7.2-7.4 gives little support to the "boundary" hypothesis that "when a surface contains a texture discontinuity, a small area adjacent to the perceived boundary is excluded from judged distances." (p1423, Feria et al., 2003a). Especially, Experiment 7.3 found that distance underestimation reduced with more boundaries, not increased as predicted by the "boundary" hypothesis. Instead, the results provide direct 
support for the surface integration hypothesis. Experiments $7.2 \& 7.4$ clearly show that distance perception as well as the discontinuity effect relies on the perception of the background surface. In Experiments 7.4, distance underestimation reduced with smaller occlusion as larger ground surface became visible. These results thus reinforce the idea underlying the SSIP hypothesis that the visual system relies on an integrative process that combines information over surface and time to build an accurate surface representation and hence perceived surface layout correctly. Therefore, the perception of a frontal plane might be considered as a special case of representing 3D surfaces, which is processed by the same surface integration process. 


\section{CHAPTER 8}

\section{CONCLUSIONS}

\subsection{Summary of results}

This investigation considered the problem of how people make judgments about distance within action space (about $2 \sim 30 \mathrm{~m}$ ). Two general problems are addressed by the experiments: what information can be used to judge distance and how is it processed?

For the "what" question, the results are summarized in the following:

1. Angular declination is a strong cue for distance. Experiments reported in Chapter 2 show that the visual system is able to accurately perceive the information regarding the target's angular declination even in the cue-reduced environment. Accordingly, the target is visually located on the projection line from the observer's eyes to it (direction constancy).

2. The experiments in Chapter $3 \& 5$ show that the ground might be the reference for coding objects' locations and the ground texture is a significant source of information for representing the surface in the perceptual world. A continuous ground texture is found to be essential for veridical distance judgment.

3. Of multiple textural cues, Chapter 3 shows that linear perspective is a powerful cue for representing the ground surface and judging distance but texture compression little effective. 
For the "how" question, the experiments found:

1. The representation of an extended ground surface that stretches from the observer to the target is necessary for veridical space perception. We argue that such a representation is formed by an integrative process that integrates local information over space and time.

2. The Sequential Surface Integration Process (SSIP) hypothesis is proposed to understand the process of representing the ground. It claims that an accurate representation is formed by sampling and combining information across time and space and reliable near depth cues are used to calibrate the overall representation. Confirming this, the experiments in Chapter 3,5\&6 found that the near ground area has a significant role in space perception. Without access to it, distance would be underestimated when an observer's view was restricted to the local ground area about the target.

3. Another evidence for the SSIP hypothesis is the directional asymmetry in representing the ground. An accurate representation and hence accurate distance judgment is achieved only by scanning local patches of the ground surface from near to far, but not in the reverse direction.

\subsection{Concluding remarks}

So far, we have got answers for some questions. The results demonstrate that the visual system is able to construct an accurate representation for the ground surface and hence judge distance on it by using the angular declination information. But the exact mechanism underlying space vision has yet to be determined. Although the current study has shown that distance judgment is influenced by information about angular declination, 
the registered eye height, and the ground representation, it is unclear if the visual system does do the trigonometry. Also it remains mysterious how visual space is represented in the brain and how integration of available information is performed by the nervous system. In future study it is worth our while to further investigate integrating over an extended spatial region. Through this line of investigation, the ground-based space perception may begin to be understood.

In addition, space is usually underperceived in virtual environments. Previous research has attacked this problem from different approaches, such as limited field of view of head-mounted displays or incorrect binocular information in the display. Here this work suggests a different way to consider the problem. The compressed space and underestimation of absolute distance in VR might be due to an incorrect representation of the ground surface. Thus it would be necessary to identify what is important to perceive the ground surface correctly in virtual environments. Following this approach, the problem might be solved. 


\section{REFERENCES}

Bhalla, M., \& Proffitt, D. R. (1999). Visual-Motor recalibration in geographical slant perception. Journal of Experimental Psychology: Human Perception \& Performance. 25(4), 1076-1096.

Braunstein, M.L. (1968). Motion and texture as sources of slant information. Journal of experimental psychology, 78, 247-253.

Clark W.C., Smith, A. H., \& Rabe, Ausmac(1956). The interaction of surface texture , outline gradient, and ground in the perception of slant. Canadian Journal of Psychology. 10, 1-8.

Cook, M. (1978). The judgment of distance on a planar surface. Perception \& Psychophysics. 23, 85-90.

Creem, S. H. \& Proffitt, D. R. (1998). Defining the cortical visual systems: "What", "where", and "how". Acta Psychologica, 107, 43-68.

Cutting, J.E., \& Millard, R.T. (1984). Three Gradients and the Perception of Flat and Curved Surfaces, Journal of Experimental Psychology: General, 113(2): 198-216.

Cutting, J. E., \& Vishton, P. M. (1995). Perceiving layout and knowing distance: The integration, relative potency and contextual use of different information about depth. In Epstein W. \& Rogers, S. (Eds.) Perception of Space and Motion, Academic Press, New York, 69-117.

Da Silva, J. A. (1982a). Scaling apparent distance in a large open field: presence of a standard does not increase the exponent of the power function. Perceptual and Motor Skills, 55, 267-274.

Da Silva, J. A. (1982b). Scales for subjective distance in a large open field from the fractionation method: Effects of type of judgment and distance range. Perceptual and Motor Skills, 55, 283-288.

Dolezal, H. (1982). Living in a world transformed. Perceptual and performtory adaptation to visual distortion. Academic Press, New York. 
Eby, D.W. \& Loomis, J.M. (1987). A study of visually directed throwing in the presence of multiple distance cues. Perception and Psychophysics, 41, 308-312.

Elliot, D. (1986). Continuous visual information may be important after all: A failure to replicate Thomson (1983). Journal of Experimental Psychology: Human Perception and Performance, 12, 388-391.

Elliot, D. (1987). The influence of walking speed and prior practice on locomotor distance estimation. Journal of Motor Behavior, 19, 476-485.

Flock, H.R. (1964). Some conditions sufficient for accurate monocular perceptions of moving surface slants. Journal of Experimental Psychology, 67, 560-572.

Feria, Cary S., Braunstein, Myron L., \& Andersen, George J. (2003a). Judging distance across texture discontinuities. Perception. 32, $1423-1440$

Feria, C. S., Braunstein, M. L., \& Andersen, G. J. (2003b). Judging distance across discontinuities in the frontal plane [Abstract]. Journal of Vision, 3(9), 645.

Feria, C. S., \& Braunstein, M. L. (2002). Judging distance without a continuously textured ground surface [Abstract]. Journal of Vision, 2(7), 719a.

Foley, J.M. (1977). Effect of distance information and range on two indices of visually perceived distance. Perception, 6, 449-460.

Gilinsky, A.S. (1951). Perceived size and distance in visual space. Psychological Review. $58,460-482$.

Gibson J. J., (1946). Perception of distance and space in the open air. In Gibson, J.J. (Eds), Motion Picture Testing and Research. AAF program Report \#7.

Gibson, J. J. (1950a). The Perception of Visual Surfaces, American Journal of Psychology 58, 367-384.

Gibson, J. J. (1950b). The Perception Of The Visual World. Boston: The Riverside Press.

Gibson, J. J., (1979). The Ecological Approach to Visual Perception, Boston: Houghton Mifflin.

Gibson, J.J. \& Cornsweet, J. (1952). The perceived slant of visual surfaces - optical and geographical. Journal of Experimental Psychology, 44, 11-15.

Gillam, B. (1995). The perception of spatial layout. In Epstein. W. \& Rogers, S. (Eds) Handbook of Perception and Cognition: Perception of Space and Motion (chap. 2., p23-67). San Diego, CA: Academy Press. 
Gogel W.C. (1965). Equidistance tendency and its consequences. Psychological Bulletin, $64,153-163$.

Gogel, W.C. (1977). The metric of visual space. In W. Epstein (Ed.), Stability and constancy in visual perception: Mechanisms and processes (p.129-182). New York: Wiley.

Gogel, W.C. (1990). A theory of phenomenal geometry and its applications, Perception \& Psychophysics, 48, 105-123.

Goldstein, BG (1996). Sensation \& Perception (4th ed.). Brooks/Cole Publishing Company.

Hagen, M. A., Jones, R. K., and Reed, E. S. (1978). On a neglected variable in theories of pictorial perception: Truncation of the visual field, Perception \& Psychophysics, 23(4): 326- 330.

Hagen, M.A. \& Teghtsoonian, M. (1981). The effects of binocular and motion-generated information on the perception of depth and height. Perception \& Psychophysics. $30,257-265$.

Harway, N.L. (1963). Judgment of distance in children and adults. Journal of experimental psychology, 65, 385-390.

He Z.J., Wu B \& Ooi T.L. (2000). Non-vertical distance perception due to false perspectives. ARVO Annual Meeting, May 2000.

He, Z. J., Wu, B., Ooi, T. L., Yarbrough, G. \& Wu, J. (2004) Judging egocentric distance on the ground: occlusion and surface integration" Perception, (in press).

Knill, D. C. (1992). The perception of surface contours and surface shape: from computation to psychophysics. Journal of the Optical Society of America A, 9 (9), 1449-1464.

Knill, D. C. (1998a). Surface orientation from texture: ideal observers, generic observers and the information content of texture cues. Vision Research, 38, 1655-1682.

Knill, D. C. (1998b). Discrimination of planar surface slant from texture: human and ideal observers compared. Vision Research, 38, 1683-1711.

Knill, D. C. (1998c). Ideal observer perturbation analysis reveals human strategies for inferring surface orientation from texture, Vision Research, 38, 2635-2656.

Kunnapas, T. (1968). Distance perception as a function of available visual cues. Journal of Experimental Psychology, 77(4), 523-529. 
Levin, C. A. \& Haber, R. N. (1993). Visual angle as a determinant of perceived interobject distance. Perception \& Psychophysics, 54, 250-259.

Levin, C. A. \& Rosinski, R.R. (1976). Distance perception under binocular and monocular viewing conditions. Perception \& Psychophysics, 19, 460-465.

Loomis, J.M. (1999). Perspective Information and the Perception of Location and Shape. Fifteenth Annual Meeting International Society for Psychophysics, Tempe, Arizona.

Loomis, J. M., Da Silva, J.A., Fujita, N., \& Fukusima, S. S. (1992). Visual space perception and visually directed action. Journal of Experimental Psychology: Human Perception and Performance, 18, 906-921.

Loomis, J. M., Da Silva, J. A., Philbeck, J. W. \& Fuksima, S. S., (1996). Visual Perception of the Location and Distance, Current Directions in Psychological Science, 5(3), 72-77.

Loomis, J. M. \& Philbeck, J. W. (1999). Is the anisotropy of perceived 3-D shape invariant across scale? Perception \& Psychophysics, 61, 397-402

Mark, L. S. (1987). Eye-height scaled information about affordances: a study of sitting and stair climbing. Journal of Experimental Psychology: Human perception \& Performance. 13, 360-370.

Milner, A. D. \& Goodale, M. A. (1995). The visual brain in action. New York: Oxford University Press.

Lumsden, E.T (1980). Problems of magnification and minification: An example of the distortions of distance, slant, shape and velocity. In Hagen, M.A.(Eds) The Perception of Picture. Academic Press, New York, NY.

Marr, D. (1982). Vision. San Francisco: Freeman.

Nagata, S. (1991). How to reinforce perception of depth in single two-dimensional pictures. In S. R. Ellis, M. K. Kaiser, \& A. C. Grunwald(Eds.), Pictorial communication in virtual and real environments (pp. 527-545). London: Taylor \& Francis.

Newman, C.V. (1971). Influence of visual texture density gradients on relative distance judgements. Quarterly Journal Of Experimental Psychology 23, 225-233.

Newman, C.V., Whinham E.A., \& Macrae AW (1973) Influence Of Texture On Judgments Of Slant And Relative Distance In A Picture With Suggested Depth. Perception \& Psychophysics 14, 280-284. 
Ooi, T.L., He, Z.J., \& Wu, B. (2002). Delineating the perceived ground surface from a direction constancy rule. VSS Annual Meeting, May 2002.

Ooi, T.L., Wu, B. \& He, Z.J. (2001). Distance determined by the angular declination below the horizon. Nature, 414, 197 - 200.

Philbeck, J. W. \& Loomis, J. M. (1997). Comparison of two indicators of perceived egocentric distance under full-cue and reduced-cue conditions. Journal of Experimental Psychology: Human perception \& Performance. 23, 72-85

Phillips, R.J. (1970). Stationary visual texture and the estimation of slant angle. Quarterly Journal of Experimental Psychology, 22, 389-397.

Pizlo, Z. (2001). Perception viewed as an inverse problem. Vision research, 41, 31453161 .

Proffitt, D. R. Bhalla, M., Gossweiler, R., \& Midgett, J. (1995). Perceiving geographical slant. Psychological Bulletin and Review. 2, 409-428.

Proffitt, D. R., Creem, S. H. \& Zosh, W. (2001). Seeing mountains in mole hills: Geographical slant perception. Psychological Science, 12, 418-423.

Purdy, W.C. (1960). The hypothesis of psychophysical correspondence in space perception. Ph.D. Thesis, Cornell University.

Purdy, J. \& Gibson, E.J. (1955). Distance judgment by the method of fraction. Journal of Experimental Psychology, 6, 374-381.

Rieser, J.J., Ashmead, D.H., Talor, C.R. \& Youngquist G.A. (1990). Visual perception and the guidance of locomotion without vision to previously seen targets. Perception 19, 675-689.

Sedgwick, H. A. (1986). Space perception. In K. Boff. L. Kaufman, \& J. Thomas (Eds.), Handbook of perception and human performance (chap.21, pp. 1-57). New York: Wiley.

Sinai, M. J., Ooi T. L., He J. Z., (1998). Terrain influences the accurate judgment of distance, Nature, 395, 497-500.

Smith, O.W. (1958). Judgments of size and distance in photographs. American Journal of Psychology, $71,529-538$.

Stevens, K.A. (1979). Representing and analyzing surface orientation. In Winston P.H. \& Brown, R.H. (eds.) Artificial Intelligence: An MIT Perspective, (p104-125). Cambridge: MIT Press. 
Stevens, K.A. (1981). The information content of texture gradients. Biological Cybernetics 42, 95-105.

Stevens, K.A. (1983). Surface tilt (the direction of surface slant): a neglected psychophysical variable. Perception and Psychophysics, 33, 241-250.

Stevens, K.A. (1984). On gradients and texture "gradients". Commentary on: Cutting \& Millard 1984. Three gradients and the perception of flat and curved surfaces. Journal of Experimental Psychology: General 113, 217-220.

Stoper A.E. \& Cohen, M.M. (1986). Judgments of eye level in light and darkness. Perception and Psychophysics 40. 311-316.

Stoper A.E. \& Cohen, M.M. (1991). Optical, gravitational and kinesthetic determinants of judged eye level. In Ellis, S.R. Kaiser, M.K. \& Grunwald, A.C.(Eds). Pictorical communication in virtual and real environments. Taylor \& Francis Ltd, Bristol, PA.

Teghtsoonian R. \& Teghtsoonian M. (1970). Scaling apparent distance in a natural outdoor setting. Psychonomic Science, 21(4), 215-216.

Thompson, J.A. (1983). Is continuous visual monitoring necessary in visual guided locomotion? Journal of Experimental Psychology: Human perception \& Performance. 9: 427-443.

Todd, J.T., \& Akerstrom, R.A. (1987). Perception of Three-Dimensional Form From Patterns of Optical Texture. Journal of Experimental Psychology: Human perception \& Performance. 13(2):242-255.

Toye, R. C. (1986). The effect of viewing position on the perceived layout of space. Perception\& Psychophysics, 40, 85-92.Wagner, M. (1985). The metric of visual space. Perception \& Psychophysics, 38, 483-495.

Wagner, M. (1985). The metric of visual space. Perception and Psychophysics, 38(6), 483-495.

Wallach, H. \& O'Leary, A. (1982). Slope of Regard as a Distance Cue. Perception \& Psychophysics, 31, 145-148.

Warren, W. H. \& Whang, S. (1987). Visual guidance of walking through apertures: bodyscaled information for affordances. Journal of Experimental Psychology: Human perception \& Performance. 13, 371-383.

Werner, E. B. (1991). Manual of visual fields. New York: Churchill Livingstone. 
Wiest, W. M. \& Bell, B. (1985). Stevens's exponent for psychophysical scaling of perceived, remembered, and inferred distance. Psychological Bulletin, 98(3), 457470.

Wohlwill J. F. (1963). Overconstancy in distance perception as a function of the texture of the stimulus filed and other variables. Perceptual and Motor Skills, 17, 831846.

Wohlwill J. F. (1965). Texture of the stimulus field and age as variables in the perception of relative distance in photographic slides. Journal of Experimental Child Psychology, 2, 163-177.

Wu, B., He, Z.J. \& Ooi, T.L.(2001). Judging absolute distance by relying on linear perspective and texture density cues. VSS Annual Meeting, May 2001.

Wu B., He Z.J. \& Ooi T.L. (2002). A ground surface based space perception in the virtual environment. VSS Annual Meeting, May 2002.

Wu, B., Ooi, T. L. and He, Z. J. (2004). Perceiving distance accurately by a directional process of integrating ground information. Nature, 428, 73-77

Yarbrough, G.L., Wu, B., Wu, J. He, Z.J. \& Ooi, T.L.(2002). Judgments of object location behind an obstacle depend on the particular information selected.. VSS Annual Meeting, May 2002.

Yonas, A. Granrud, C.E., Afterberry, M.E. \& Hanson, B.L. (1986). Infants' distance perception from linear perspective and texture gradients. Infant Behavior and Development, 9, 247-256. 


\section{FIGURES}




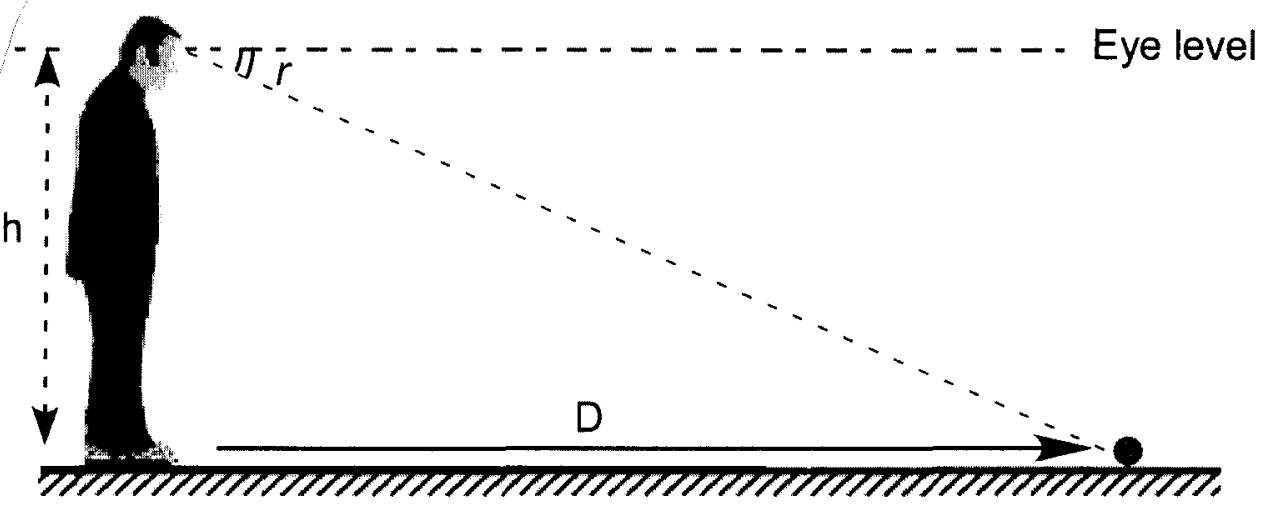

(a)

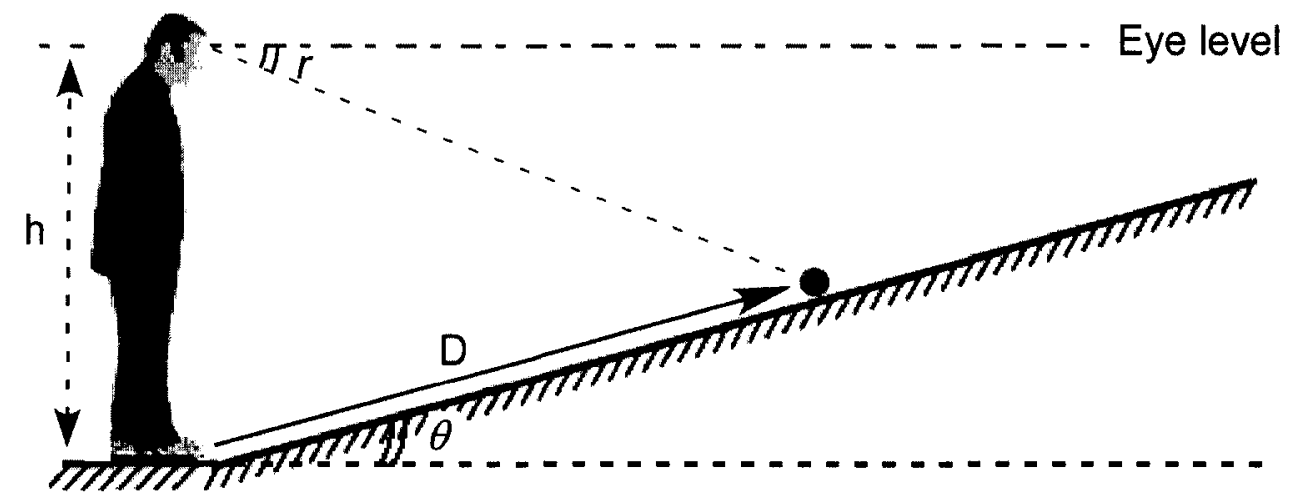

(b)

Figure 1.1. (a) Distance on a level ground can be computed by dividing the observer's eye height by the tangent of angular declination: $D=H / \tan (r)$.

(b) Distance on a slope can be computed by $D=H^{*} \cos (r) / \sin (r+\theta)$. 


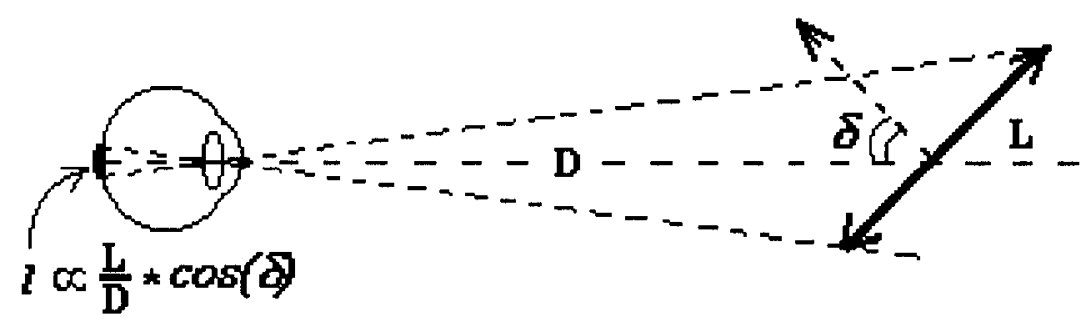

(a)

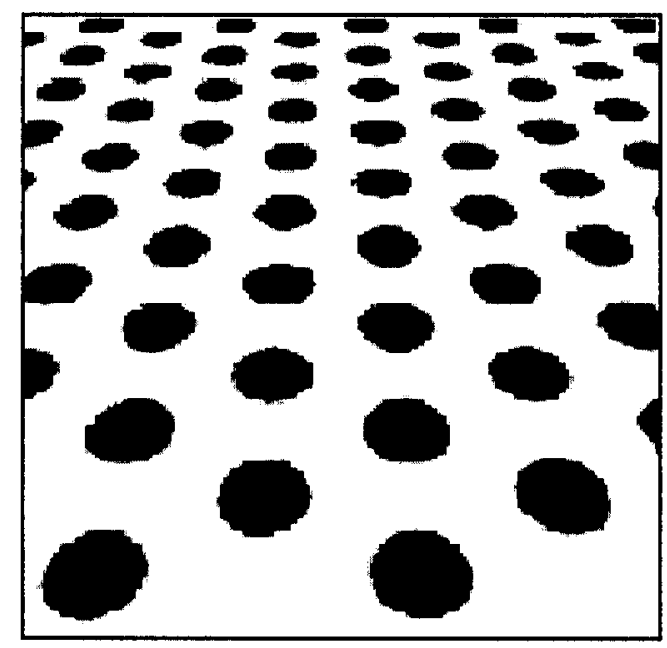

(b)

Figure 1.2. (a). An object's appearance is distorted by perspective projection in two ways: by scaling its size (the $1 / D$ component) and by foreshortening its $\begin{array}{ll}\text { shape (the } \cos (\delta) \text { component). } & \text { (b). Perspective projection distorts the }\end{array}$ appearance of individual texture elements as well as the global structure of texture pattern. Circular disks look more elliptical and smaller with increasing distance. Owing to decreasing spacing, lines of disks eventually merge. All these changes may serve as depth cues. 


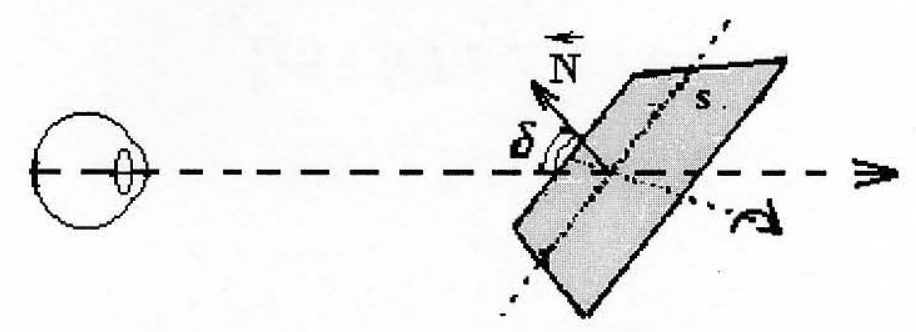

(a)

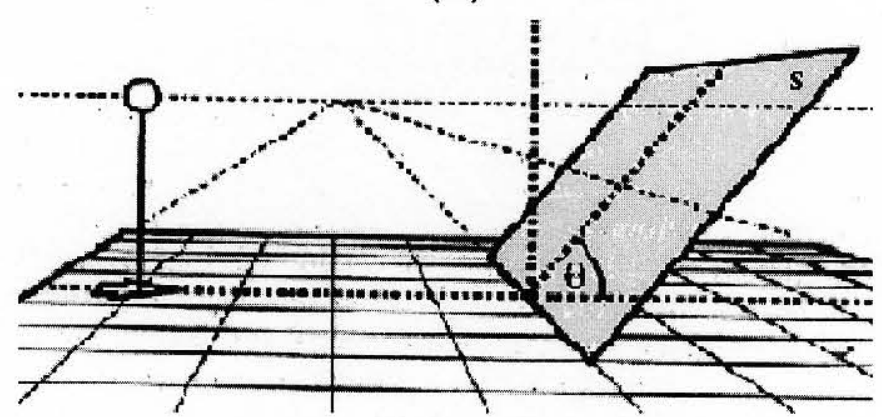

(b)

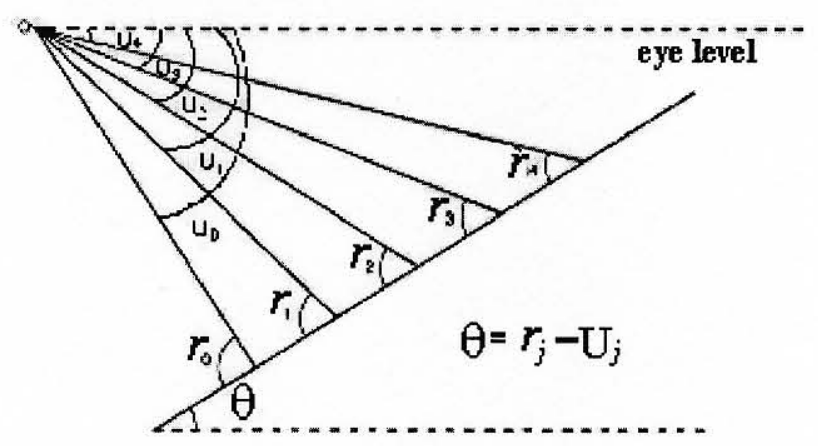

(c)

Figure 1.3. Surface orientation can be defined using either a viewer-centered coordinate system or a world coordinate system. (a). Optical surface slant $(\delta$, $0^{\circ} \leq \delta \leq 90^{\circ}$ ) is defined with reference to the line of sight. (b). Geographical surface slant $(\theta)$ is defined with reference to the horizontal plane in the environment.

(c). The relation between the optic and geographical slant: $\theta=r-U=\left(90^{\circ}-\delta\right)-U$. 
Target: On the ground

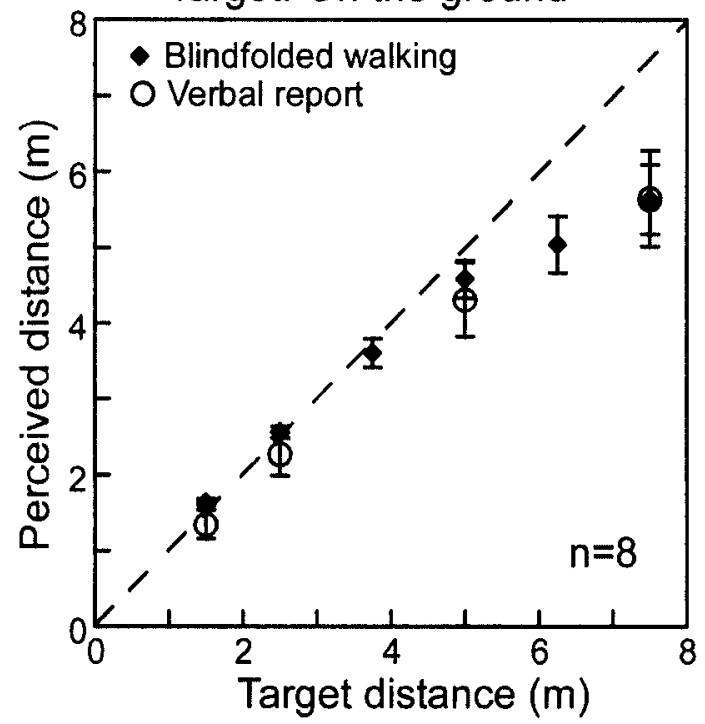

Target: $0.5 \mathrm{~m}$ below the eye level

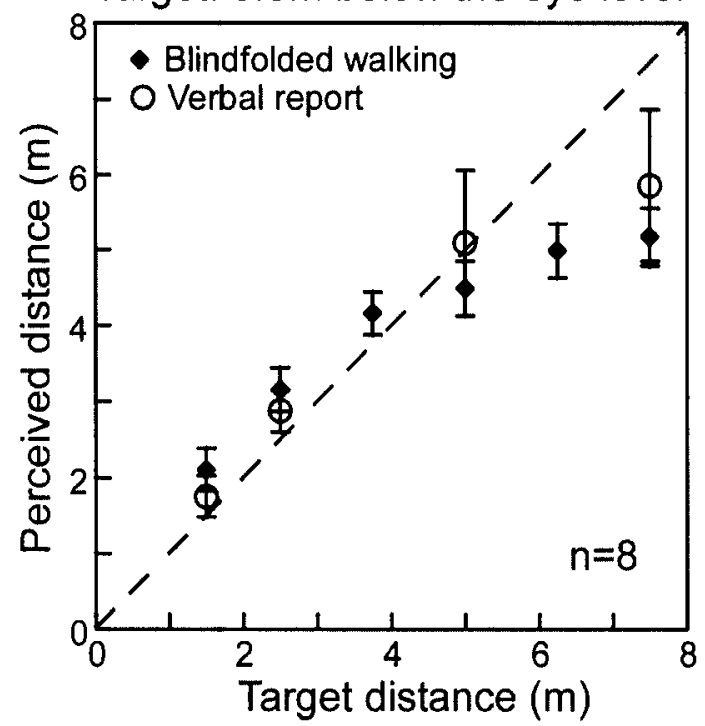

Target: $0.5 \mathrm{~m}$ above the ground

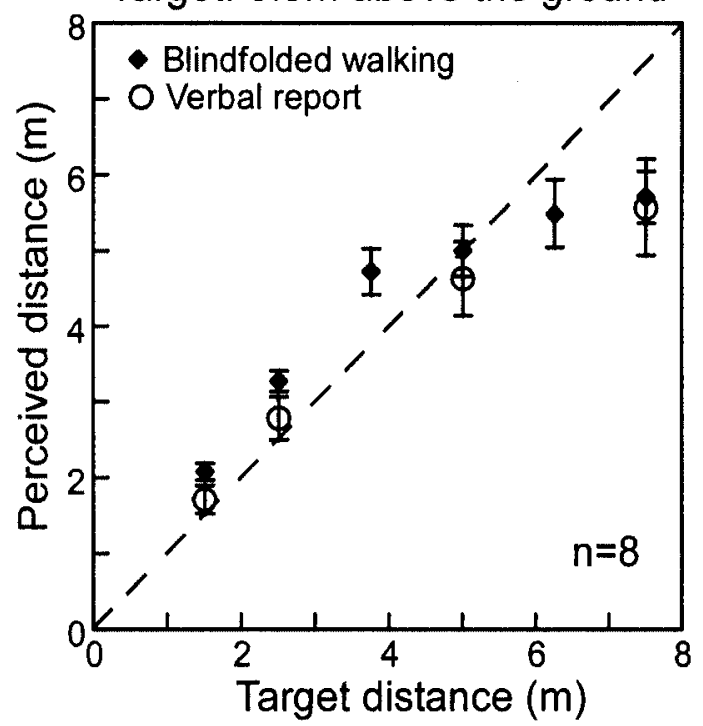

Target: At the eye level

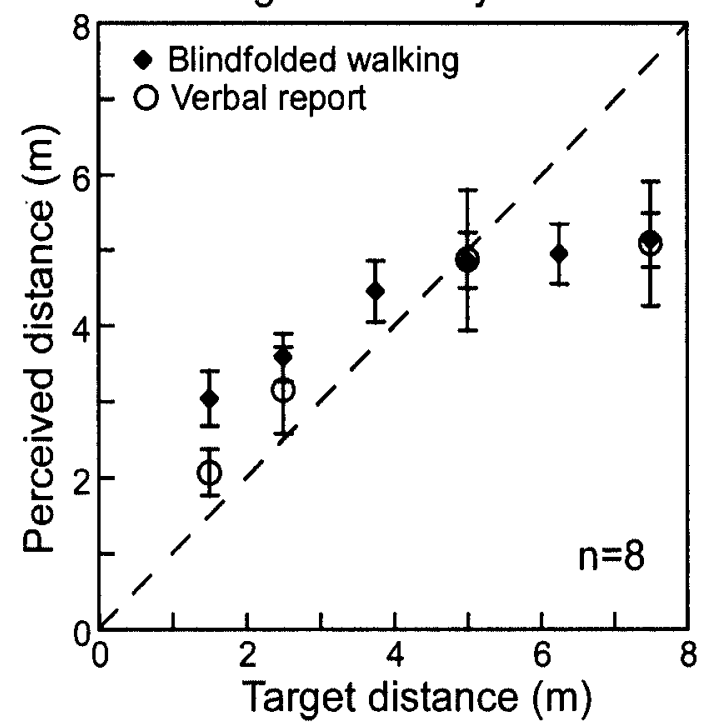

Figure 2.1. Distance judgment in the dark. The mean perceived distance is plotted as a function of the target distance for four target heights. Error bars represent 1 SE. 


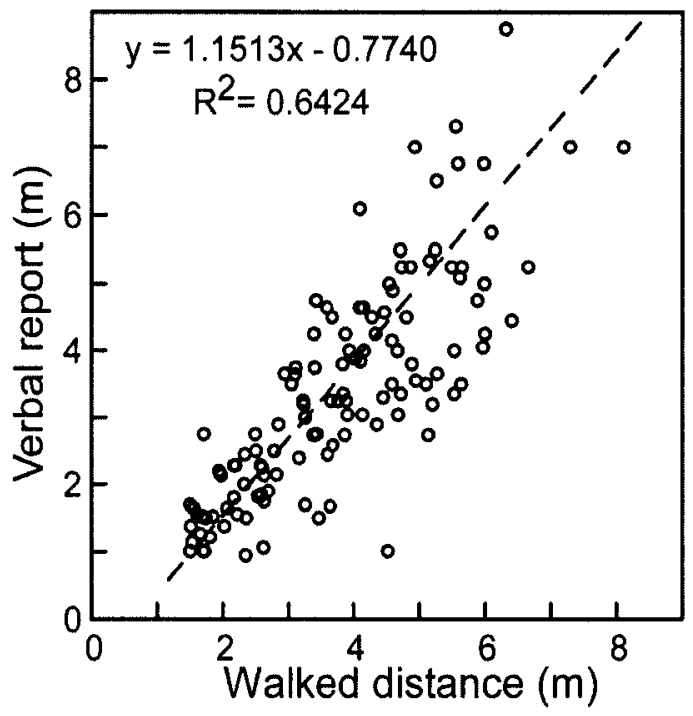

Figure 2.2. Verbal report as a function of walked distance. The regression line shows the linear mapping between two types of responses. 
Target: On the ground
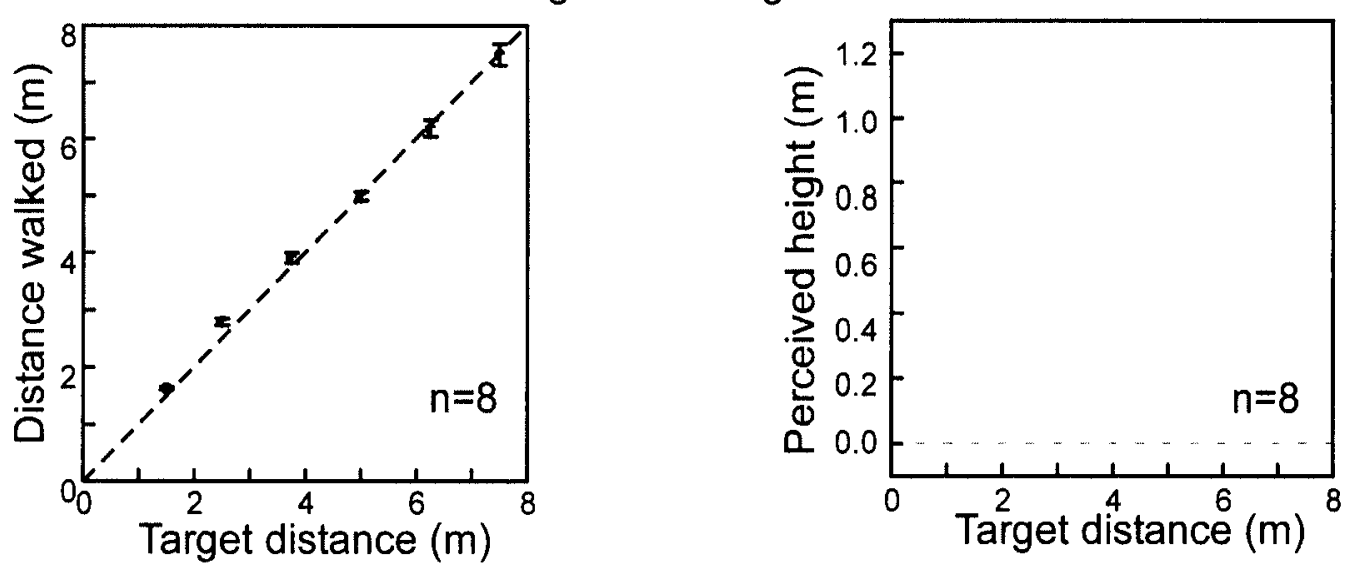

Target: $0.5 \mathrm{~m}$ above the ground
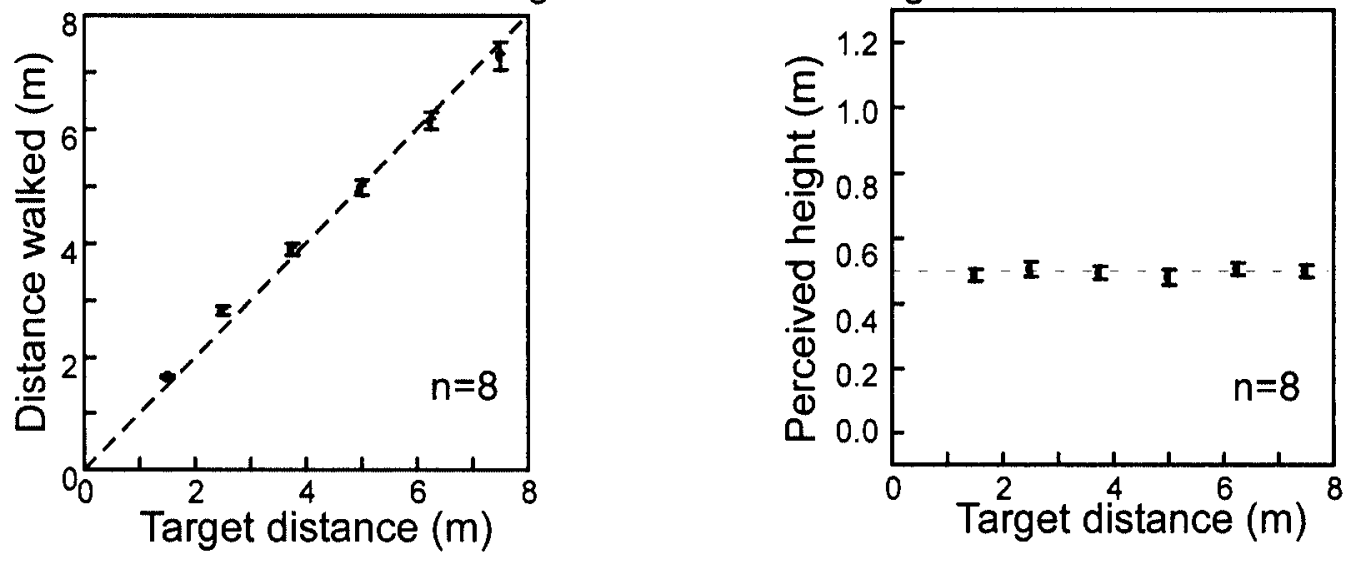

Target: $0.5 \mathrm{~m}$ below the eye level
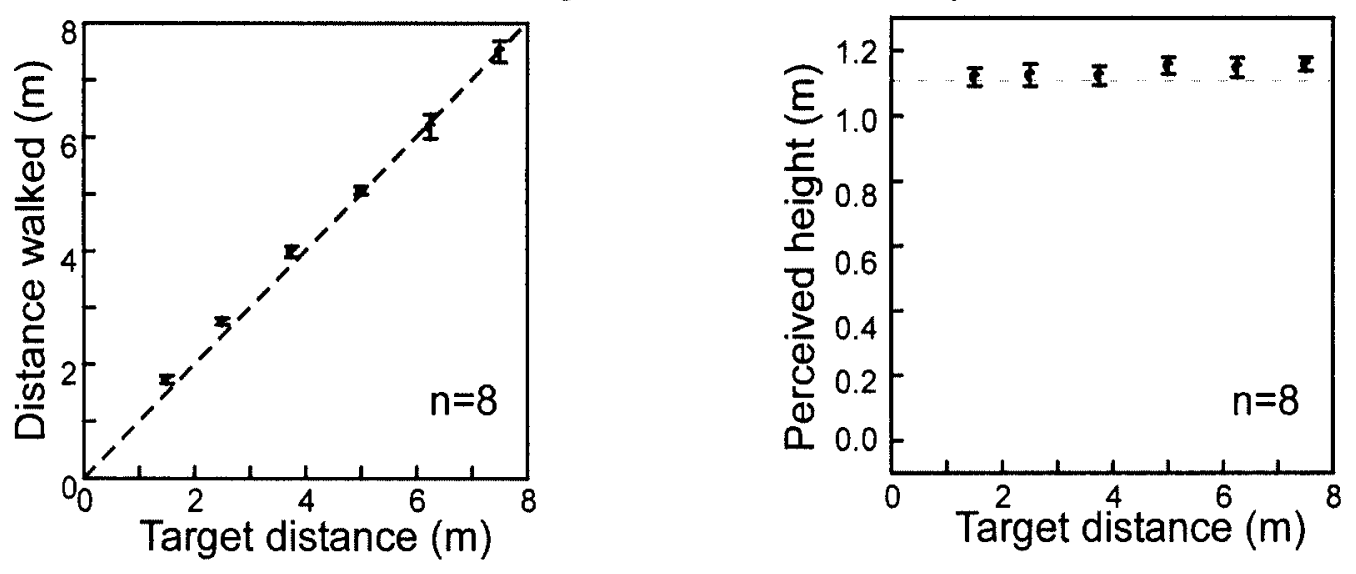

Figure 2.3. Distance and height judgments in the well-lit environment. Error bars represent $1 \mathrm{SE}$. 


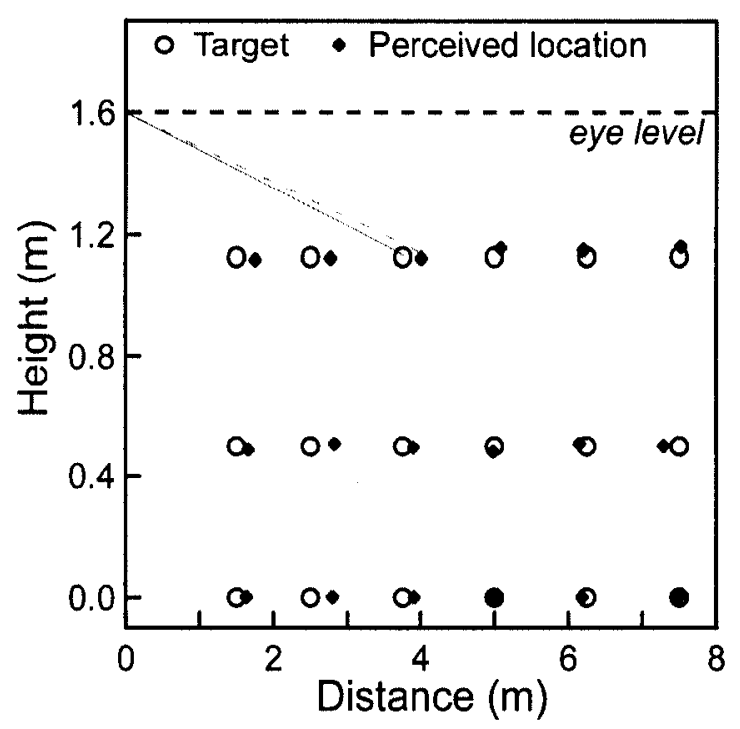

(a)

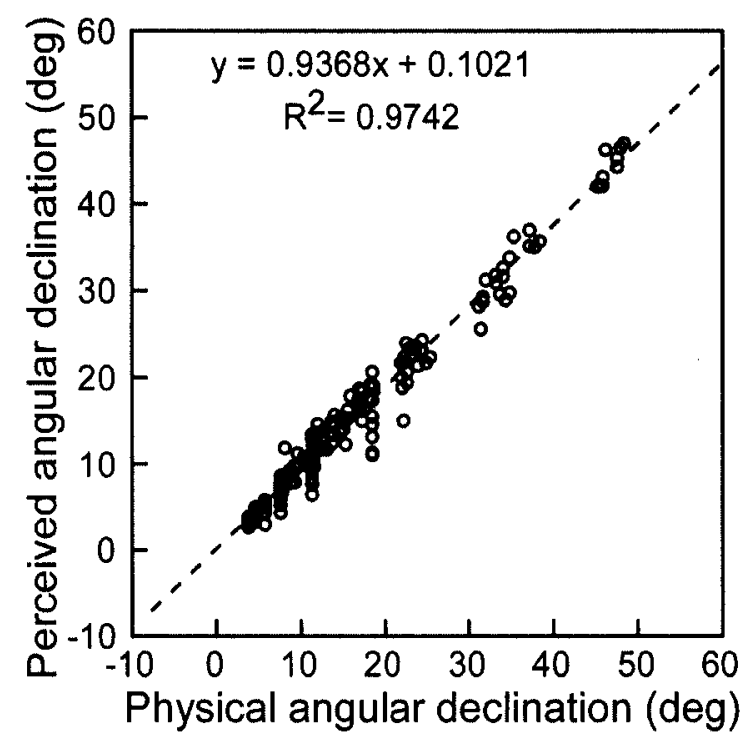

(b)

Figure 2.4. (a). The mean perceived location of the target at different height in the well-lit environment. (b). The perceived angular declination as a function of the physical angular declination. 
Target: On the ground
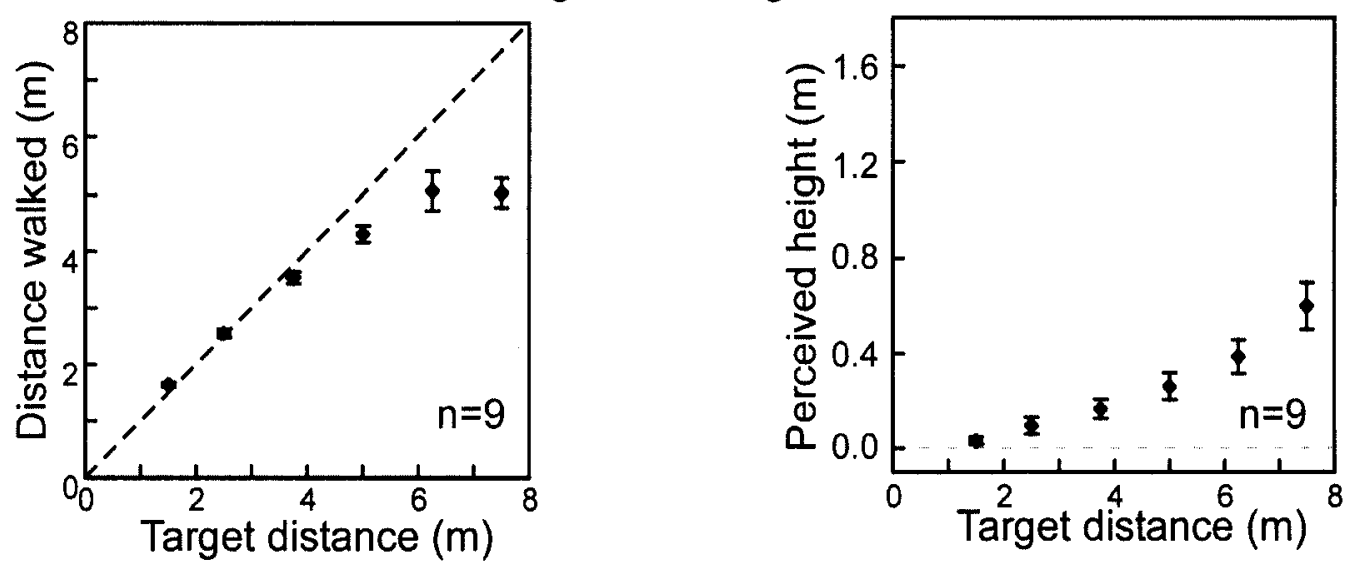

Target: $0.5 \mathrm{~m}$ above the ground
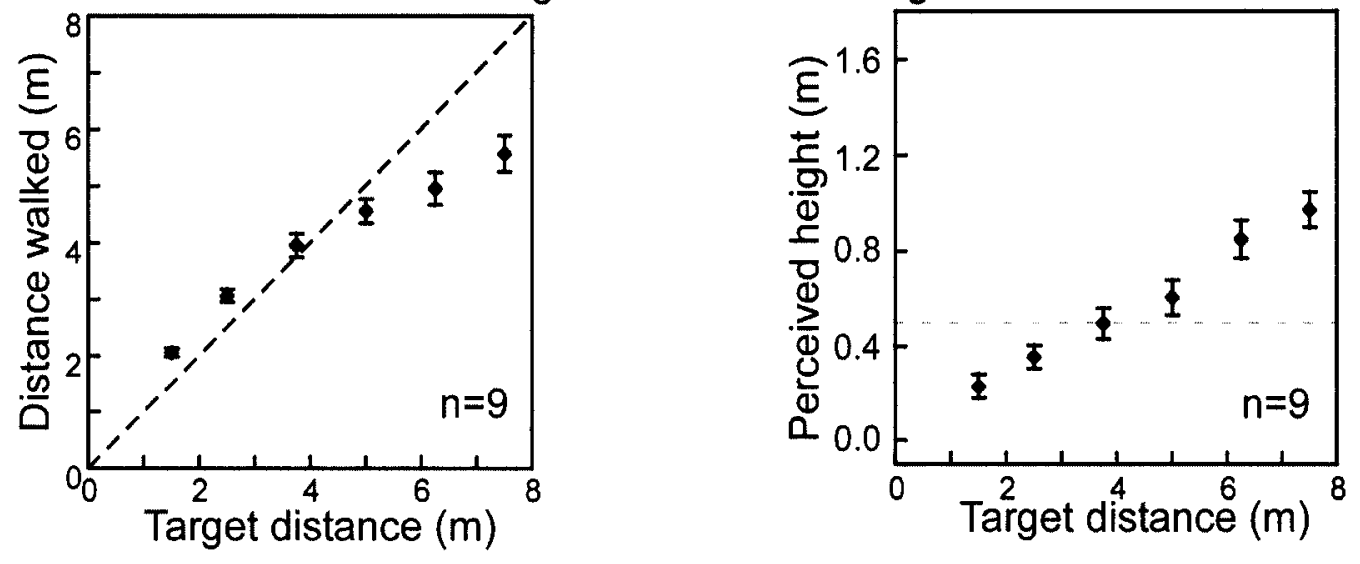

Target: $0.5 \mathrm{~m}$ below the eye level
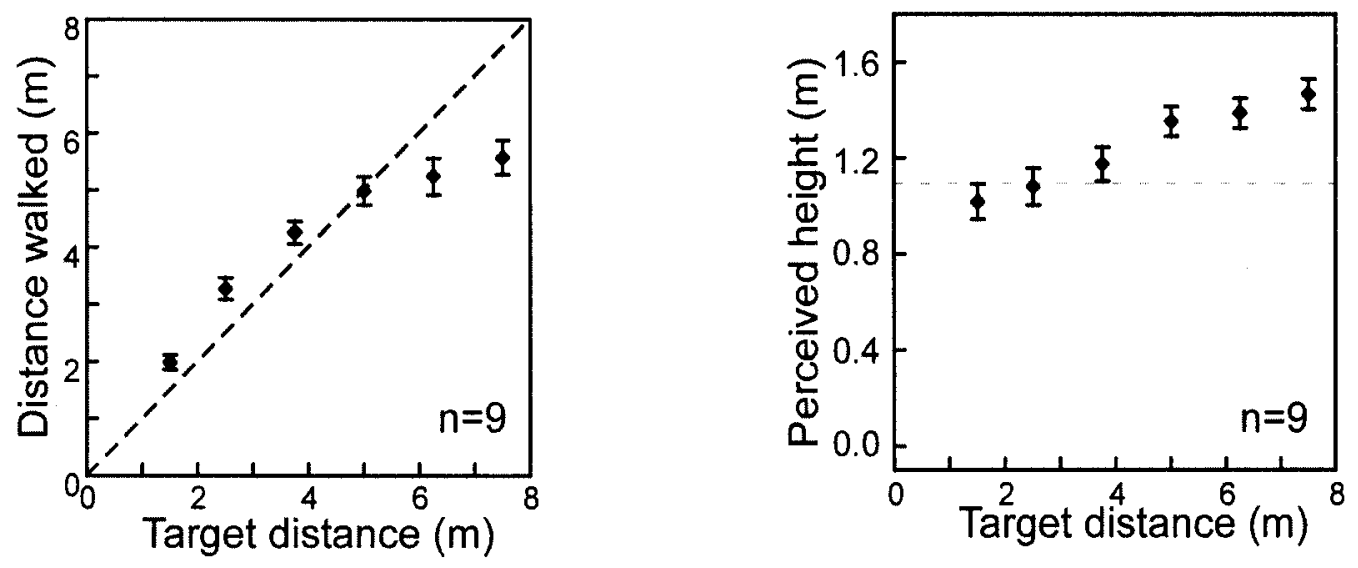

Figure 2.5. Distance and height judgments in the dark. Error bars represent $1 \mathrm{SE}$. 


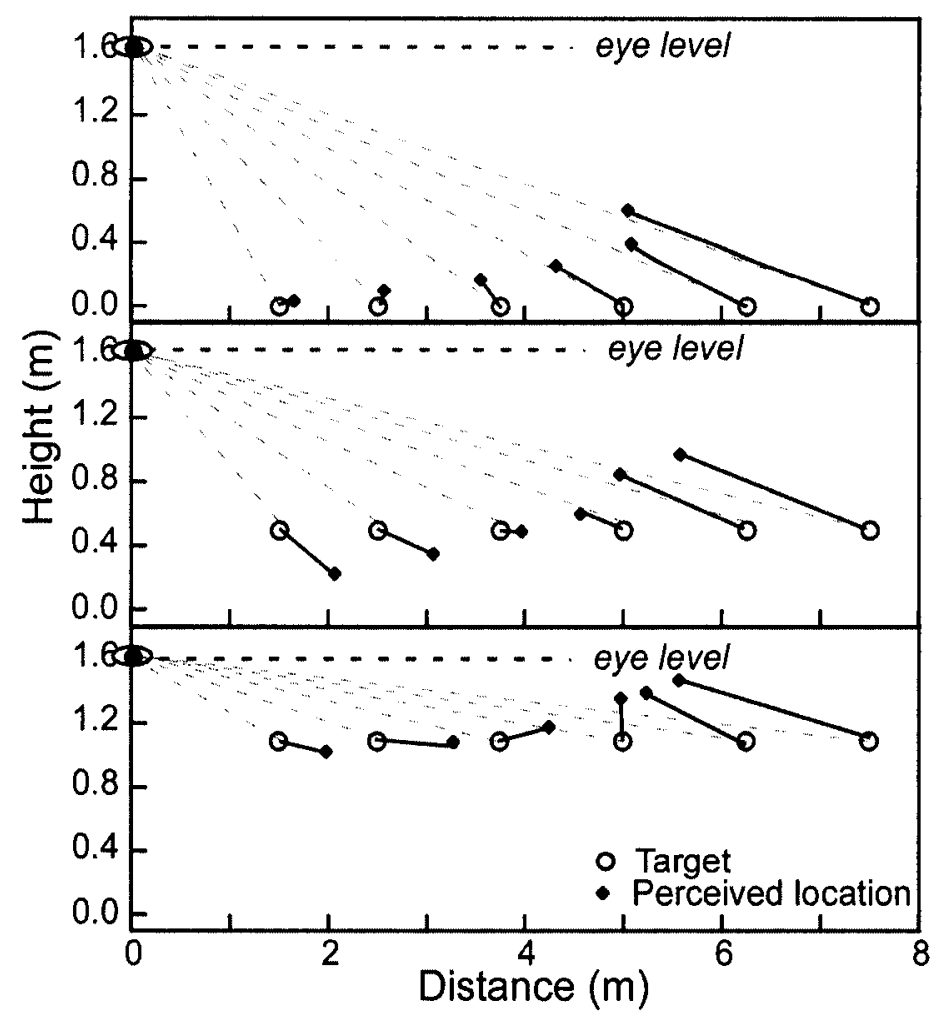

(a)

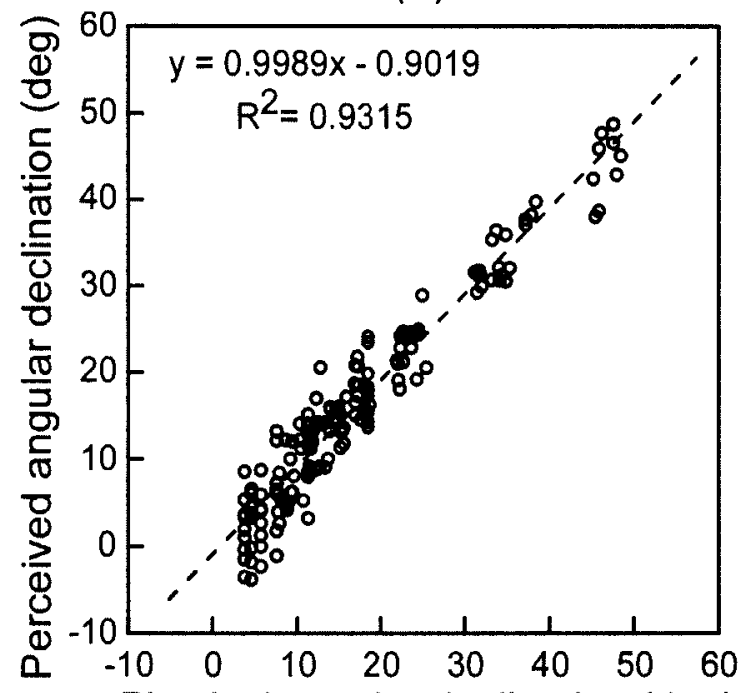

Physical angular declination (deg)

(b)

Figure 2.6. (a). The mean perceived location of the target at different height in the dark. (b). The perceived angular declination is plotted as a function of the physical angular declination. 

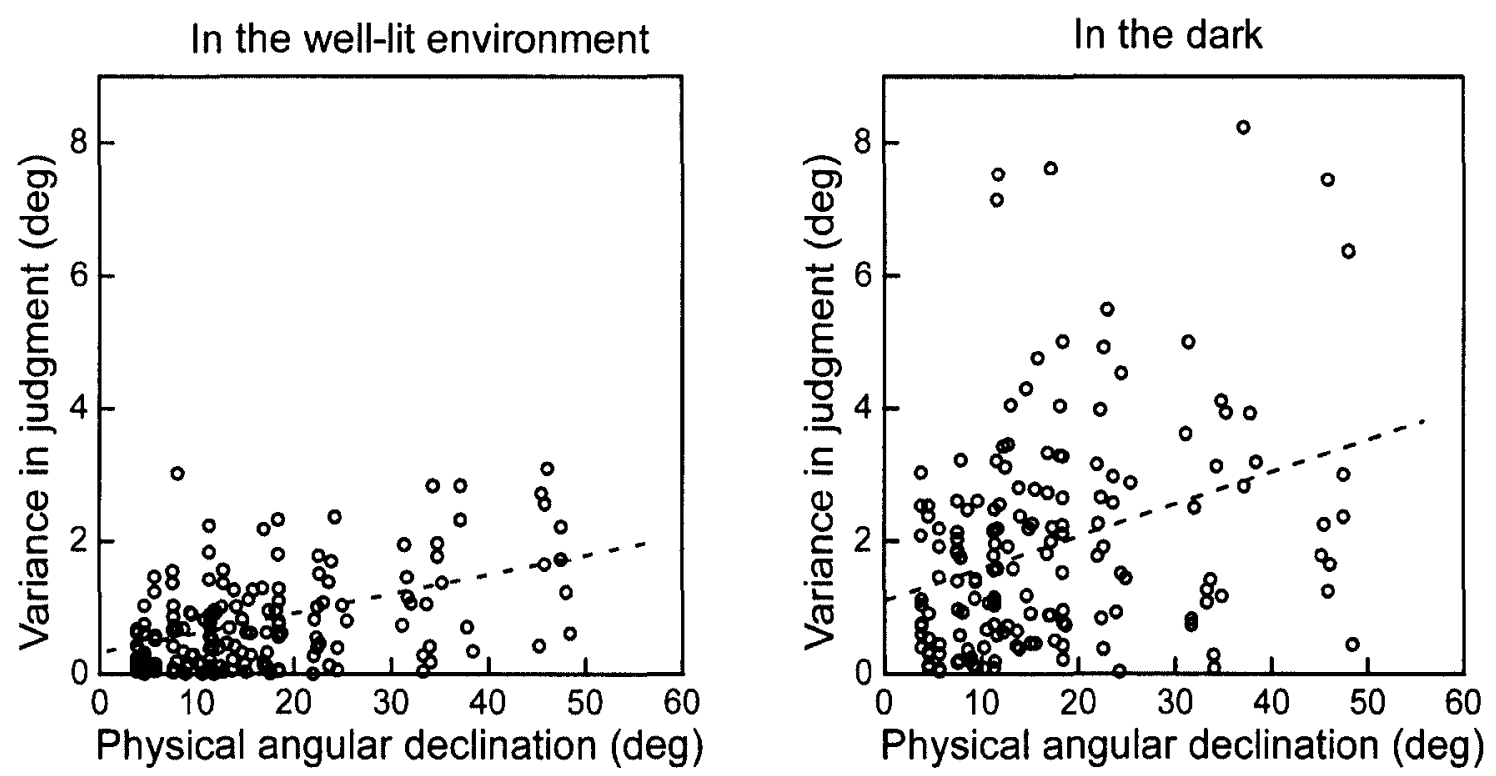

Figure 2.7. Variance in the angular declination perception as a function of the physical angular declination. 

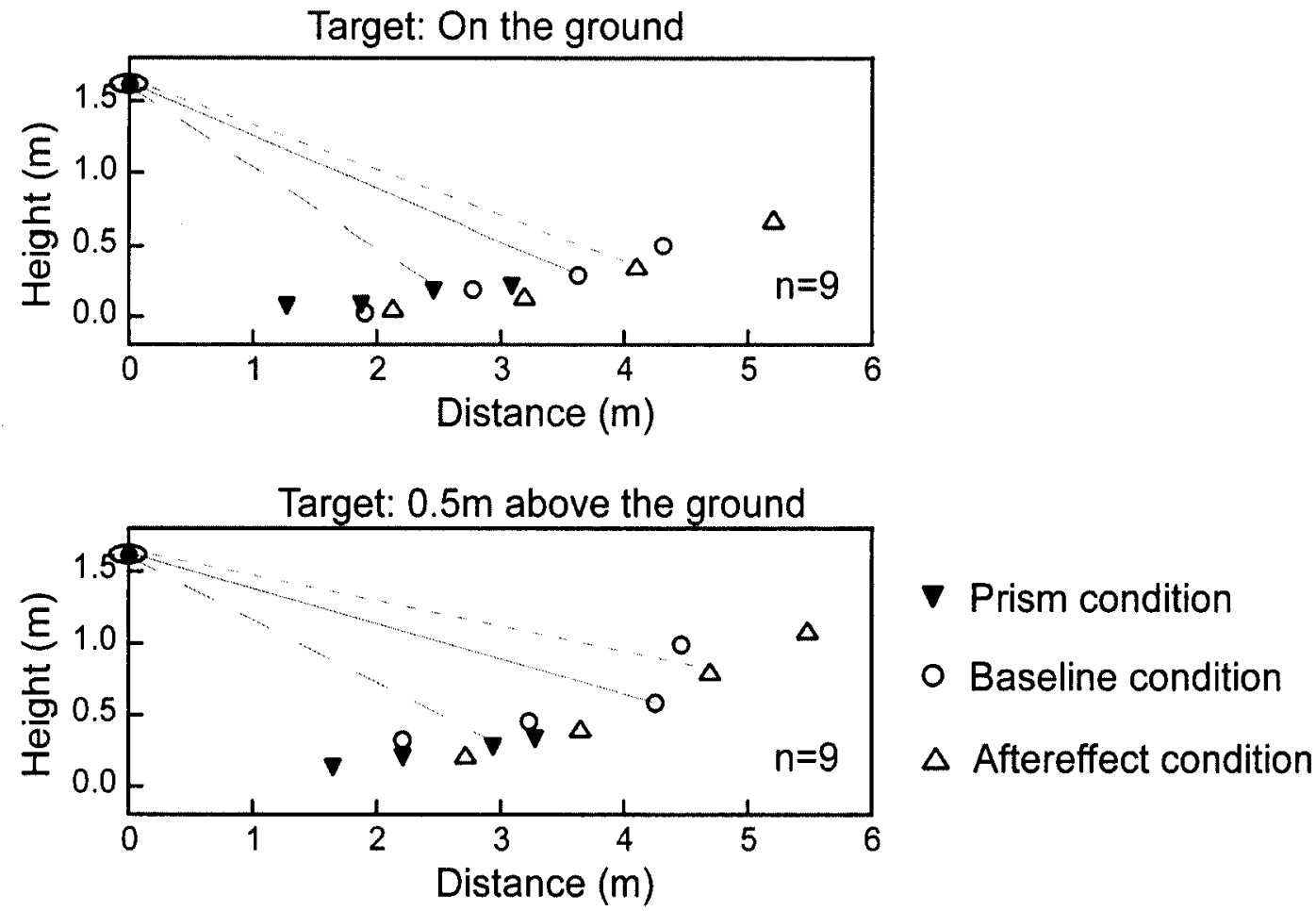

Target: $0.5 \mathrm{~m}$ below the eye level

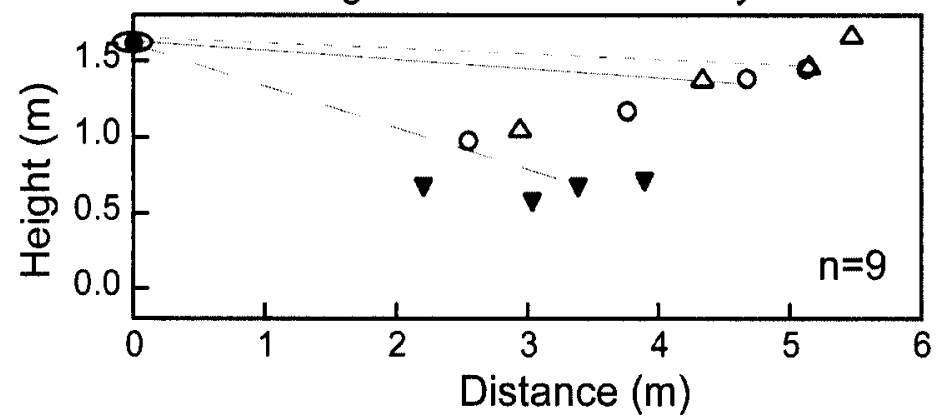

Figure 2.8. The mean perceived location of the target at different height in the baseline, prism, and aftereffect conditions. 


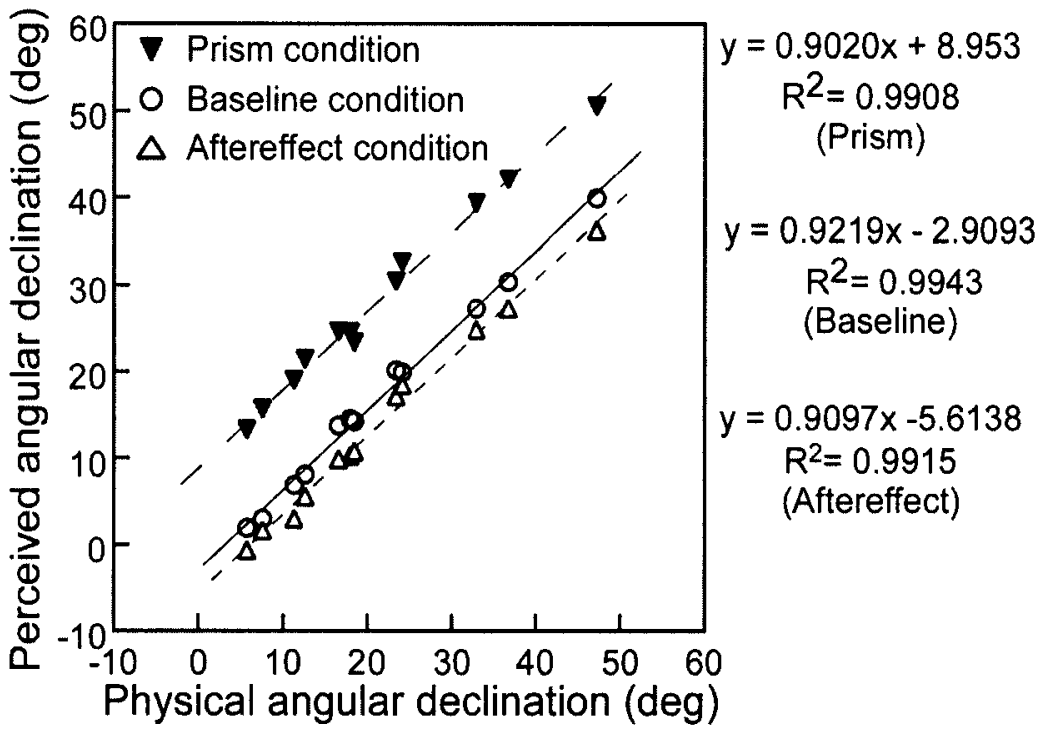

(a)

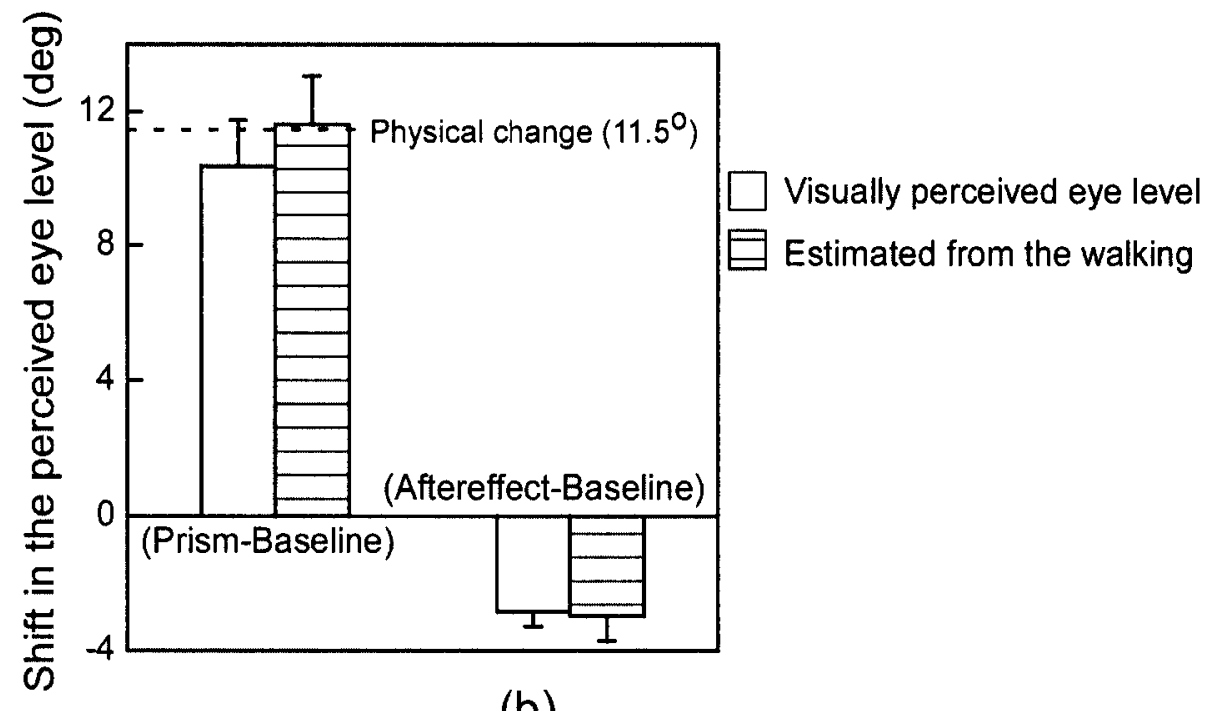

(b)

Figure 2.9. (a). The mean perceived angular declination as a function of the physical angular declination in the baseline, prism, and aftereffect conditions. (b). Shifts in the perceived eye level in the prism and aftereffect conditions as compared to the baseline condition. 


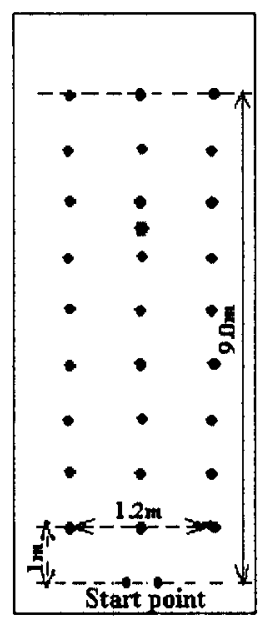

(a)

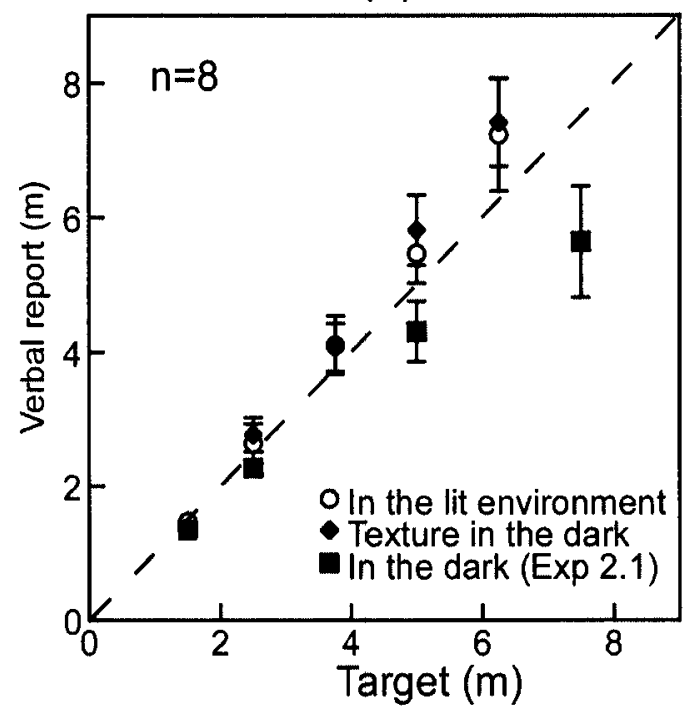

(b)

Figure 3.1. (a). The experimental settings in the dark. (b). When the ground in the dark was uniformly textured with a grid pattern, subjects' judgment of distance was comparable with that in the lit environment. Error bars represent $1 \mathrm{SE}$. 

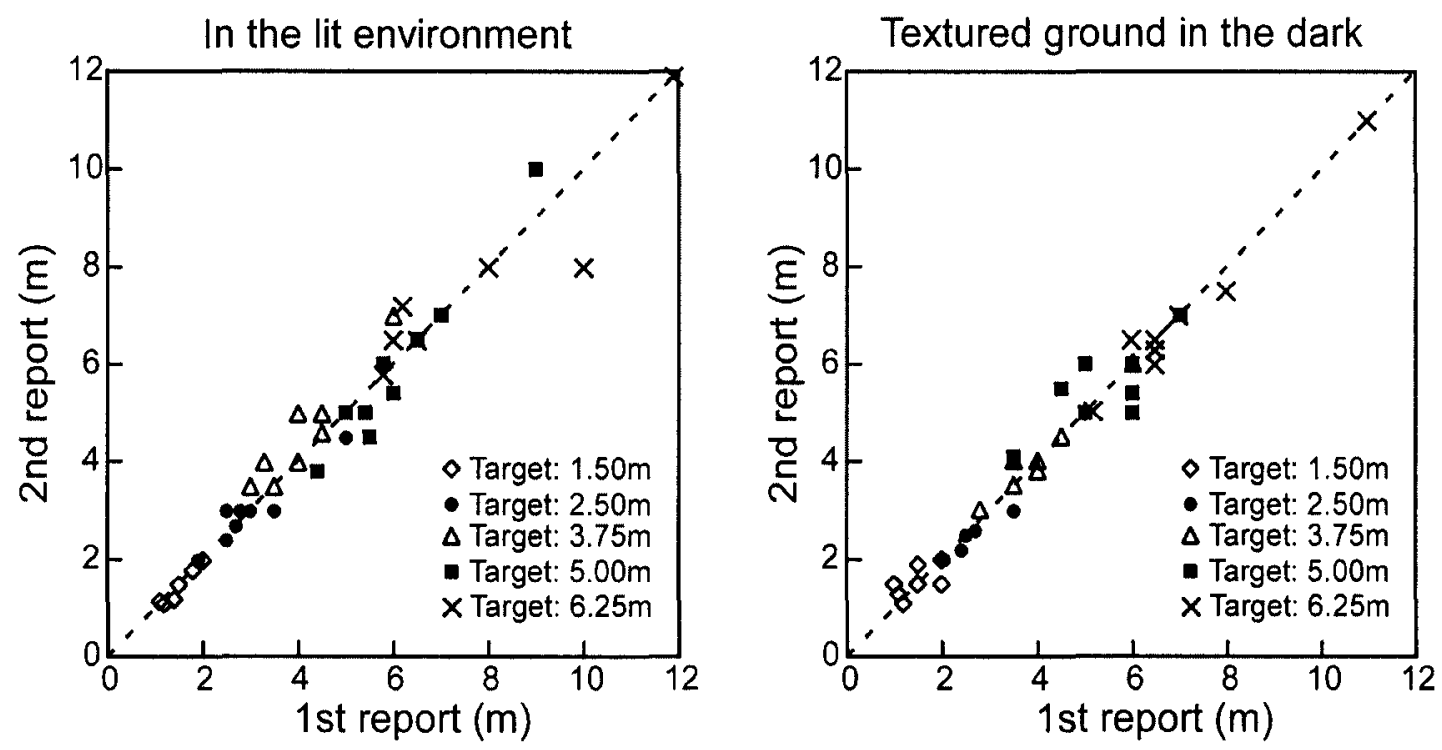

Figure 3.2. Pairs of estimates of the target distance are plotted against each other to illustrate the Test-Retest reliability of the measurement. 

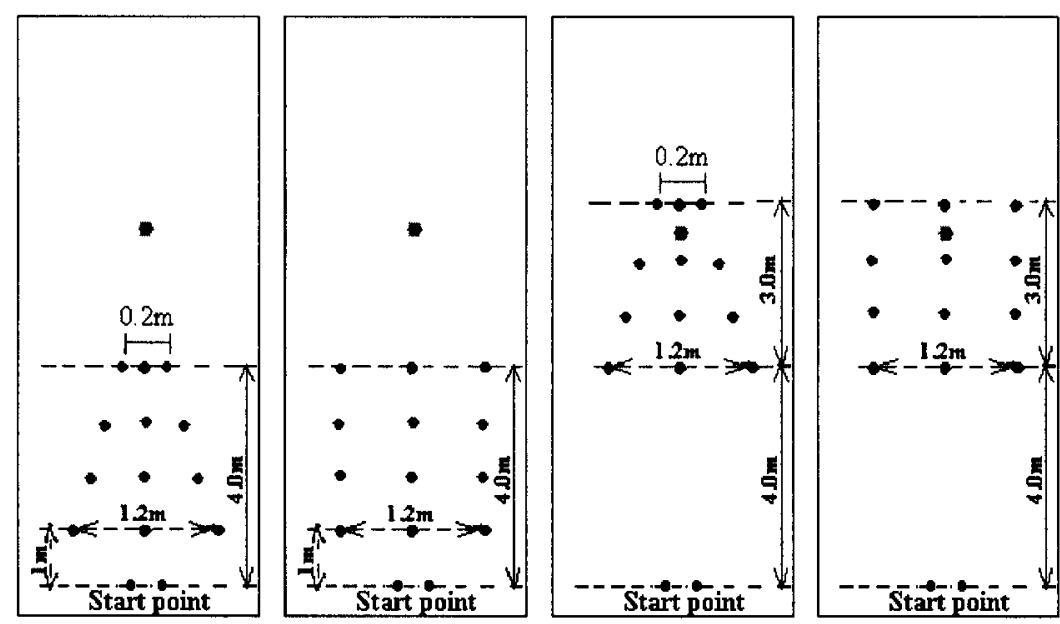

(a)

Texture location: Near
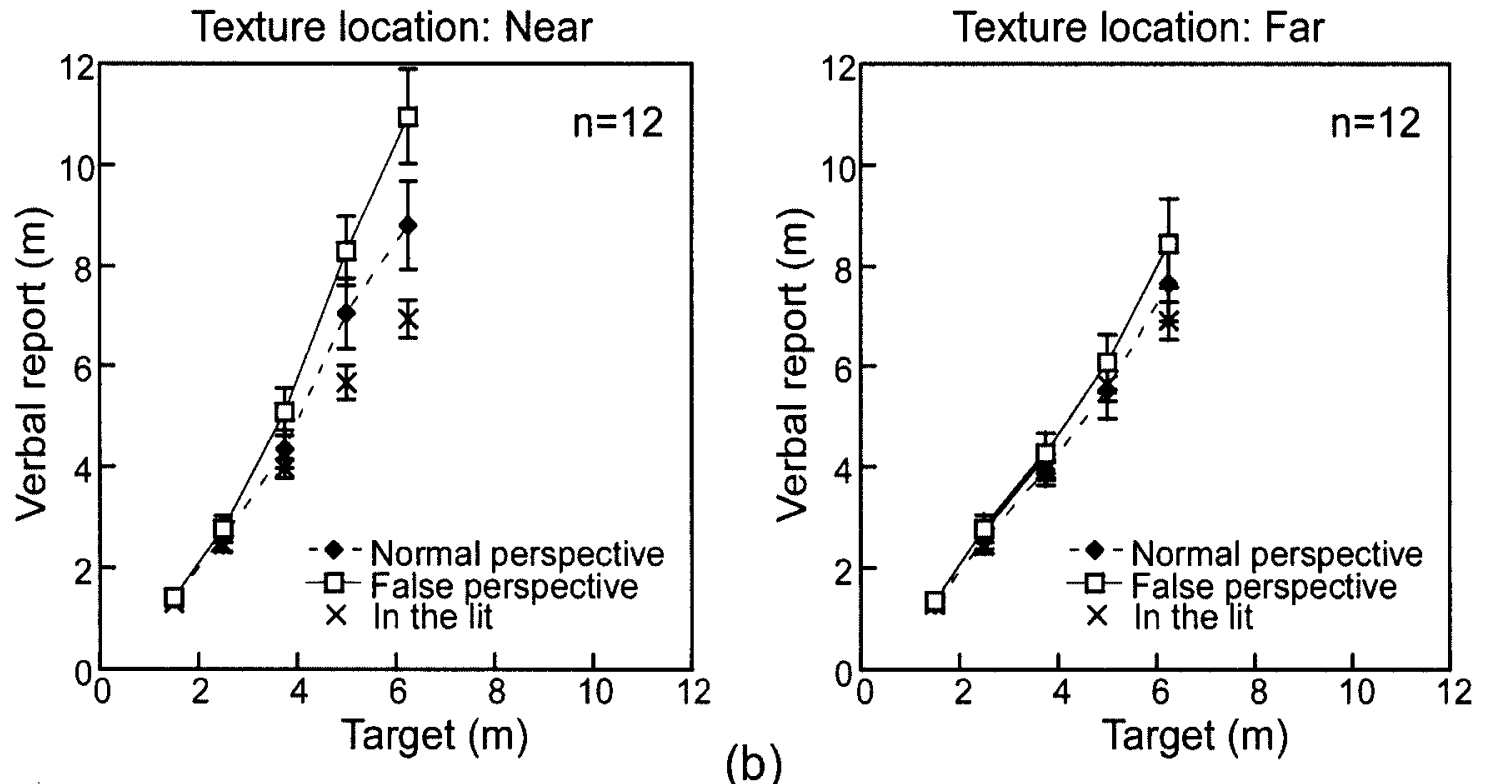

(b)

Figure 3.3. (a). The experimental settings. (b). Distance judgment in the presence of normal or false linear perspective. Error bars represent 1 SE. 

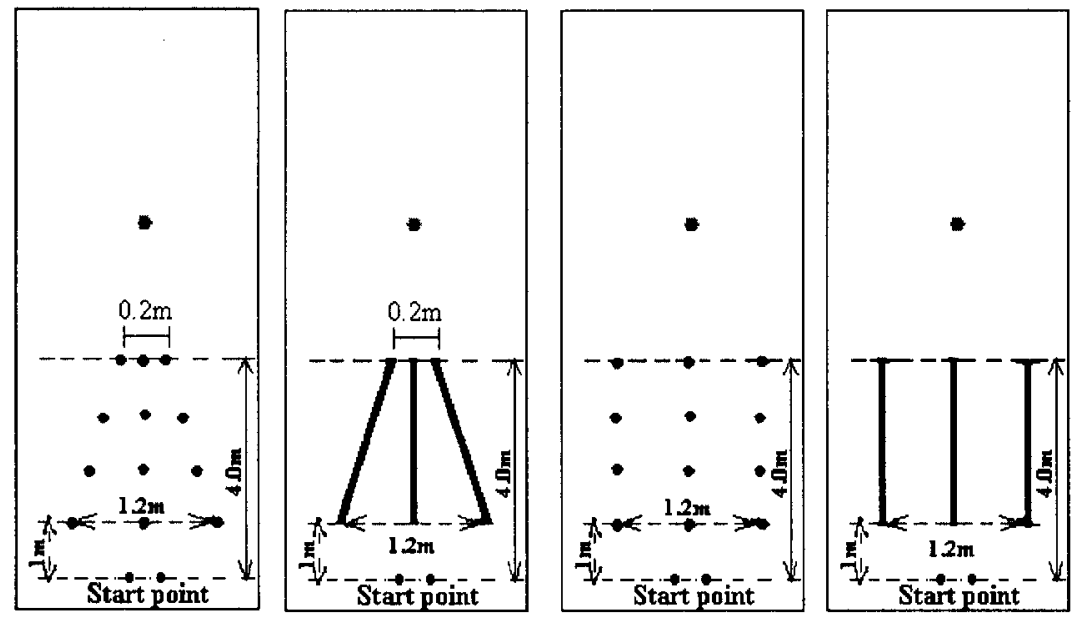

(a)

False perspective

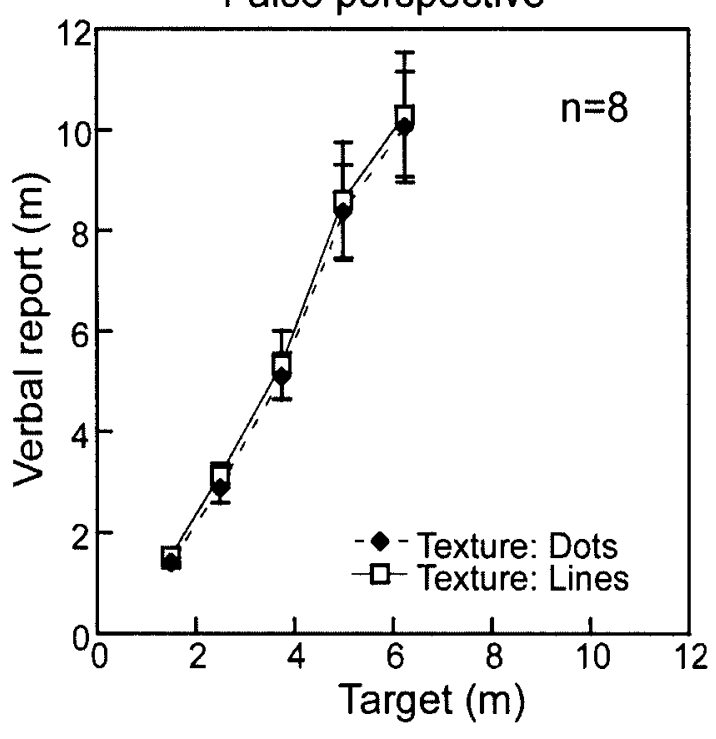

(b)

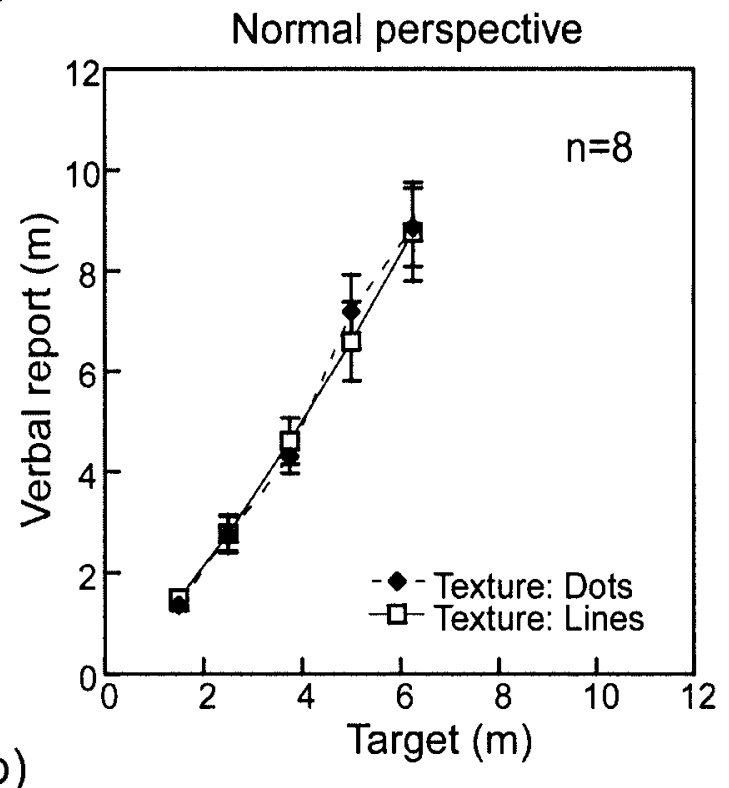

Figure 3.4. (a). The experimental settings. (b). Subject's distance estimates were nearly identical with or without the presence of valid texture compression cue. Error bars represent $1 \mathrm{SE}$. 

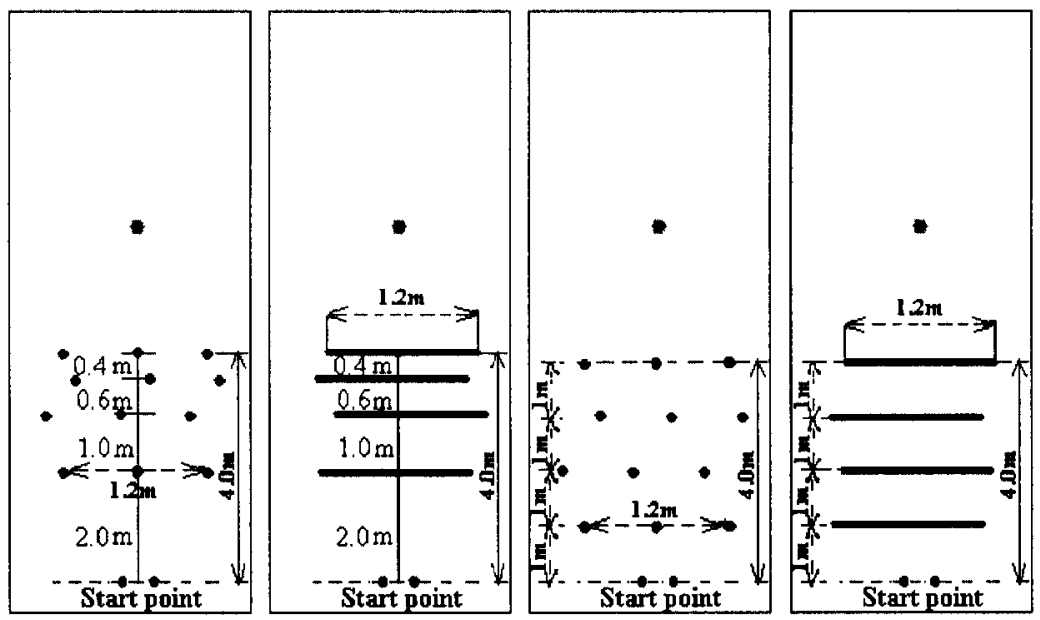

(a)
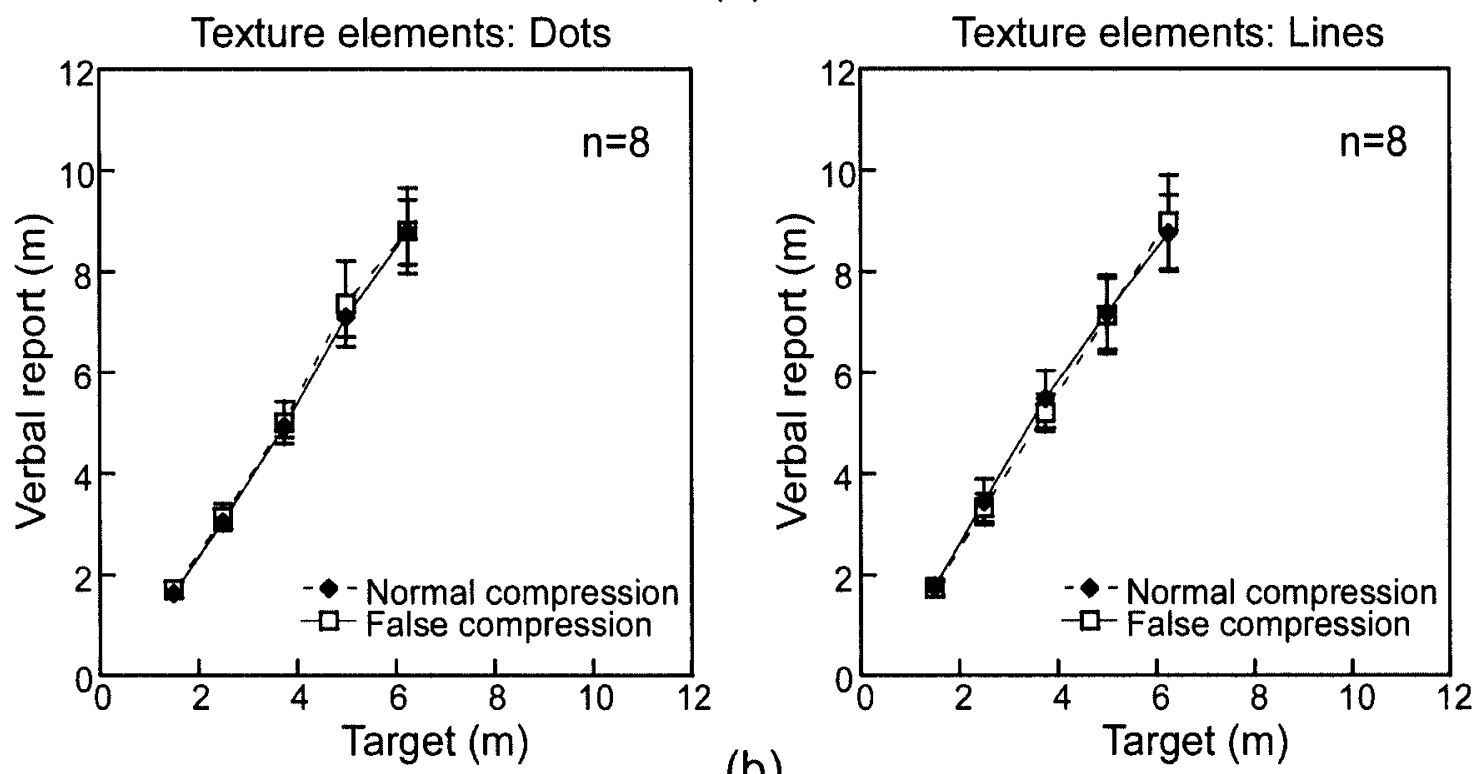

(b)

Figure 3.5. (a). The experimental settings. (b). False texture compression had little influence on subjects' distance perception. Error bars represent $1 \mathrm{SE}$. 

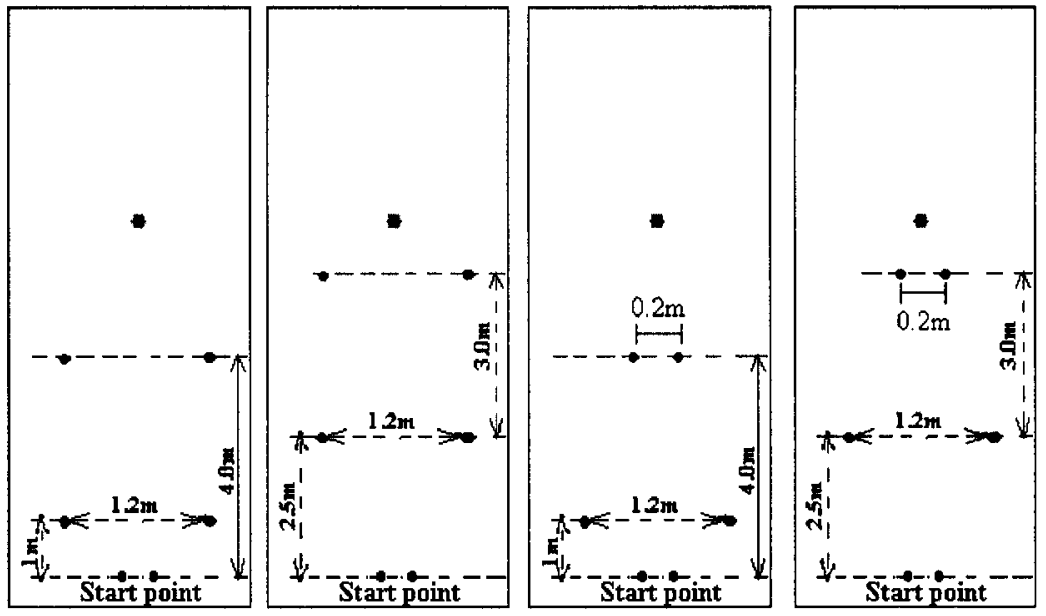

(a)
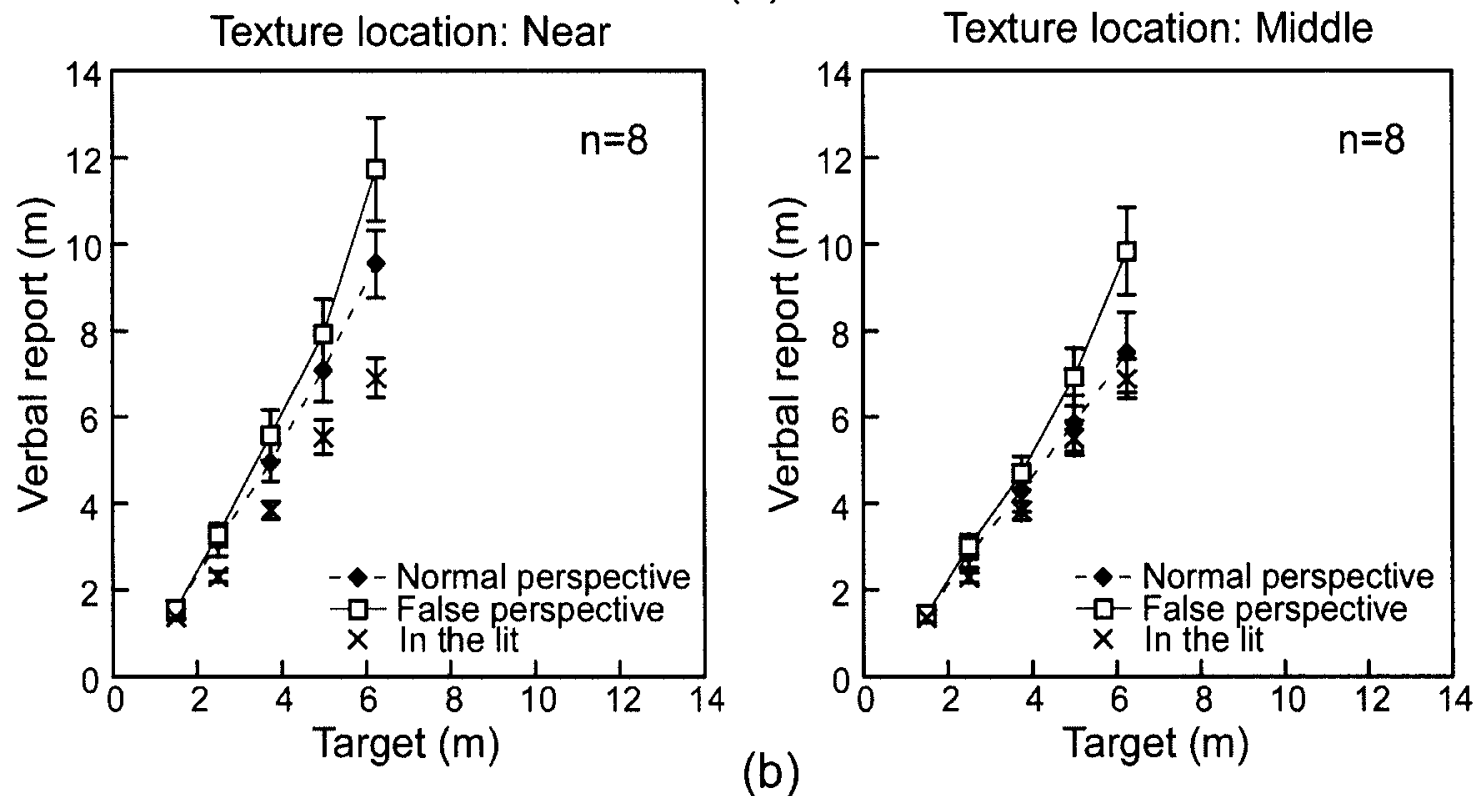

Figure 3.6. (a). The experimental settings. (b). Significant distance overestimation was obtained from minimal false linear perspective information. Error bars represent $1 \mathrm{SE}$. 

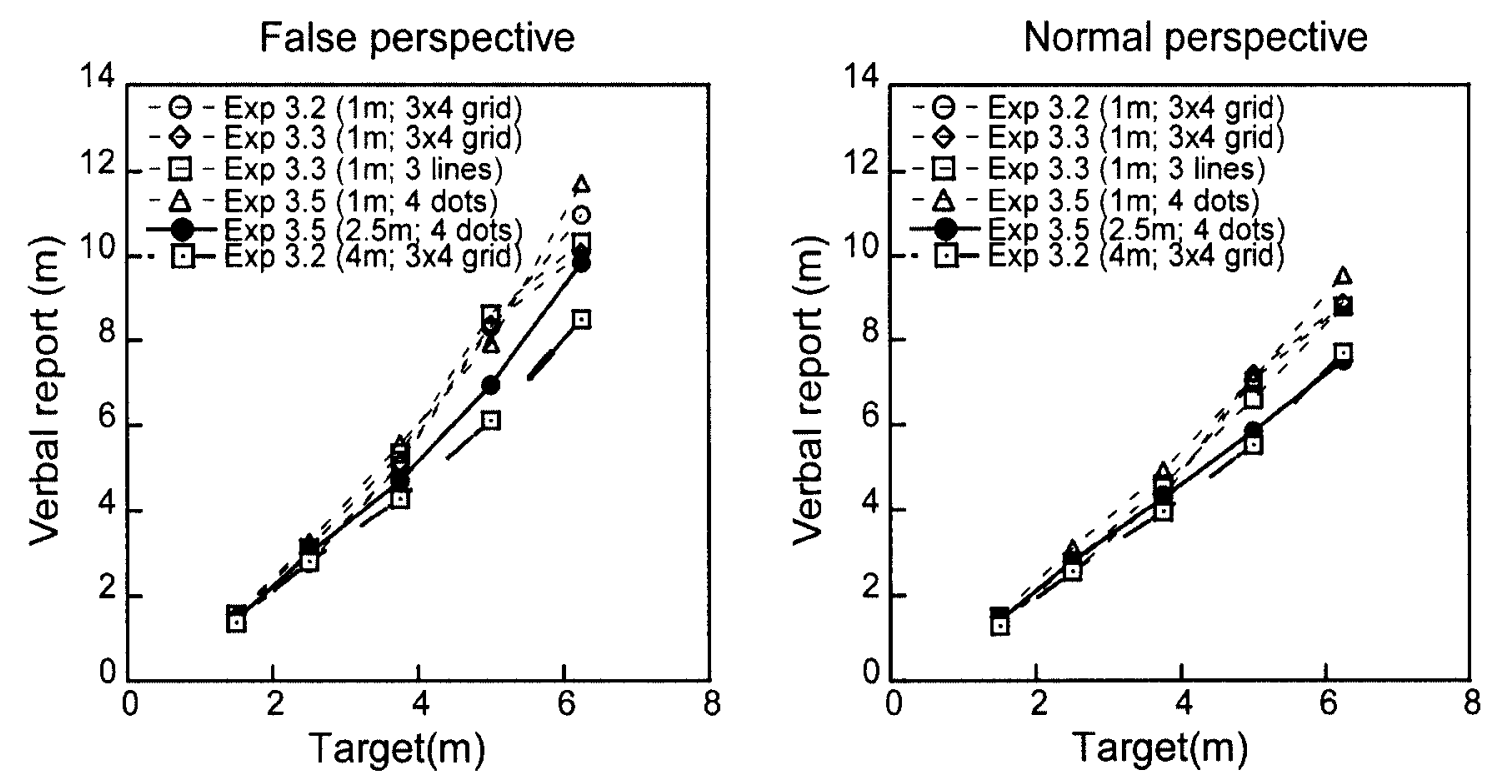

Figure 3.7. Data from previous experiments were plotted together to show the location effect of texture pattern on distance perception. 

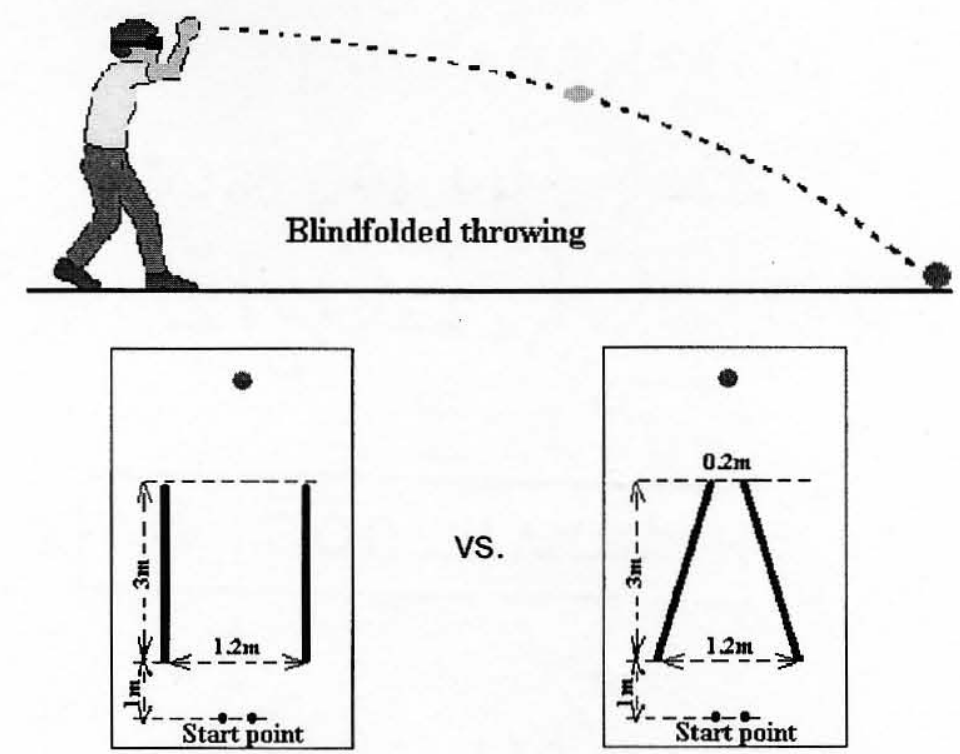

(a)

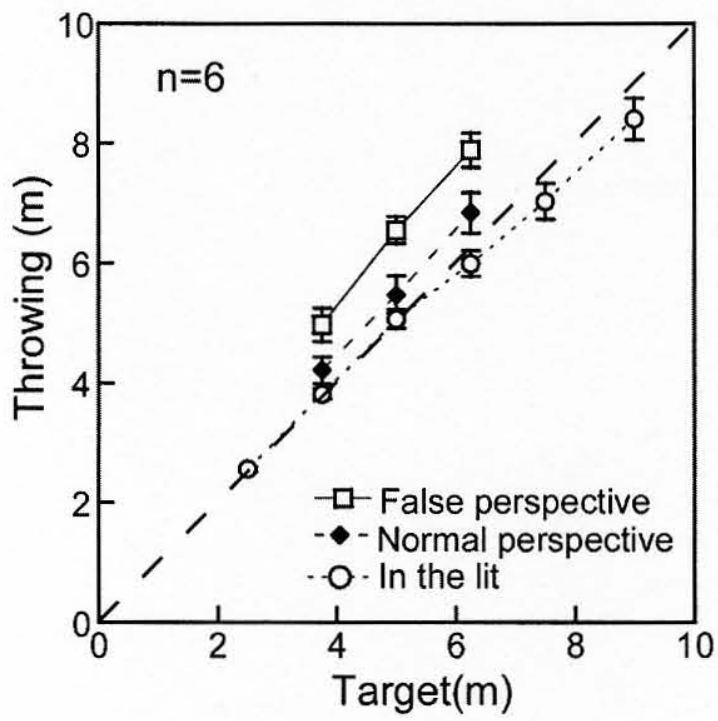

(b)

Figure 3.8. (a). The blindfolded throwing paradigm and the experimental settings.

(b). Significant distance overestimation caused by false linear perspective was also obtained in the blindfolded throwing task. Error bars represent $1 \mathrm{SE}$. 


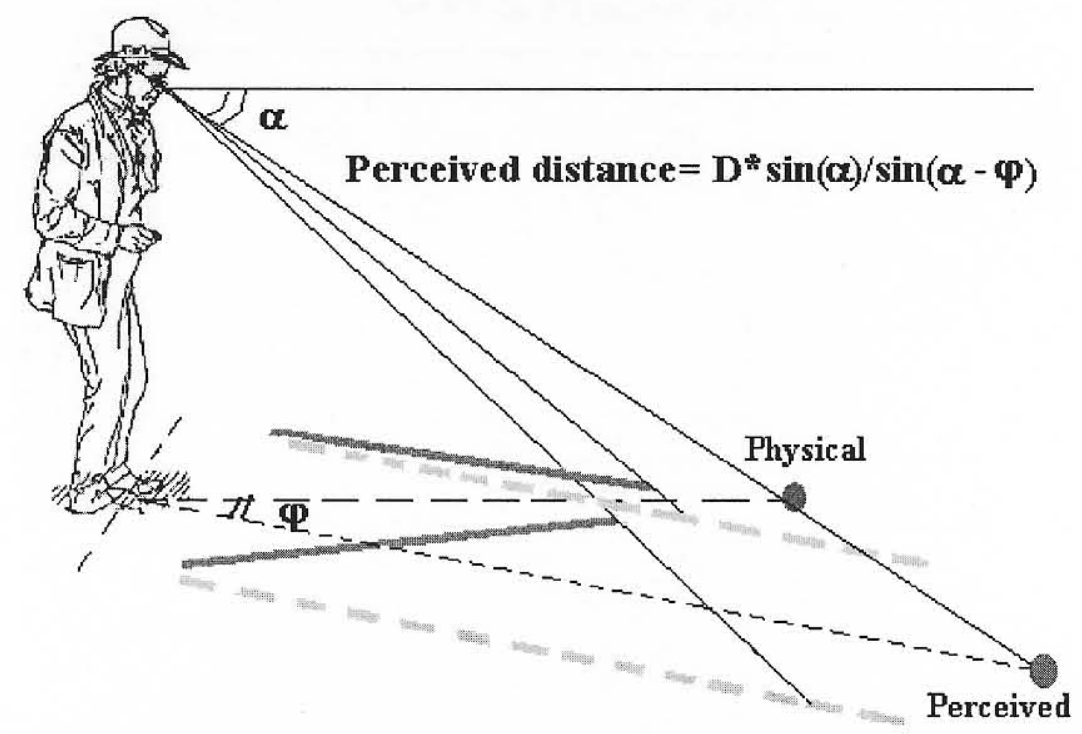

Figure 3.9. Distance overestimation caused by false linear perspective may be explained by the misrepresentation of the ground surface. 


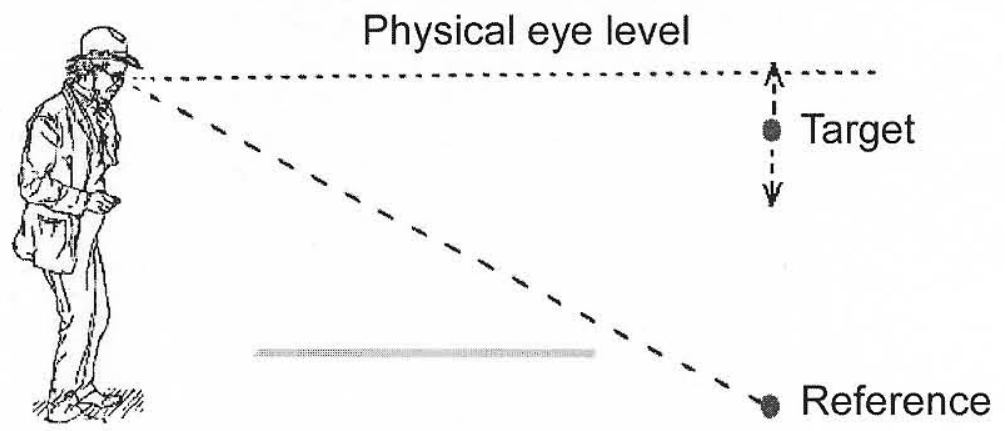

(a)
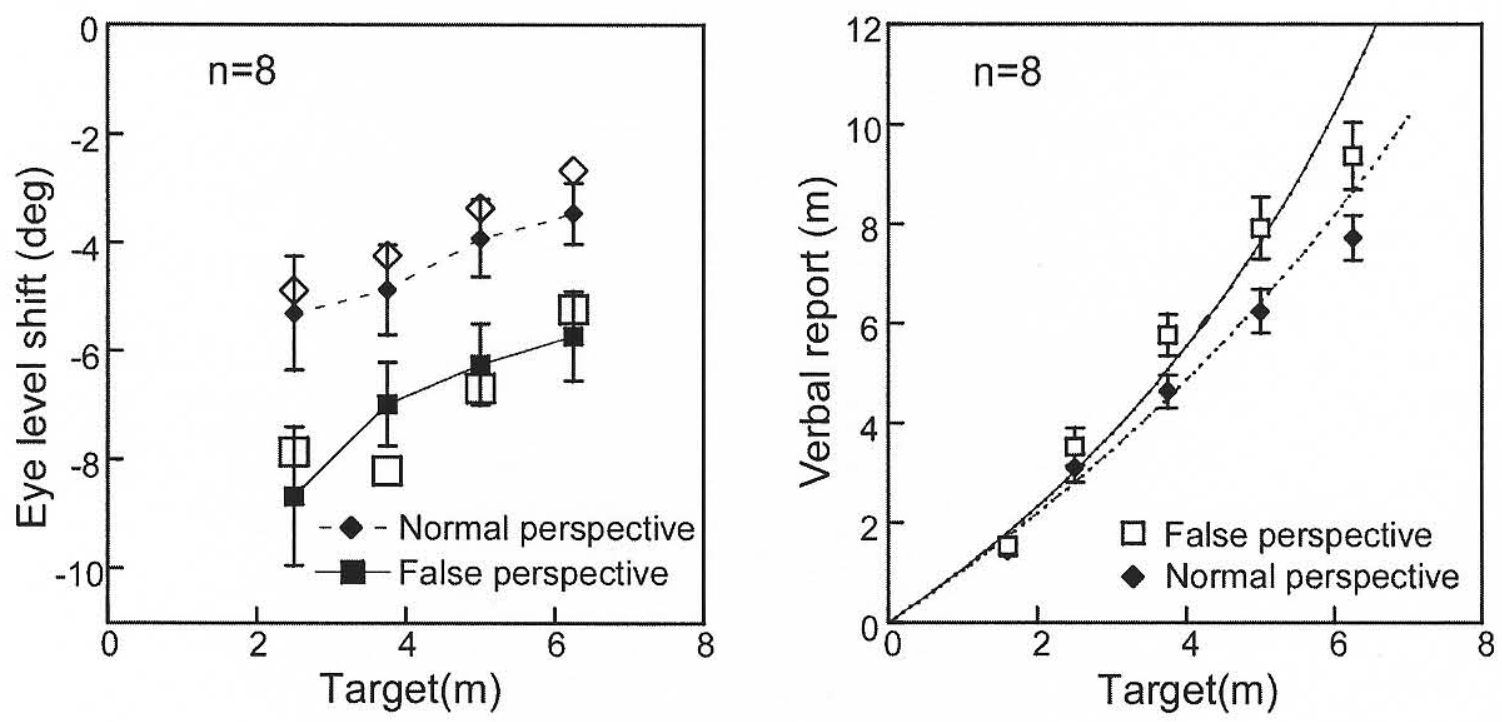

(b)

Figure 3.10 (a). The experimental settings for measuring the subjects' perceived eye level. (b). Left: Subjects' perceived eye level. The open symbols were the perceived slant estimated from distance perception. Right: Subjects' judgments of distance. The trend lines were fitted using the eye shift data (filled squares and diamonds) in the left figure. Error bars represent 1 SE. 


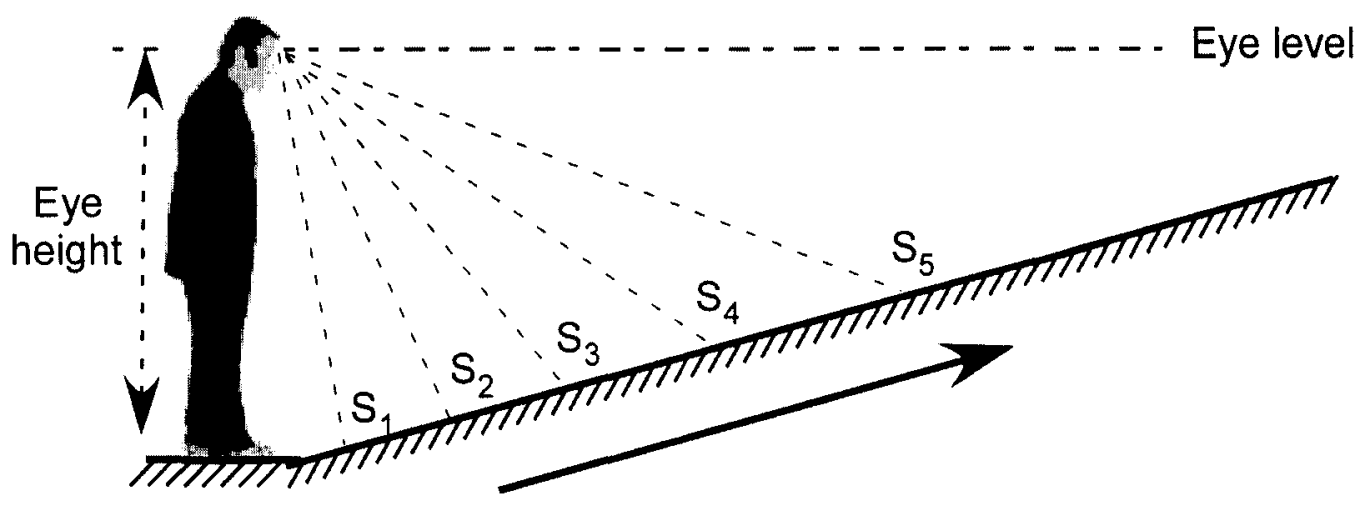

Figure 4.1 The Sequential Surface Integration Process hypothesis. The SSIP initiates the computation from the near space where rich depth cues make veridical perception possible, gradually extends it to distant areas, and eventually integrates all local areas into an overall surface representation. 


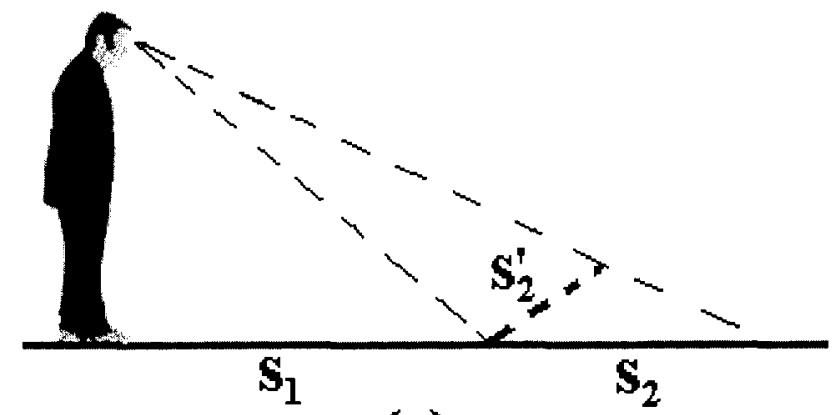

(a)

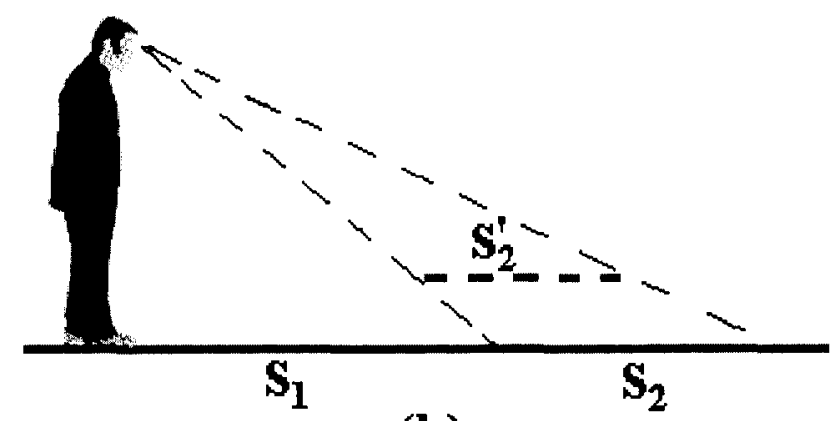

(b)

Figure 4.2 Always using the accurately perceived near surface area $\left(S_{1}\right)$ as the reference for further computation of distant areas $\left(S_{2}\right)$, the SSIP is able to correct the "frontal shift" in slant perception (a) and disambiguate location uncertainty (b). 


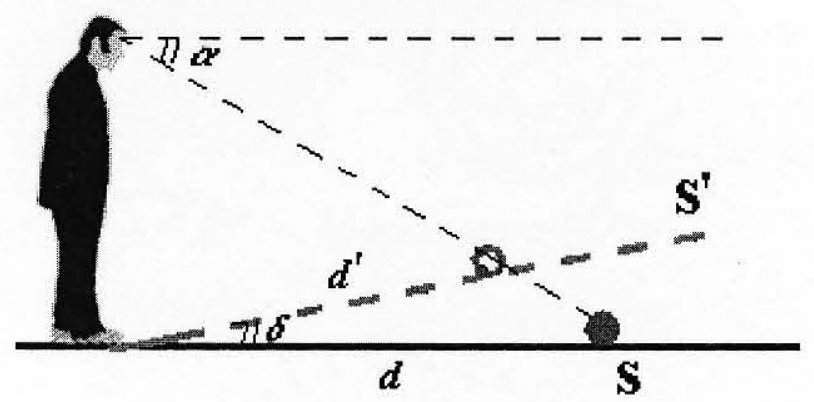

(a)

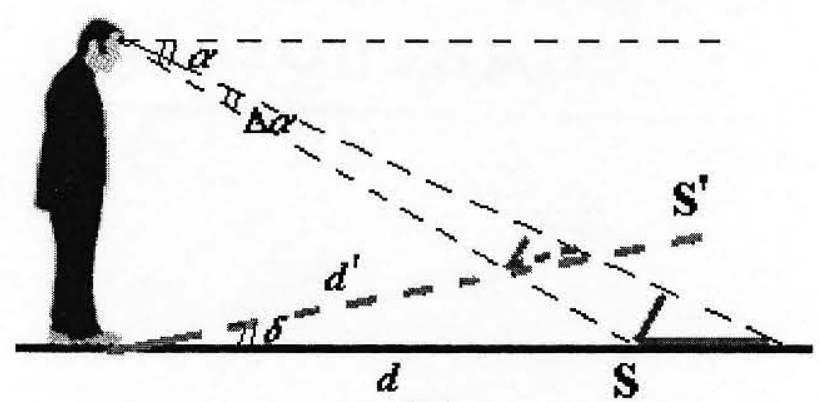

(b)

Figure 4.3. Without the accessibility of near depth cues, the perception of the ground surface will be influenced by the "frontal shift" tendency in surface perception. Egocentric distance is thus underestimated (a). Relative distance judgment (the perceived aspect ratio of the L-shape) is also influenced (b). 


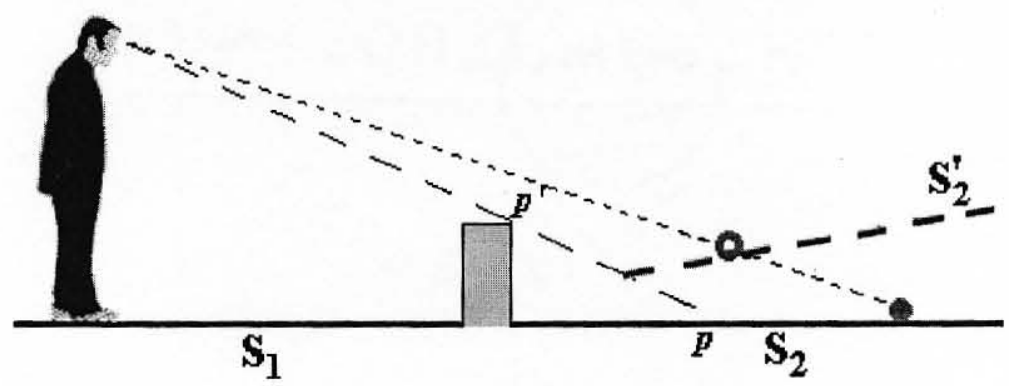

(a)

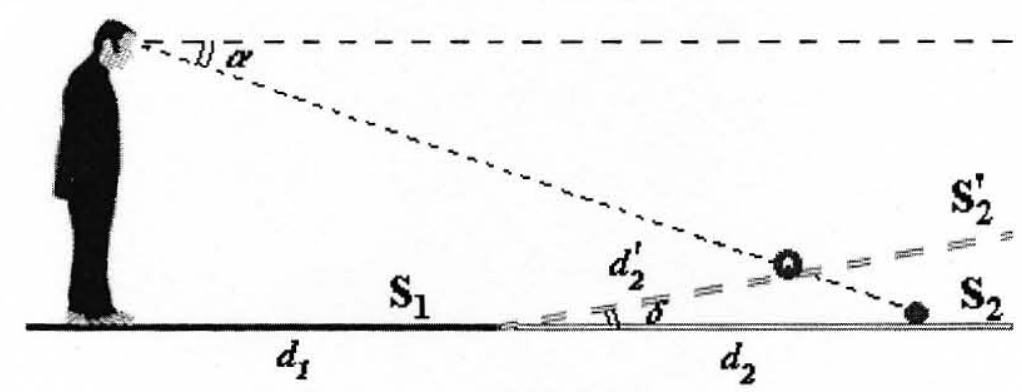

(b)

Figure 4.4. In the case that the surface continuity is disrupted, the integrative precess stops at the boundary and the perception of the surface beyond the disruption relies solely on the information on it. The distance and surface slant is thus misperceived. 


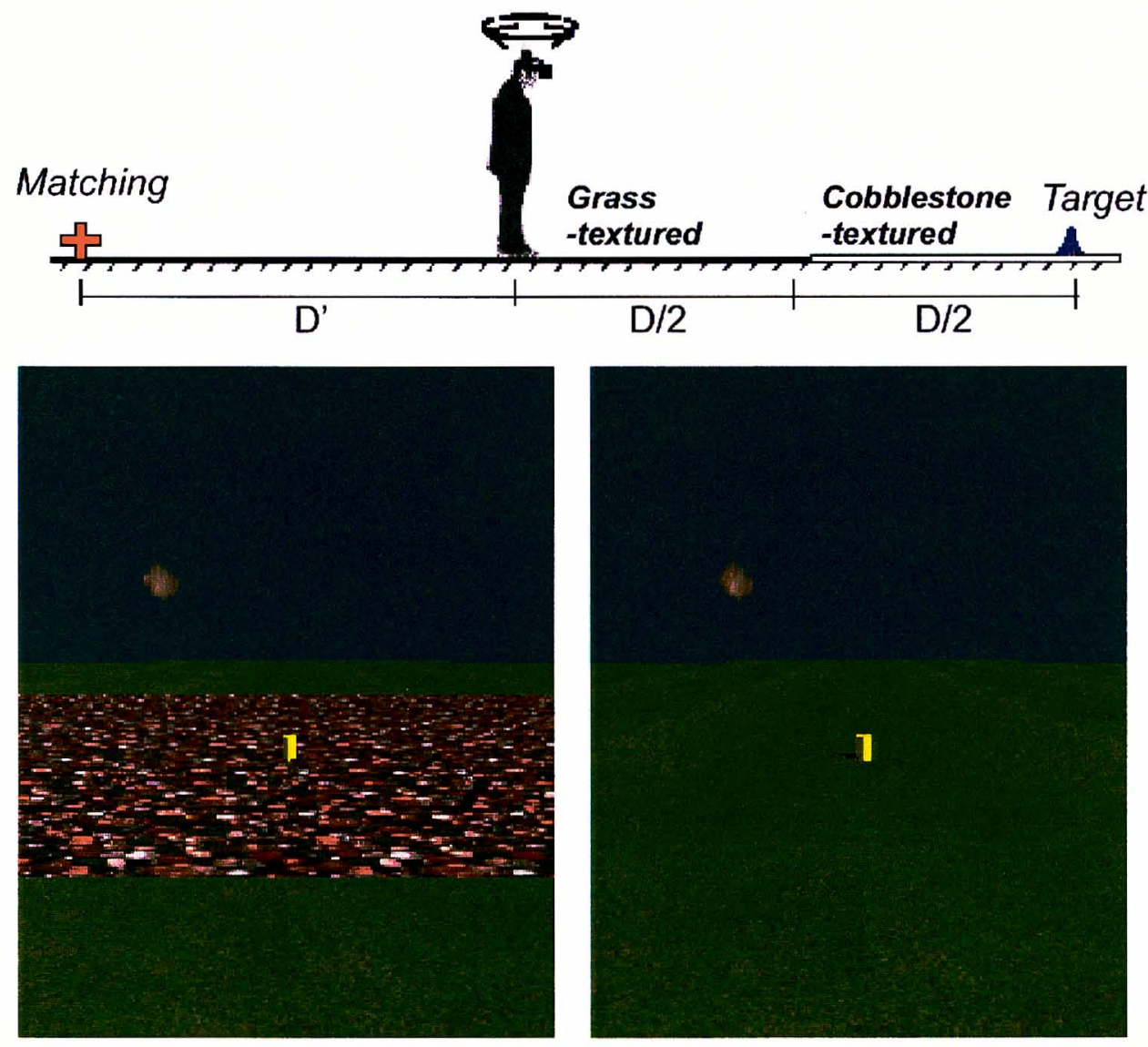

Figure 5.1. The settings and screen snapshots of the texture-discontinuity experiment. 

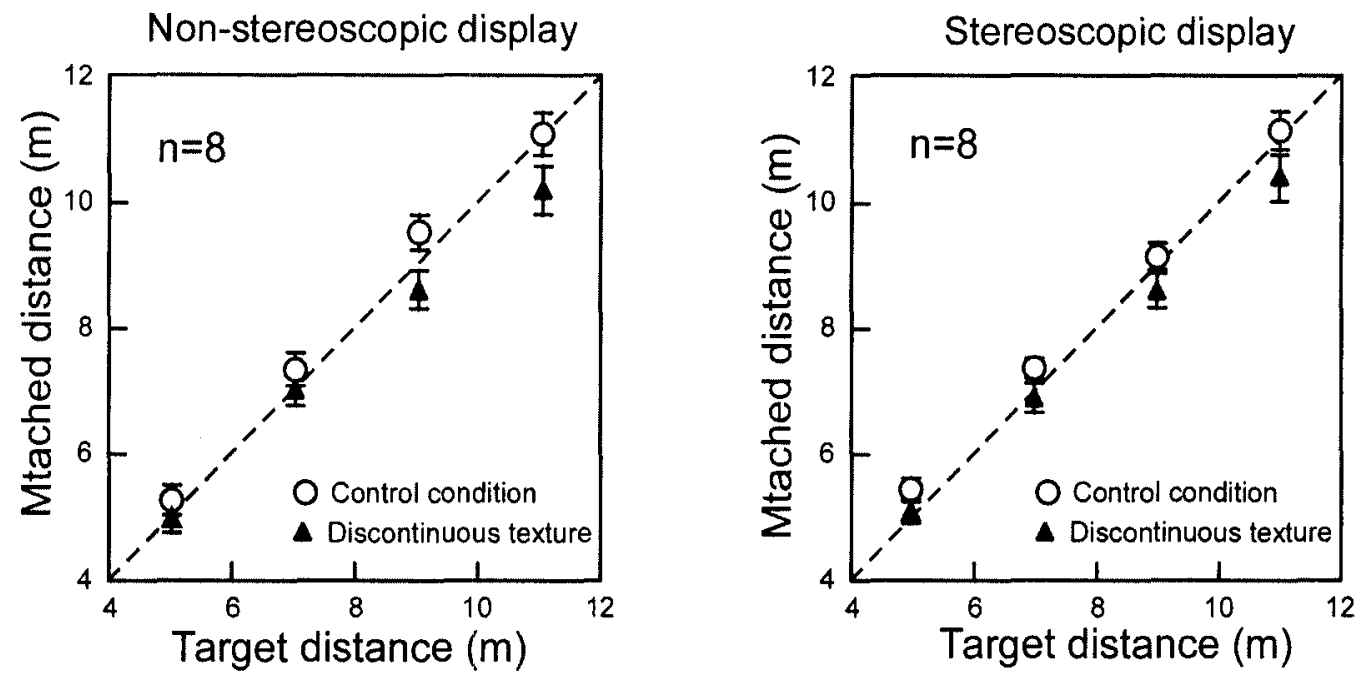

Figure 5.2. The texture-discontinuity effect on distance perception. Subjects' estimates of distance was measured using the perceptual matching paradigm. Judged distance as a function of target distance averaged across eight subjects. Error bars represent $1 \mathrm{SE}$. 


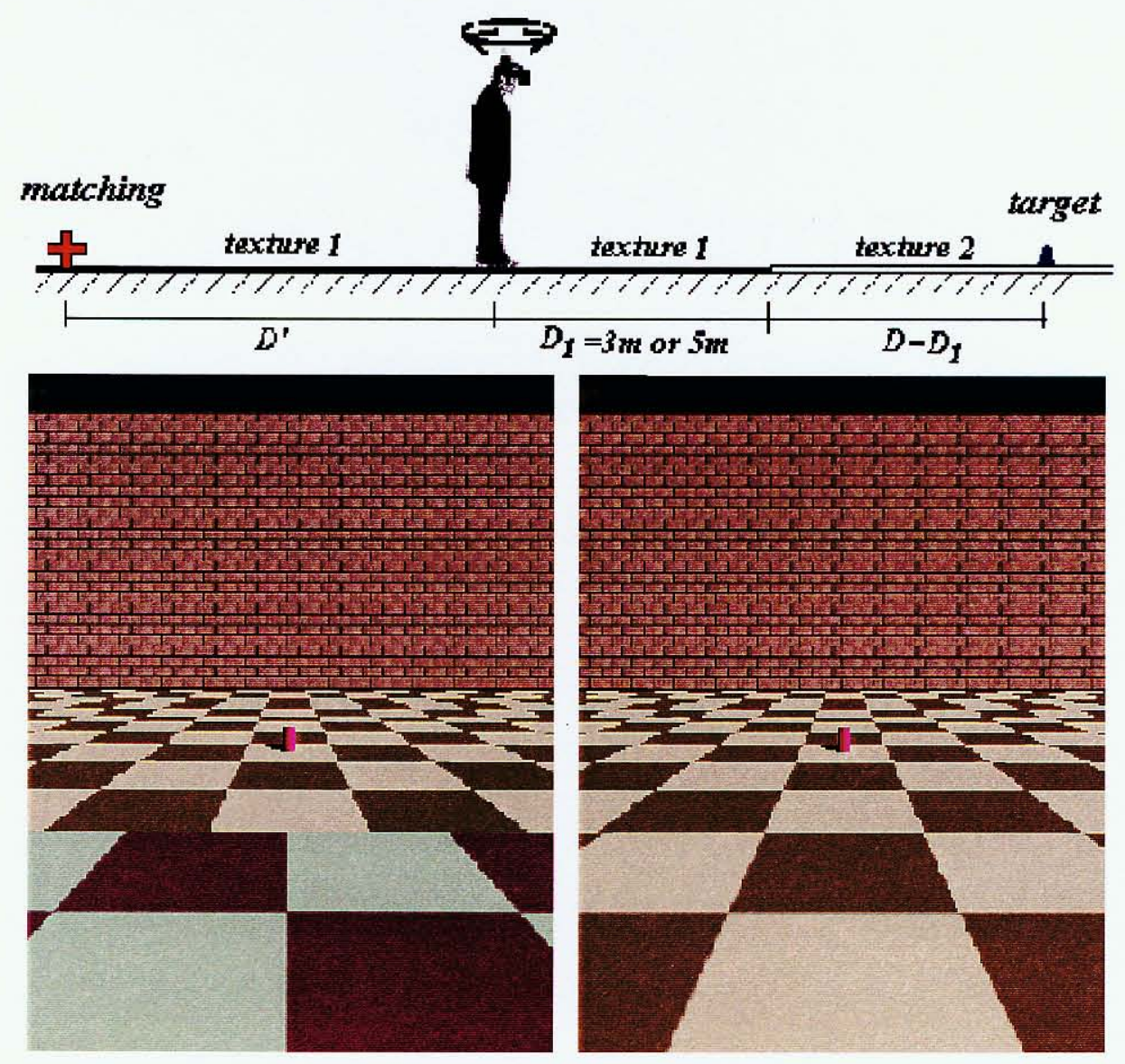

Figure 5.3. Distance underestimation due to the discontinuity in the ground texture. The ground was divided into two regions by differently-colored checkerboard patterns that were identical in spatial structure but displaced with $90^{\circ}$ phase shift. 

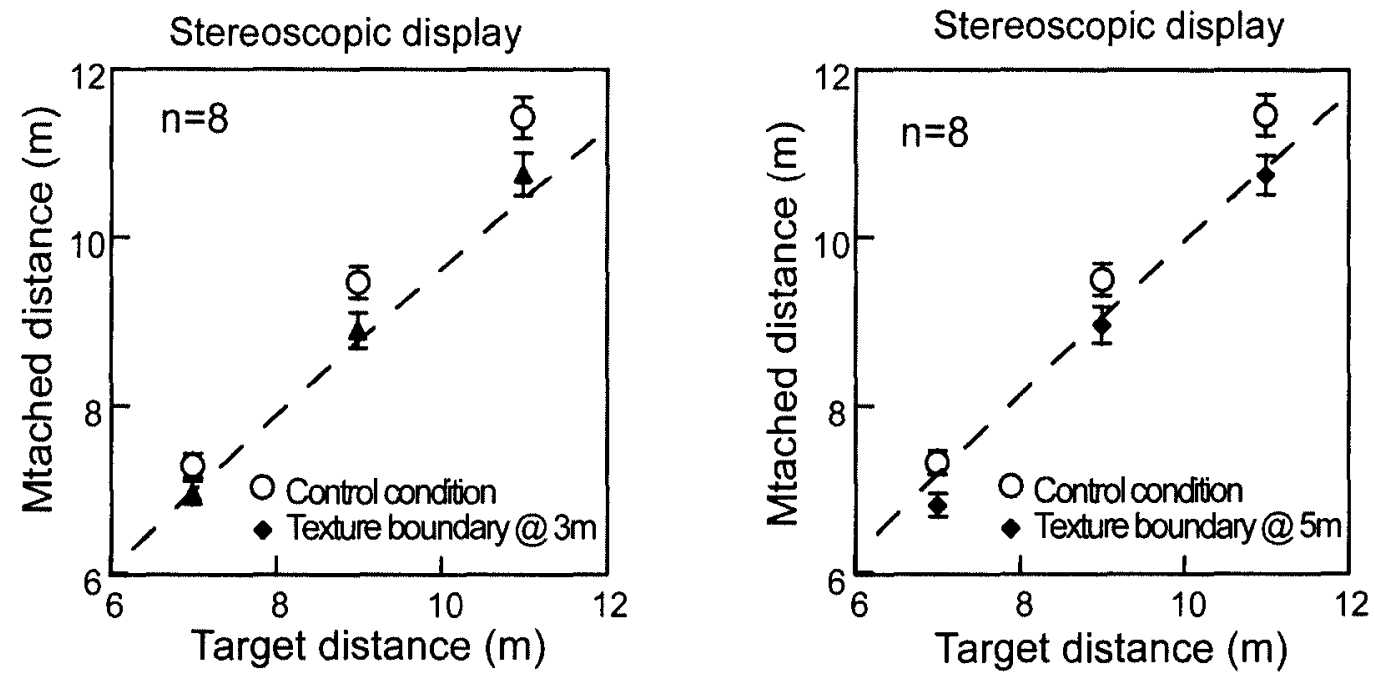

Figure 5.4. Distance underestimation in the presence of texture discontinuity. Distance judgment as a function of target distance averaged across eight subjects. Error bars represent $1 \mathrm{SE}$. The dashed line represents the prediction from Equation 4.3 using the perceived slants in Figure 5.5(b). 


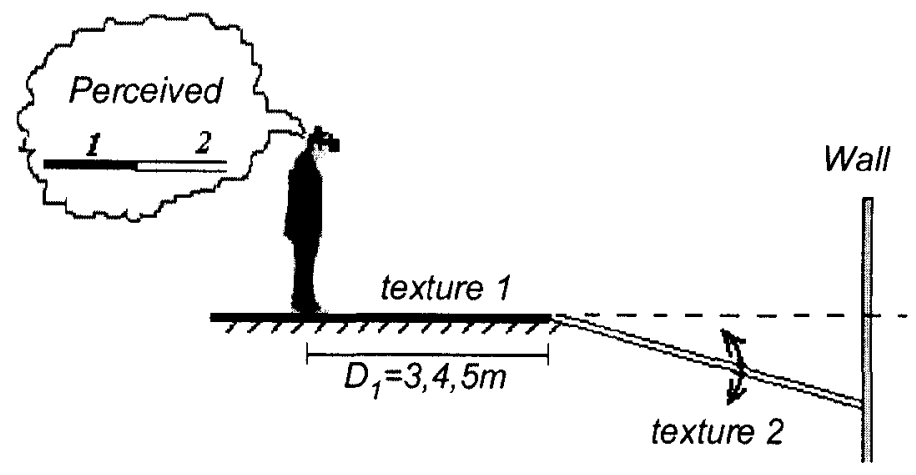

(a)

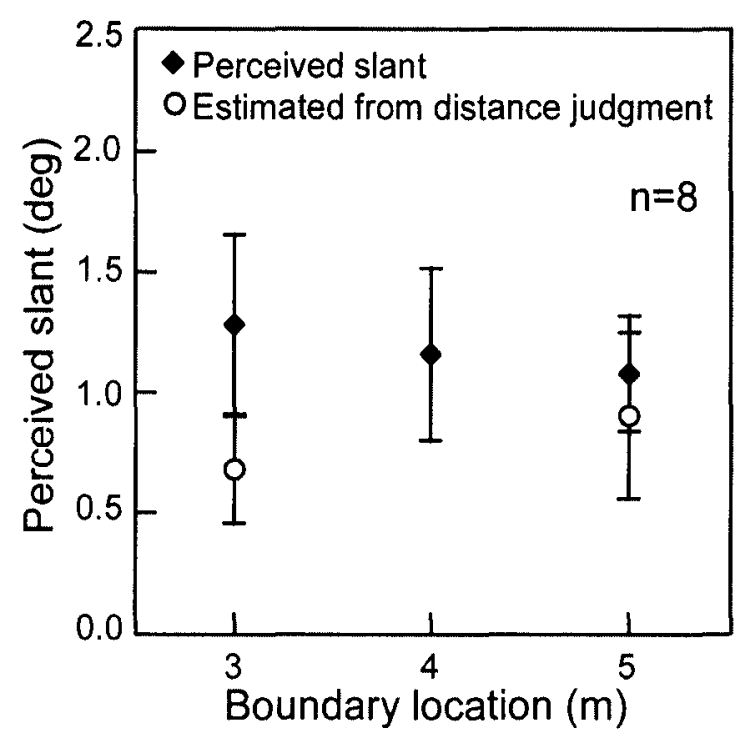

(b)

Figure 5.5. The "frontal shift" error in representing the ground due to texture discontinuity. (a). Errors in representing the ground surface were assessed using the slant-adjustment task described in the text. (b). Perceived slant as a function of the location of texture boundary averaged across eight subjects. Error bars represent $1 \mathrm{SE}$. Open circles denote the ground representation error estimated from distance judgments. 

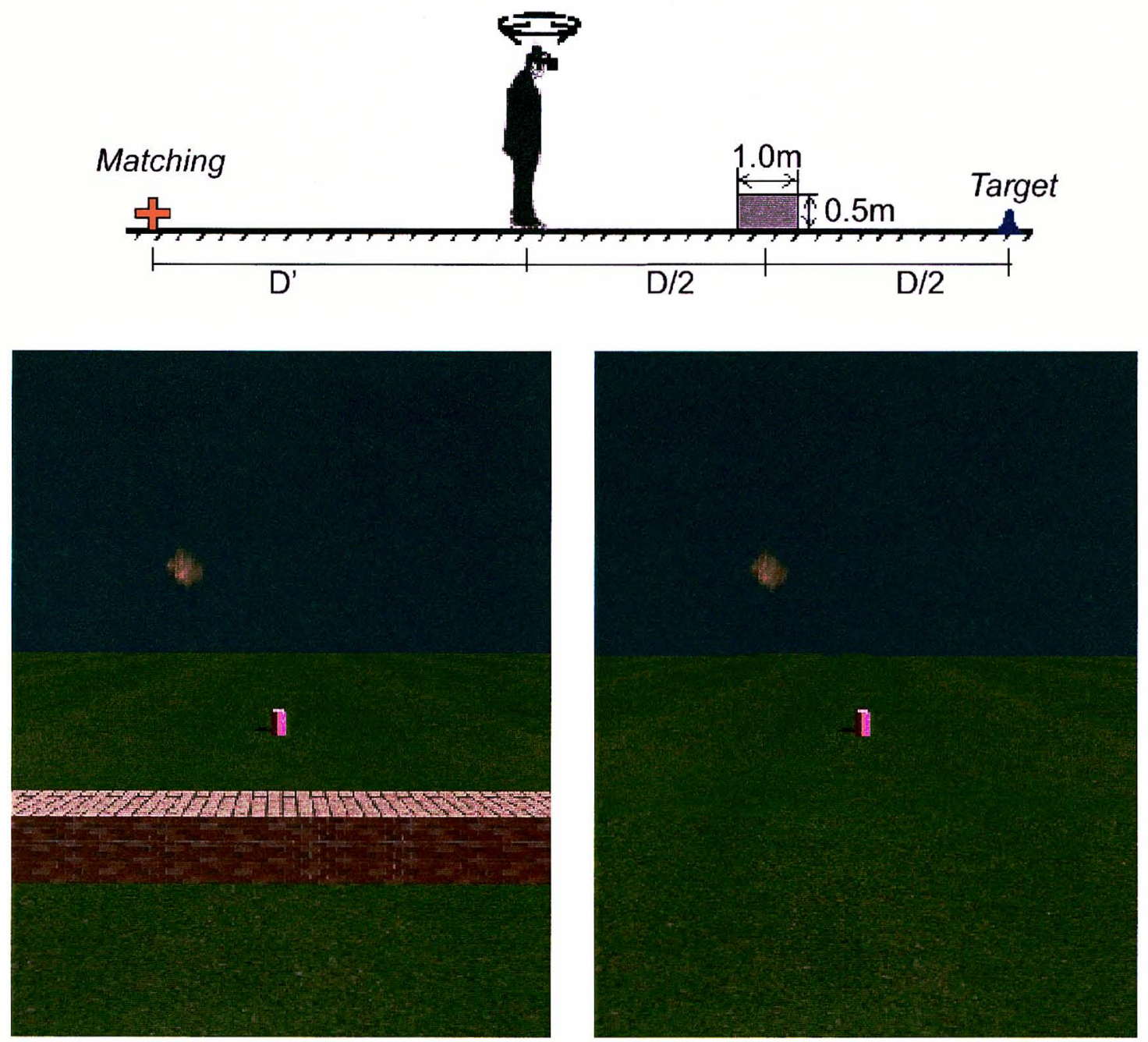

Figure 5.6. The settings and screen snapshots of the occlusion experiment.

From snapshots, the reader can see the occlusion effect: the distance from the bottom of the picture to the rod looks shorter in the left picture than that in the right picture. 

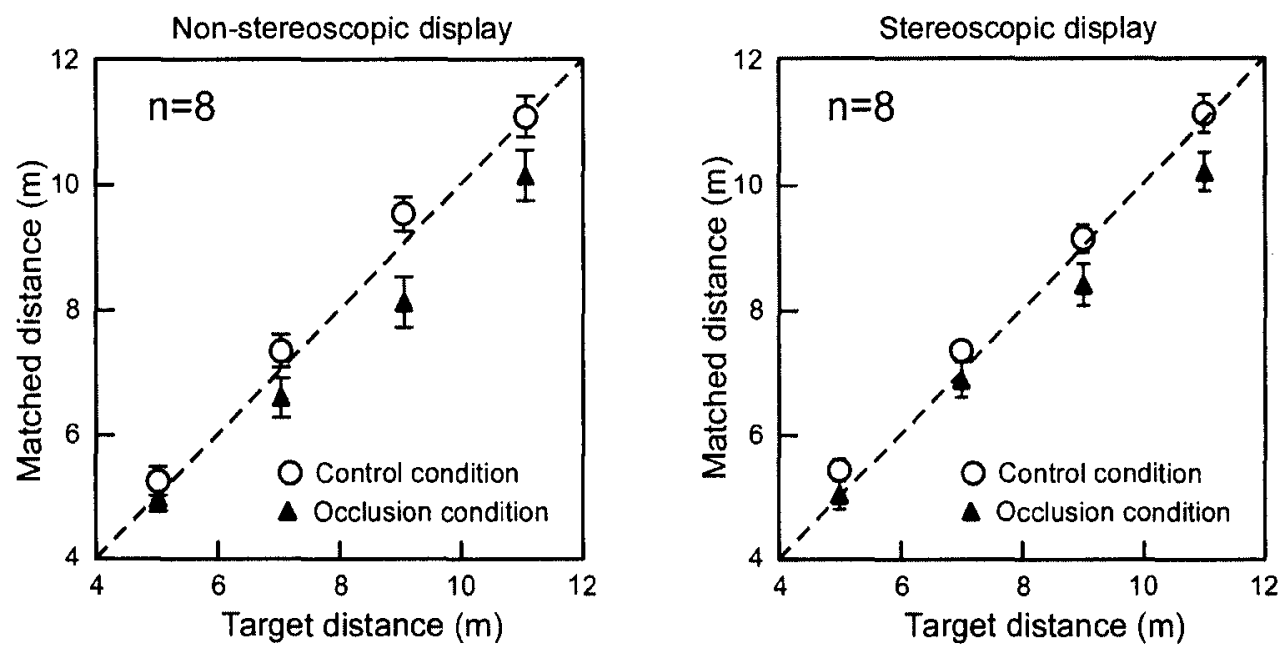

Figure 5.7. Distance underestimation caused by the occlusion of the ground.

The mean perceived distance is plotted as a function of the actual distance of the target. Error bars represent $1 \mathrm{SE}$. 


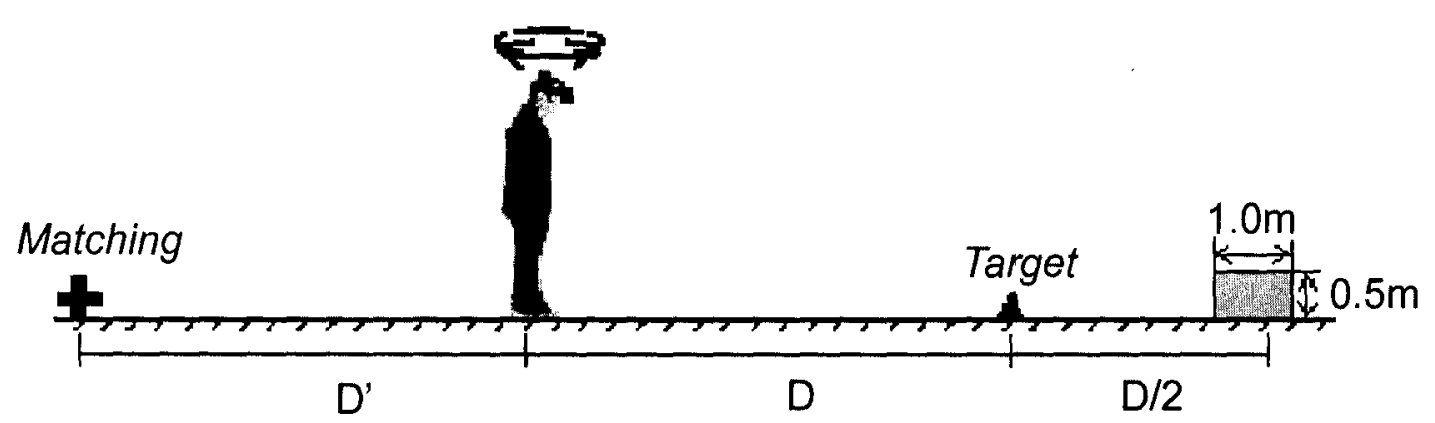

(a)

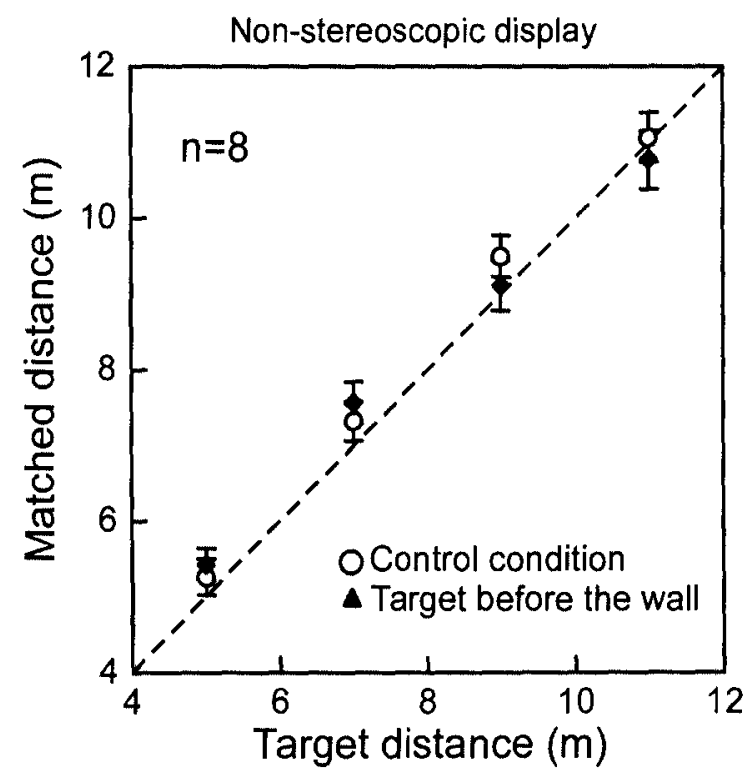

(b)

Figure 5.8 The control experiment to assess the possibility of "equidistance tendency". (a). The setting of the experiment. (b). Judged distance as a function of target distance averaged across eight subjects. When the wall was placed behind the target, distance perception was little affected by it if a continuous ground was visible. Error bars represent $1 \mathrm{SE}$. 


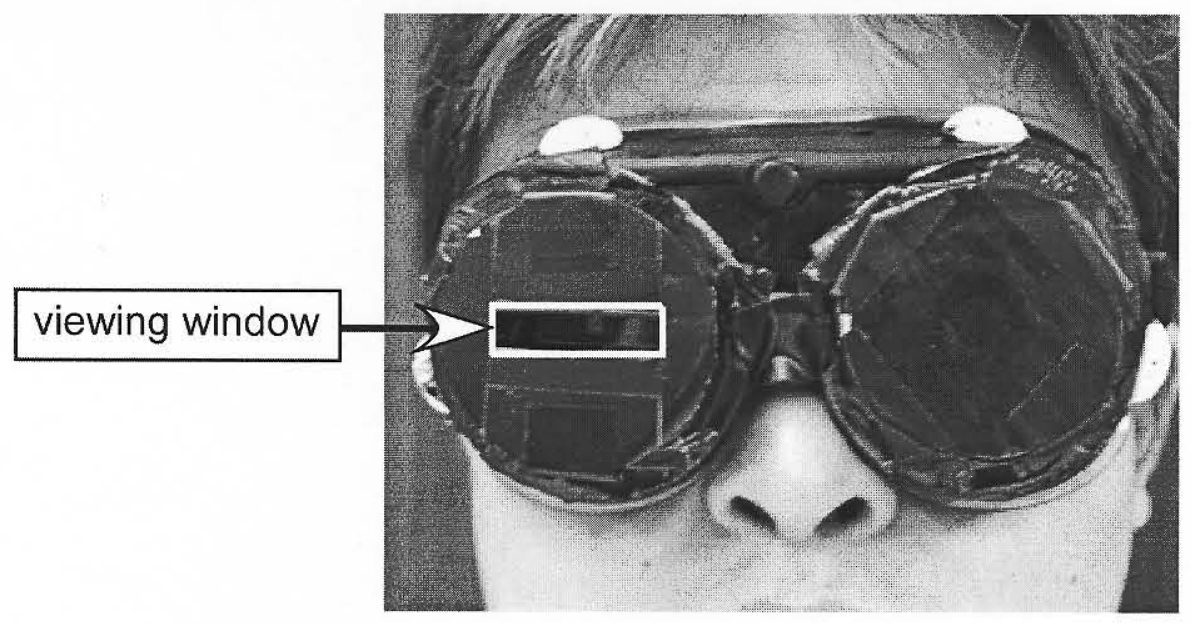

Figure 6.1. The FOV-restricting goggles, which was used to limit the observer's field of view (FOV) to a particular size and shape. 


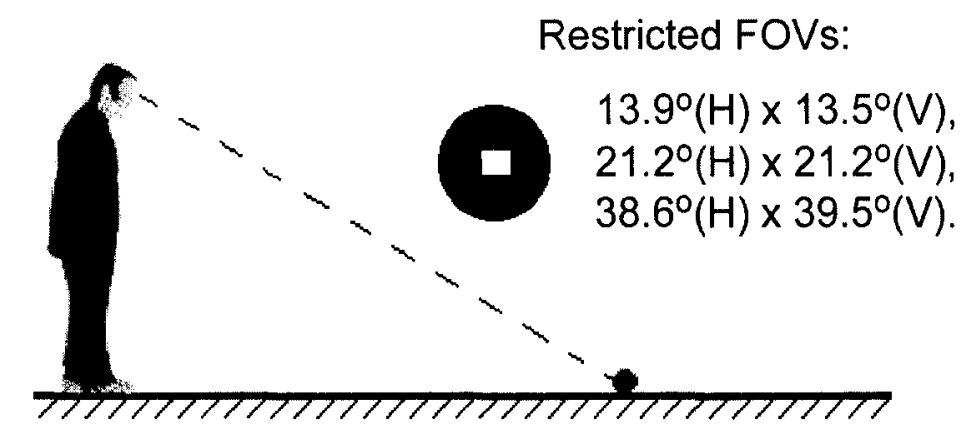

(a)

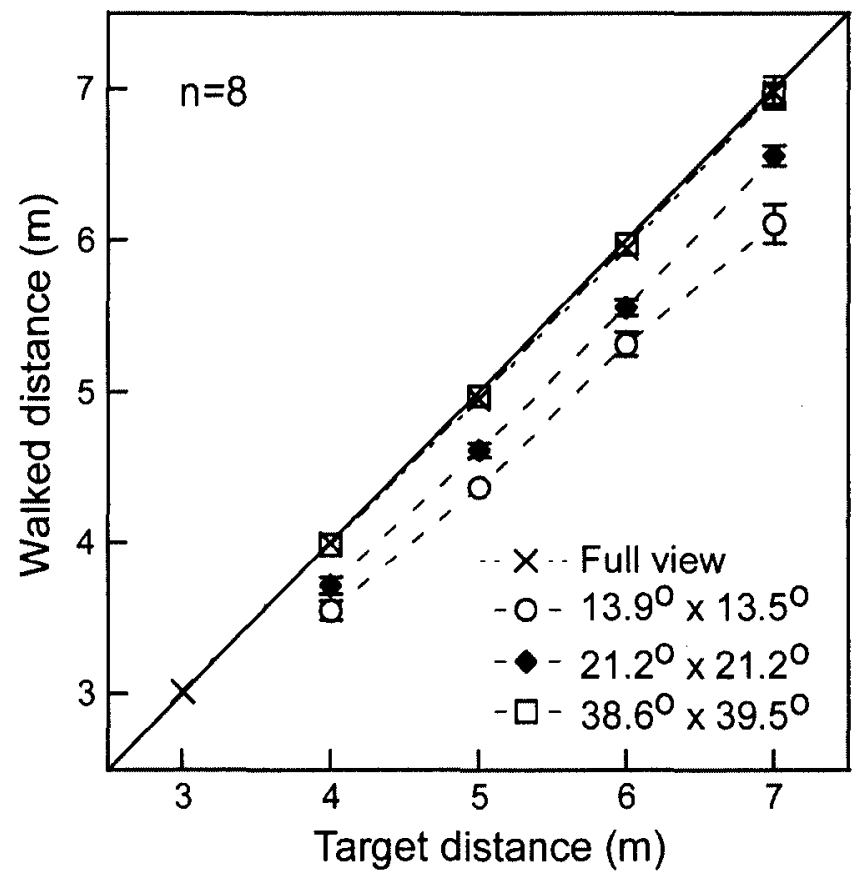

(b)

Figure 6.2. Absolute distance judgment from a static view with restricted FOV. (a). The experimental settings for Exp. 6.1. Subjects' judgment of distance was measured by using the blindfolded walking paradigm. (b). Walked distance as a function of target distance averaged across eight subjects. Error bars represent $1 \mathrm{SE}$. 


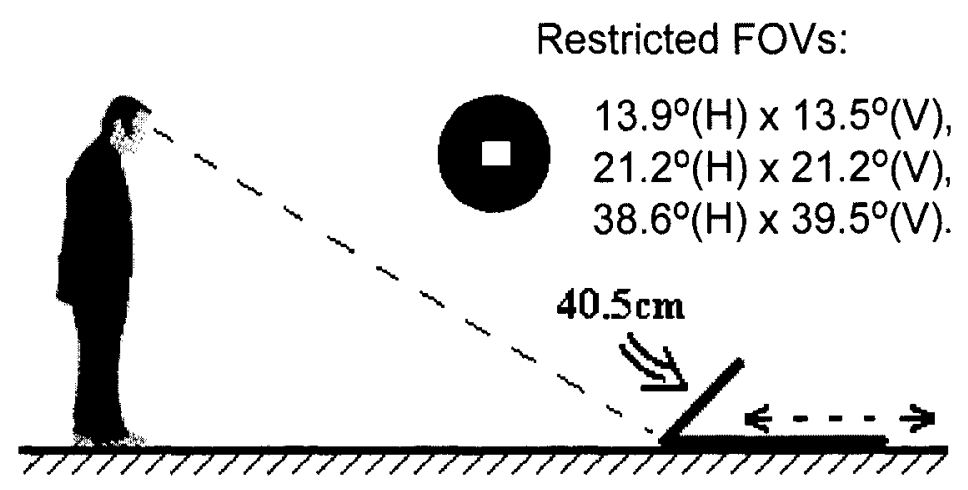

(a)

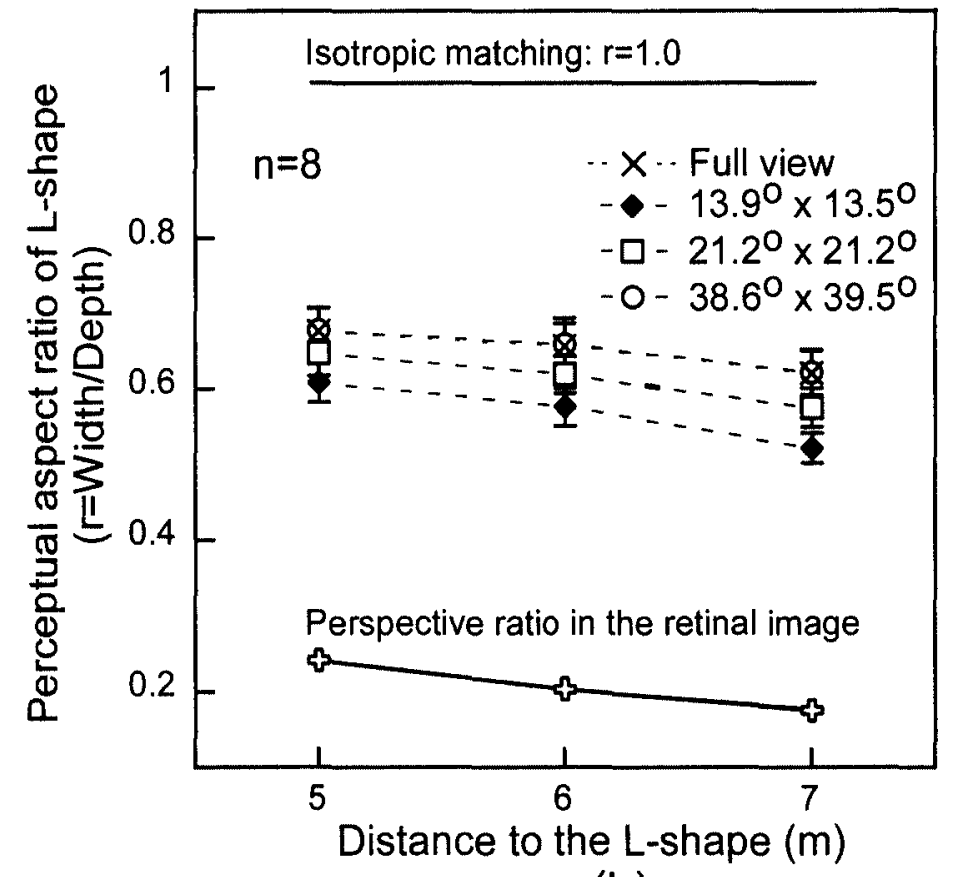

(b)

Figure 6.3. Relative distance judgment from a static view with restricted FOV.

(a). Settings of the experiment. Relative distance perception was measured by using a perceptual matching task. Subjects were asked to adjust the L-shape's depth arm until both arms were judged as being of the same physical length. (b). The perceptual aspect ratio of the L-shape ( $r$-width/depth) as a function of the L-shape's location averaged across eight subjects. Error bars represent $1 \mathrm{SE}$. 


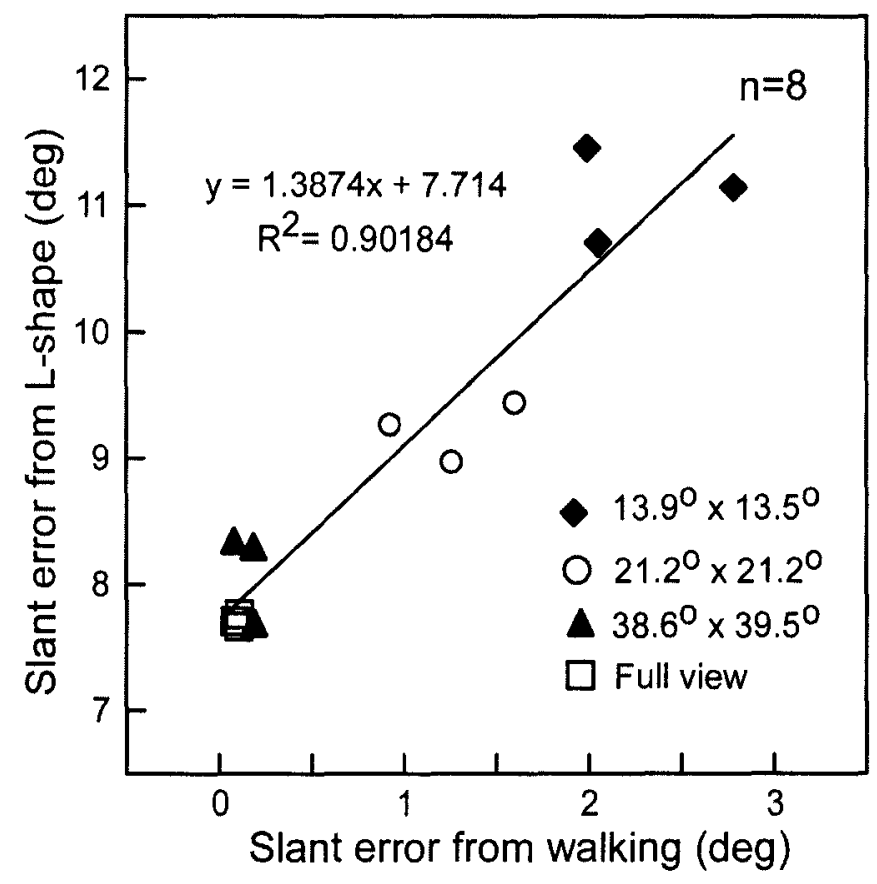

Figure 6.4. Slant errors from the blindfolded walking \& L-shape matching tasks using Eq. 4.1 \& 4.2. For each location and FOV tested, the estimated slant errors from two tasks are correlated. 


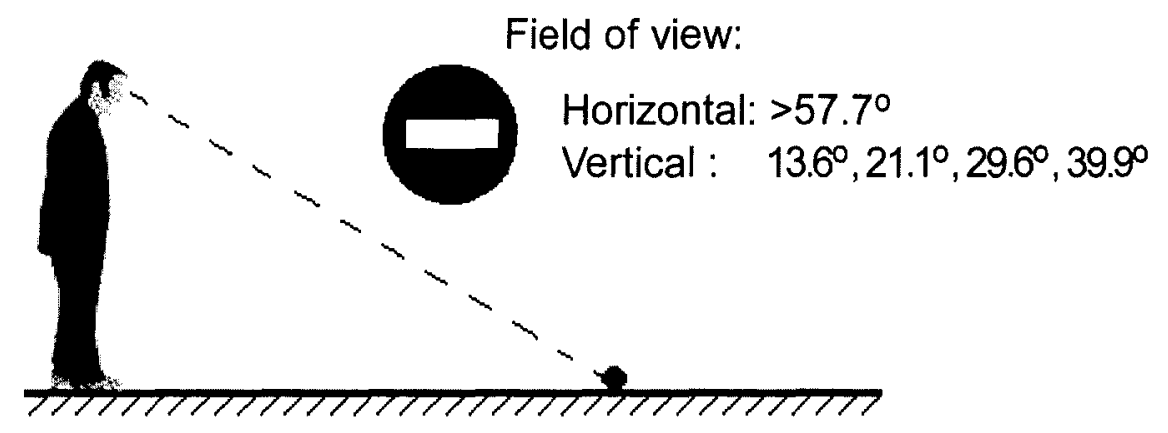

(a)

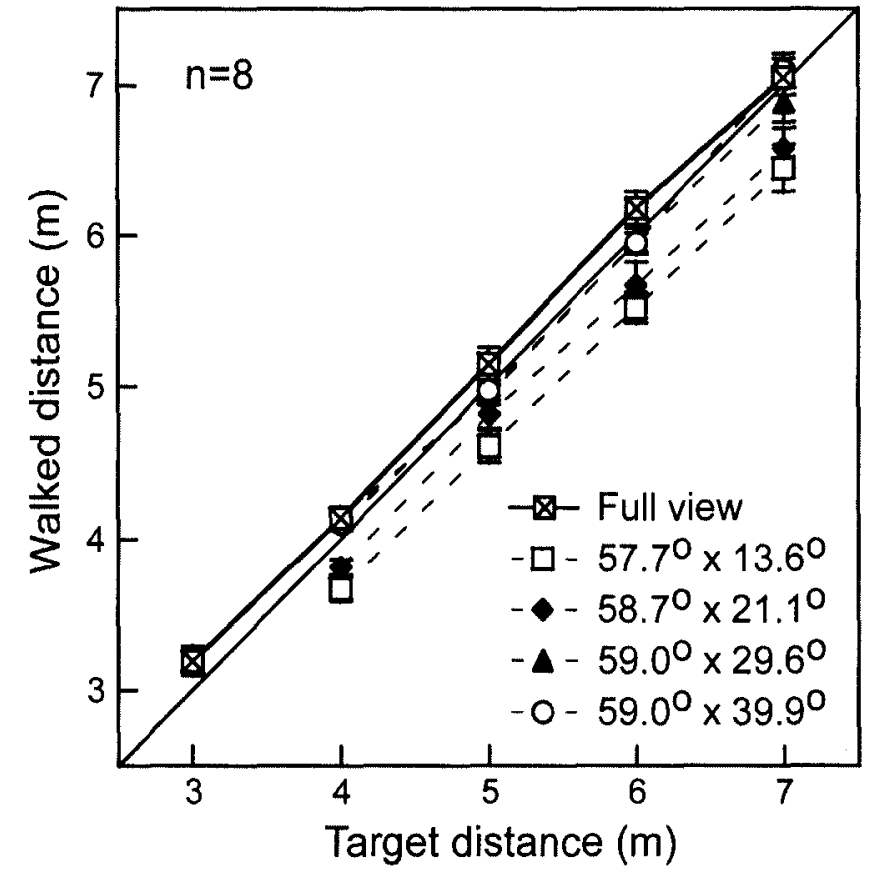

(b)

Figure 6.5. Distance judgment from a static view with vertically-restricted FOV. (a). The experimental settings for Exp. 6.3. Subjects' judgment of distance was measured by using the blindfolded walking paradigm. (b). Walked distance as a function of target distance averaged across eight subjects. Error bars represent $1 \mathrm{SE}$. 


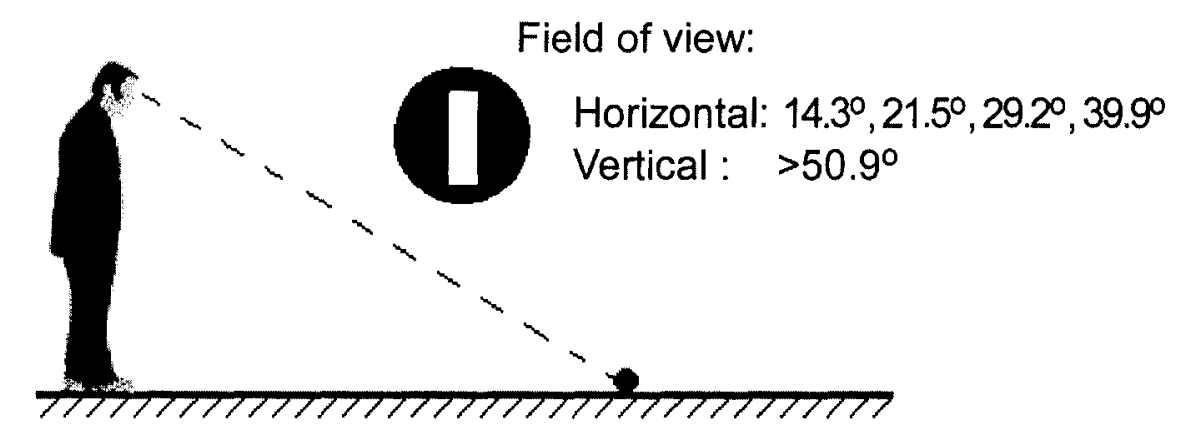

(a)

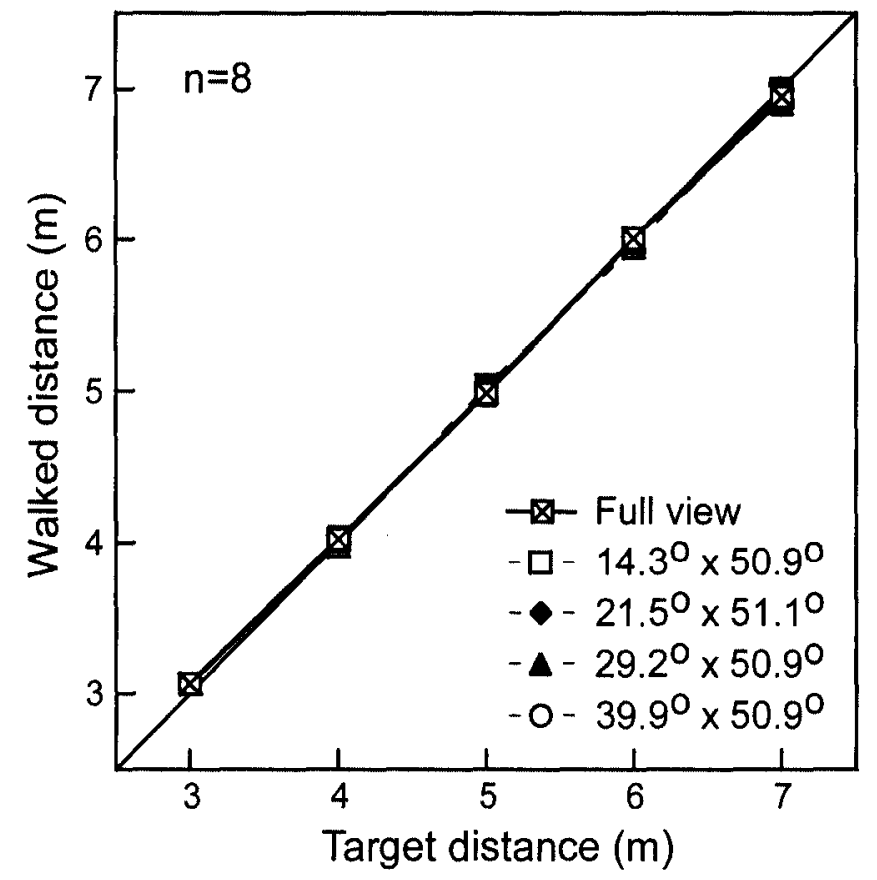

(b)

Figure 6.6. Distance judgment from a static view with horizontally-restricted FOV. (a). The experimental settings for Exp. 6.4. (b). Walked distance as a function of target distance averaged across eight subjects. Error bars represent $1 \mathrm{SE}$. 


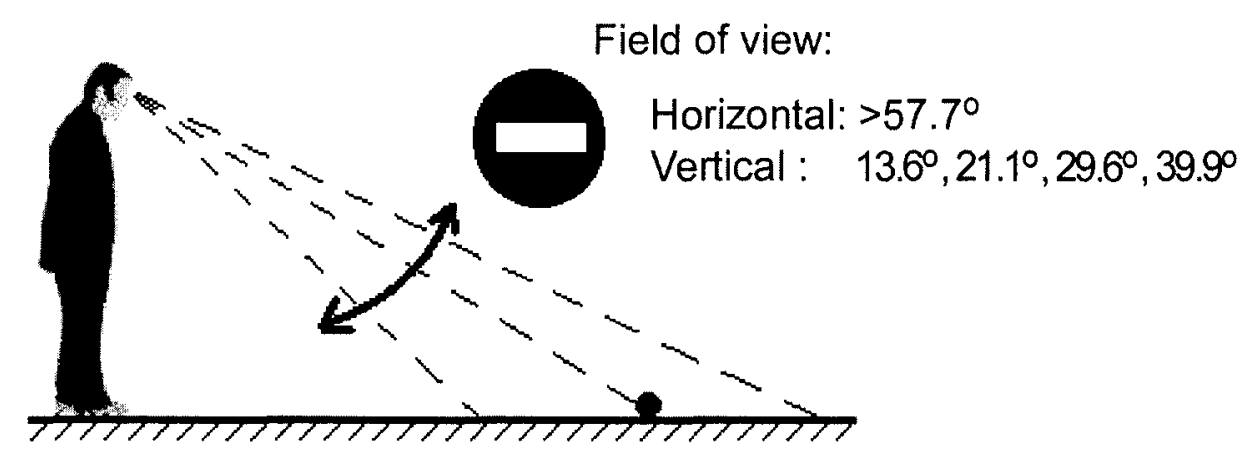

(a)

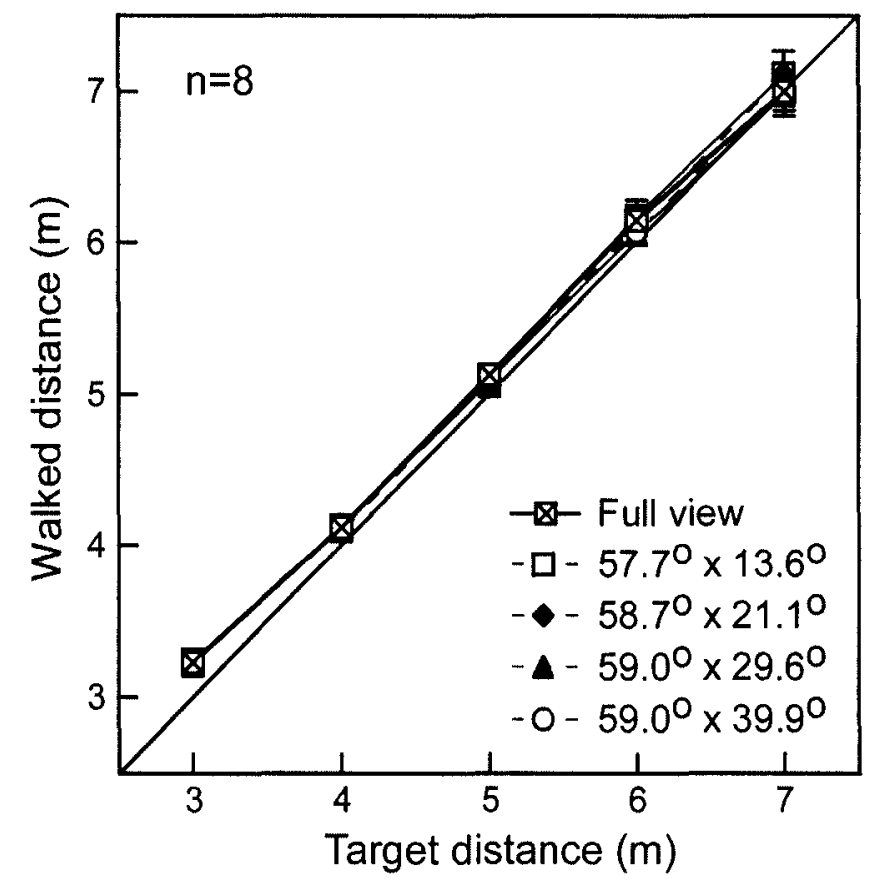

(b)

Figure 6.7. Distance judgment with free head motion. (a). The experimental settings for Exp. 6.5. (b). Walked distance as a function of target distance averaged across eight subjects. Error bars represent $1 \mathrm{SE}$. 

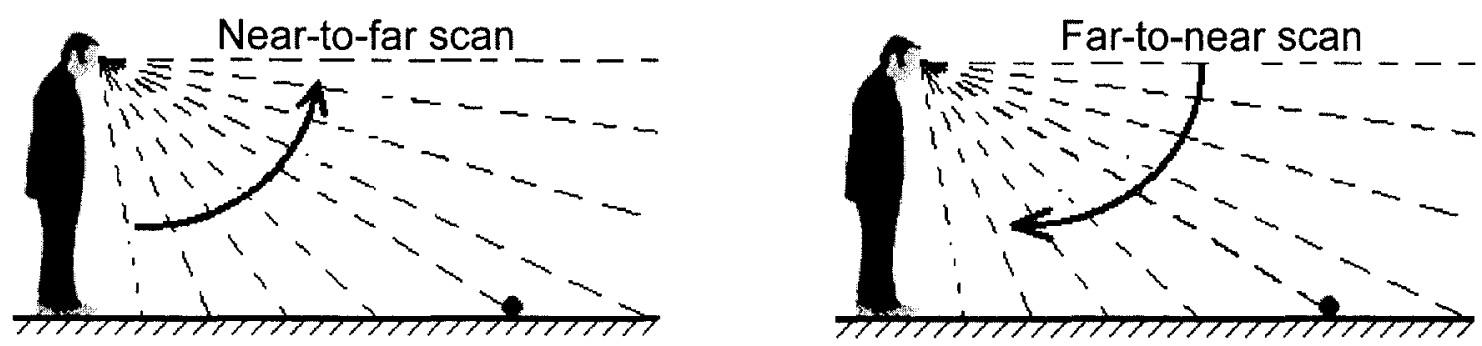

Field of view:

Horizontal: $>57.7^{\circ}$

Vertical : $13.6^{\circ}, 21.1^{\circ}$

(a)

FOV: $57.7^{\circ} \times 13.6^{\circ}$

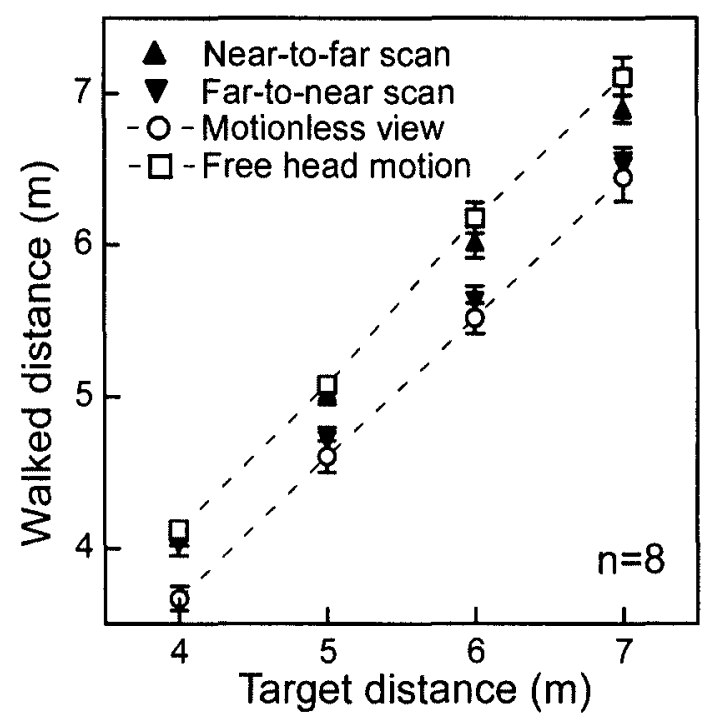

FOV: $57.7^{\circ} \times 21.1^{\circ}$

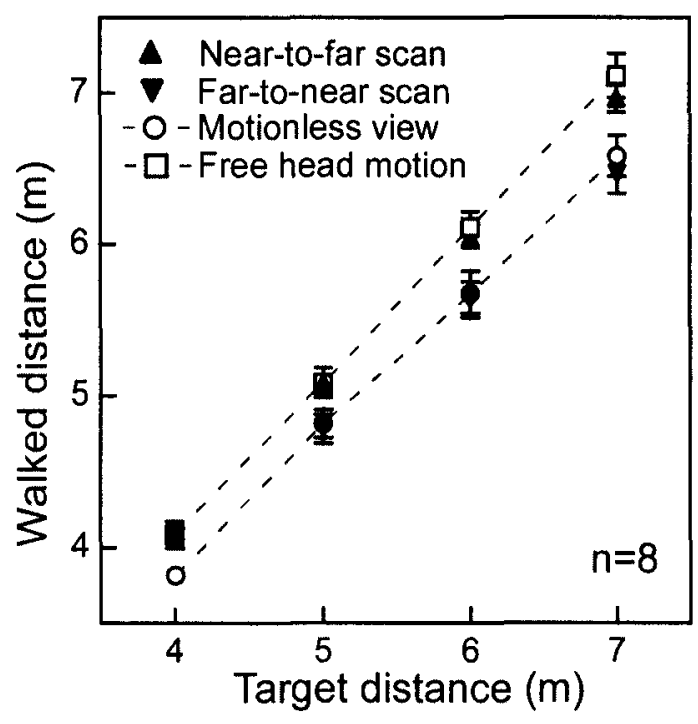

(b)

Figure 6.8. The direction asymmetry in distance judgment. (a). Settings of Exp 6.6. (b). Walked distance as a function of target distance averaged across eight subjects. Error bars represent $1 \mathrm{SE}$. 

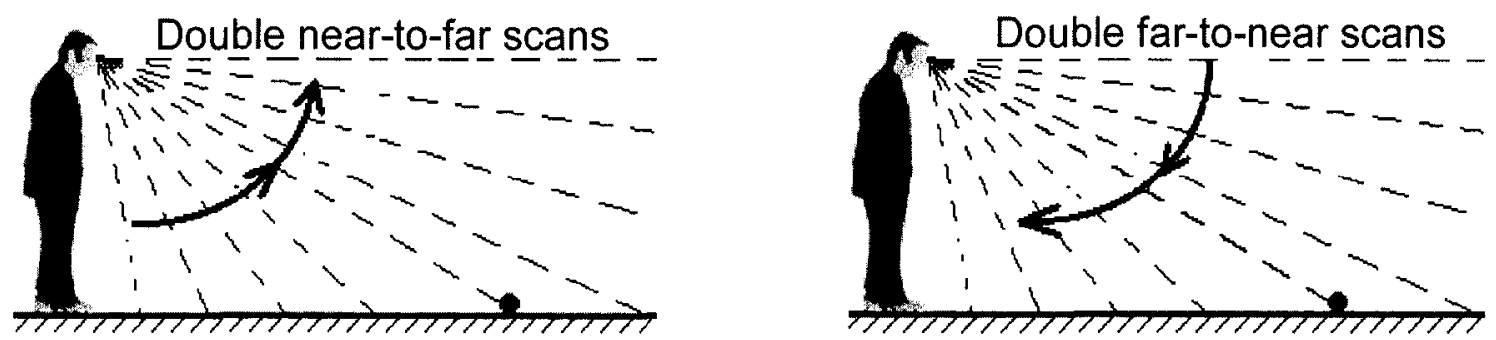

Field of view:

Horizontal: $>57.7^{\circ}$

Vertical : $13.6^{\circ}, 21.1^{\circ}$

(a)
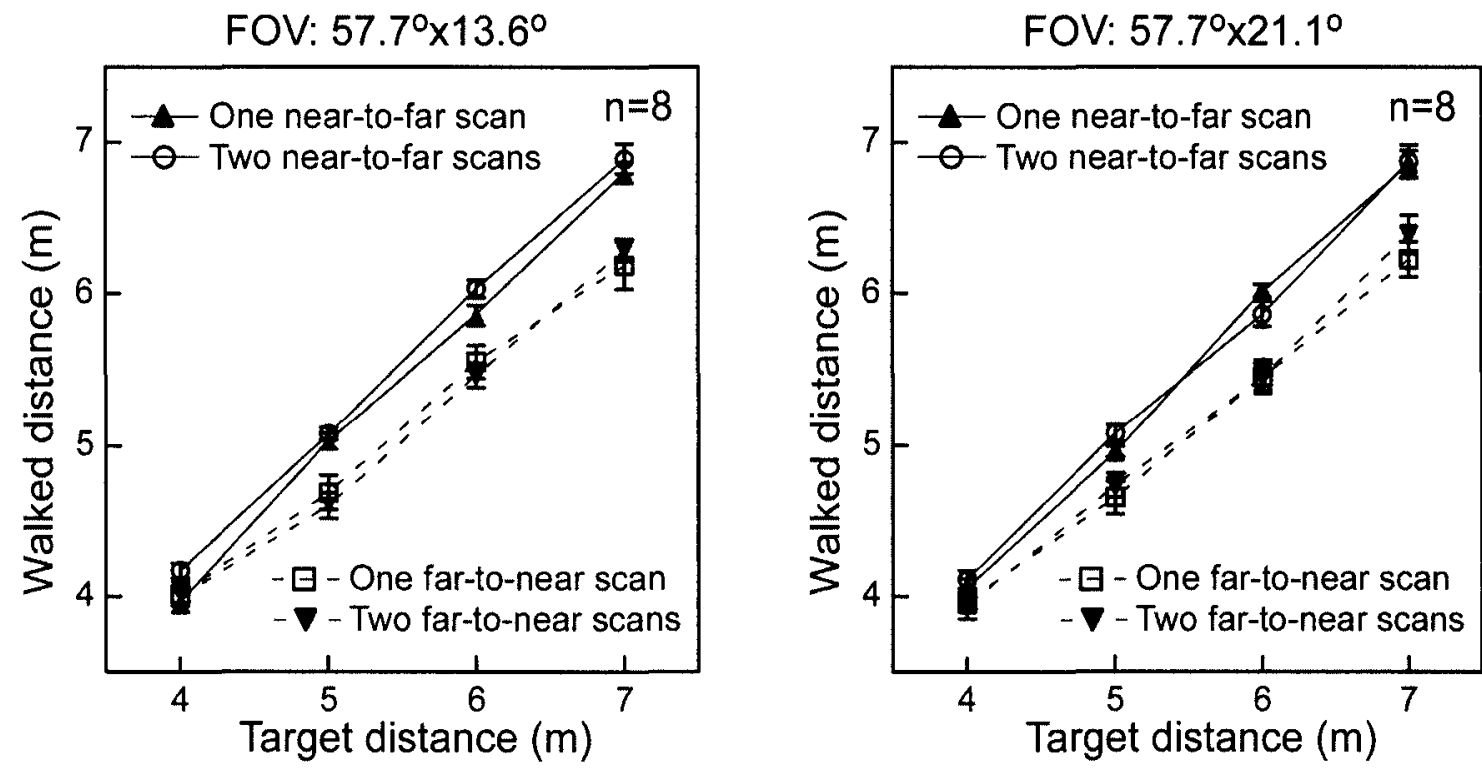

(b)

Figure 6.9. Distance judgment with two directional scans. (a). Settings of

Exp. 6.7. (b). Walked distance as a function of target distance averaged across eight subjects. Error bars represent $1 \mathrm{SE}$ 

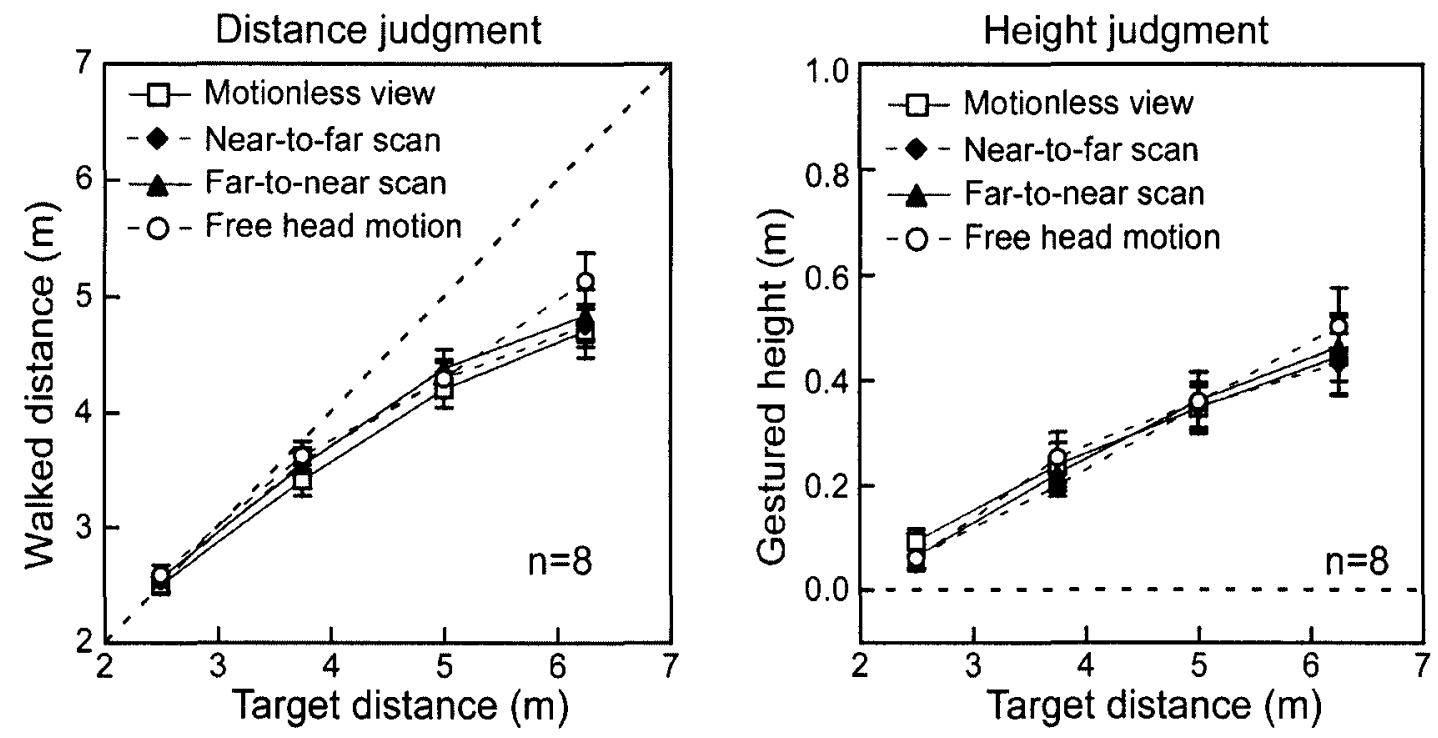

(a)

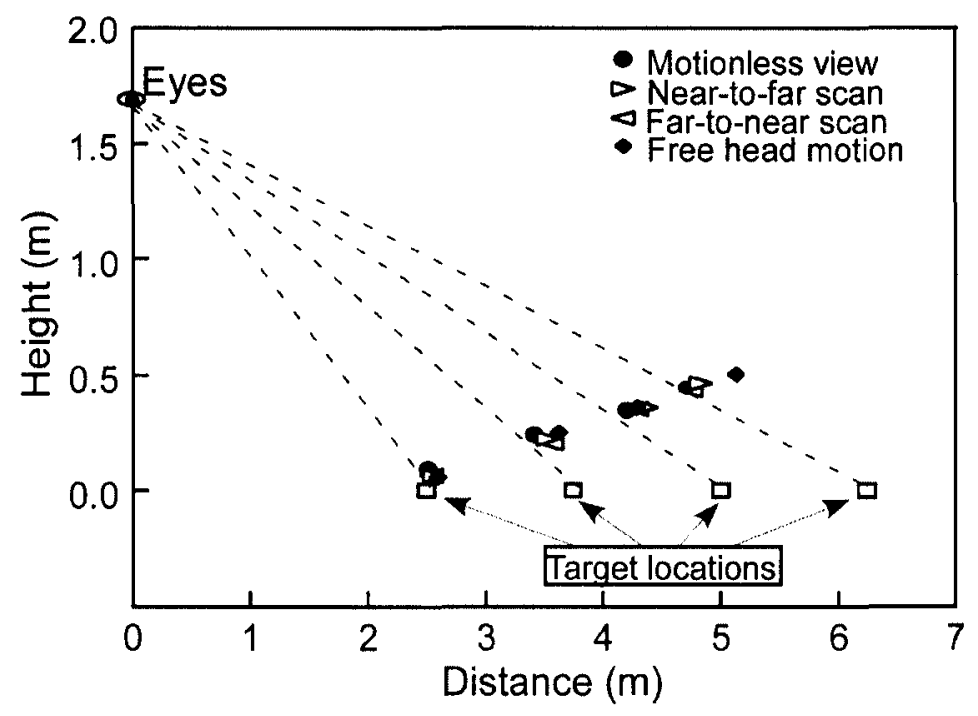

(b)

Figure 6.10. The control experiment in the dark. (a). Walked distance and gestrued height as functions of target distance averaged across eight subjects. Error bars represent 1 SE. (b). Perceived locations of the target with or without head motion in the dark. Clearly, the target's location was misperceived in all four conditions, although the angular declination of the target was accurately perceived. 

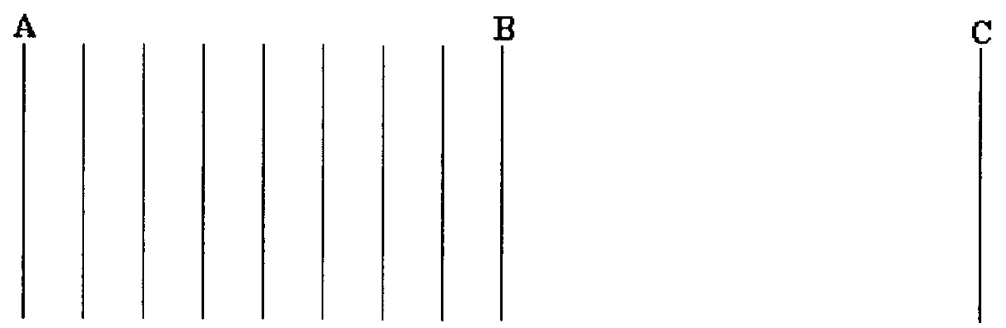

Figure 7.1. The Oppel-Kundt illusion. An unfilled space (B-C) looks smaller than a filled space (A-B). 

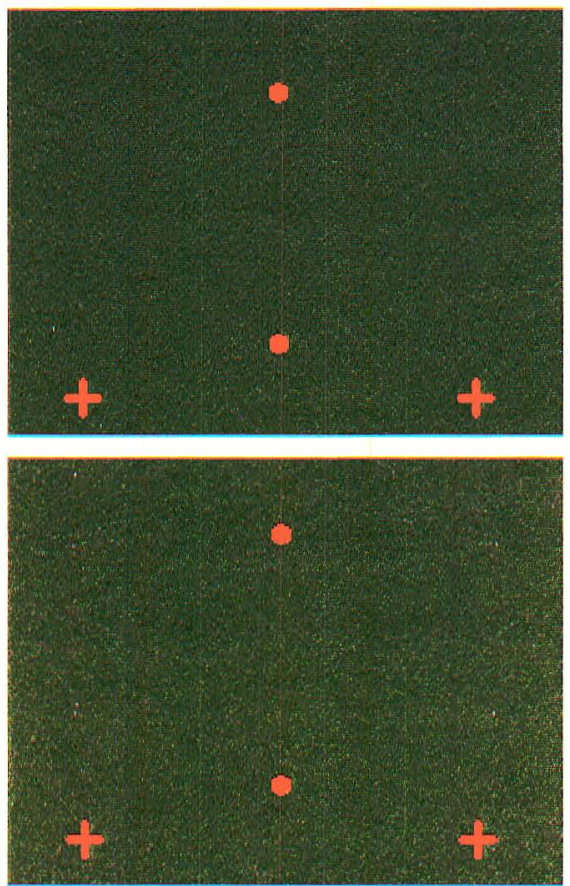

(a)

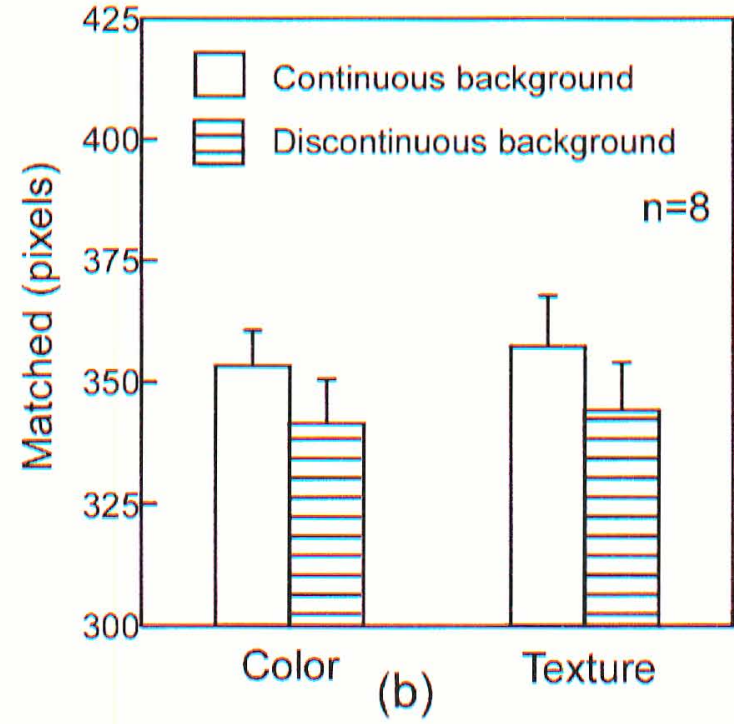

Figure 7.2. The texture-discontinuity effect in a frontoparallel plane. (a). The screen snapshots of the stimuli. (b). The mean judged distance from 8 subjects. Error bars represent $1 \mathrm{SE}$. 

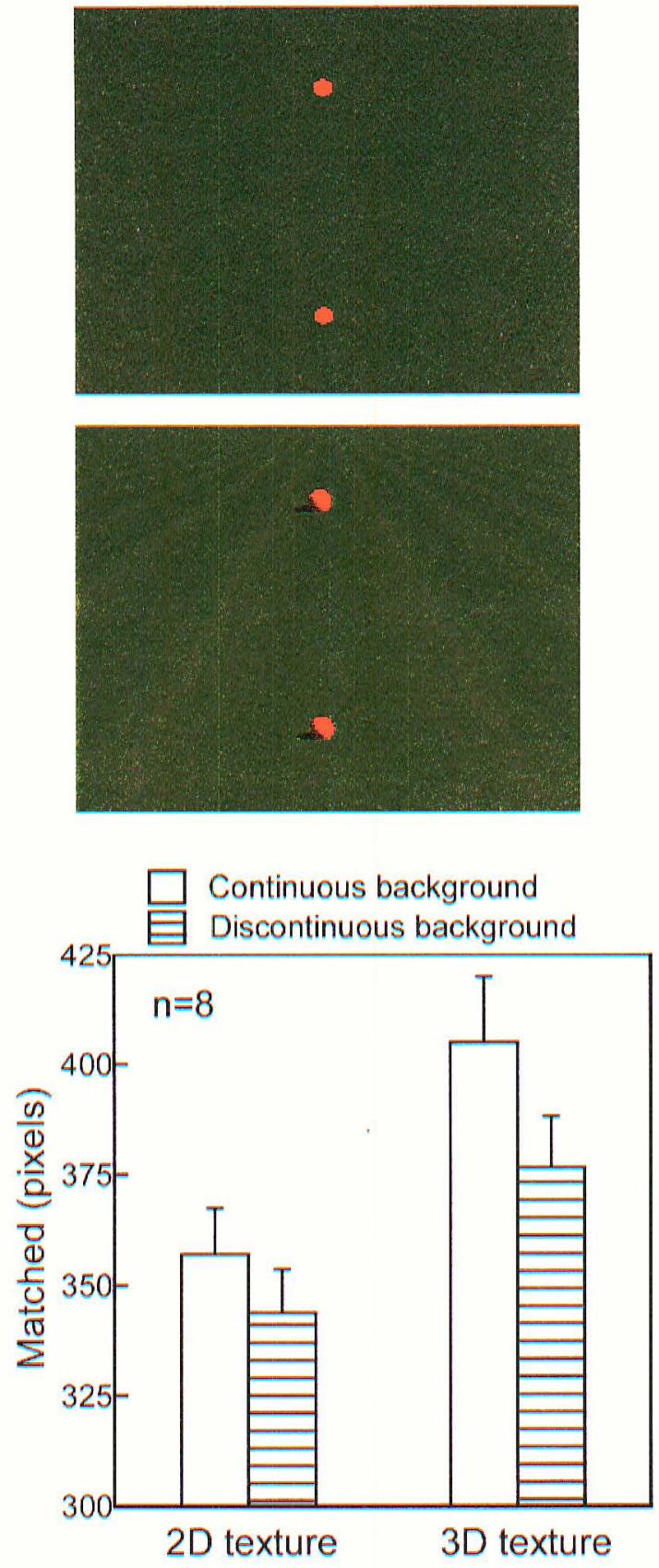

(b)

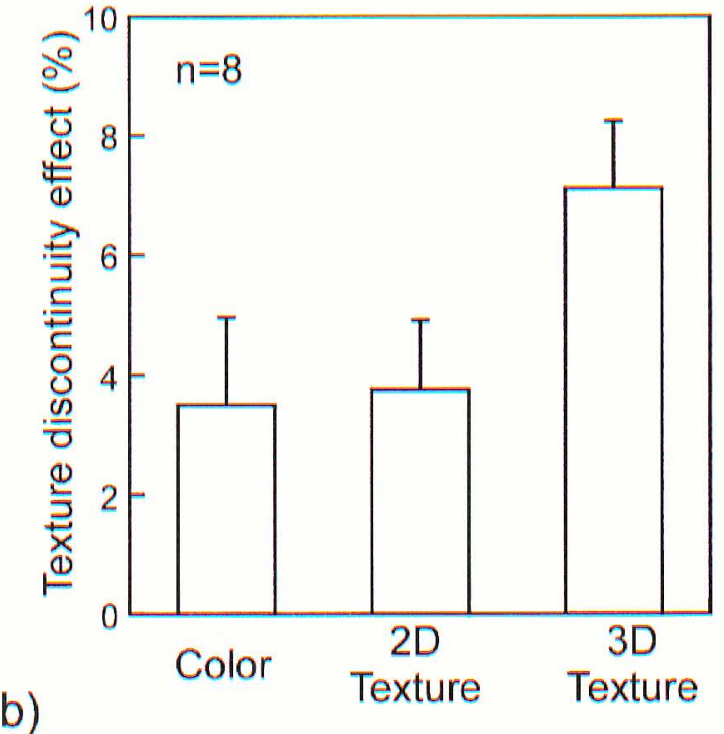

(a)

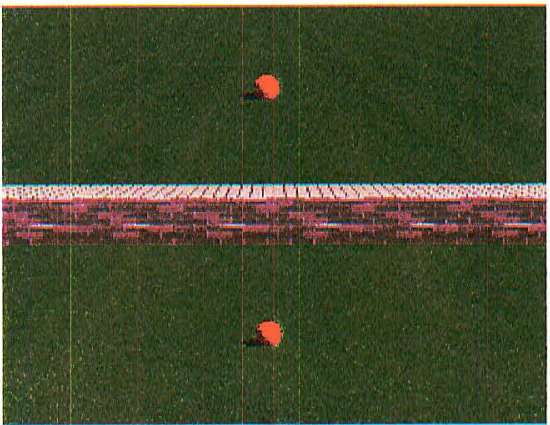

Figure 7.3. The influence of perspective information on the discontinuity effect.

(a). The screen snapshots of the stimuli. (b). Left: the mean matched distance from 8 subjects. Right: the discontinuity effect as a percentage of the judged distance. Error bars represent $1 \mathrm{SE}$. 

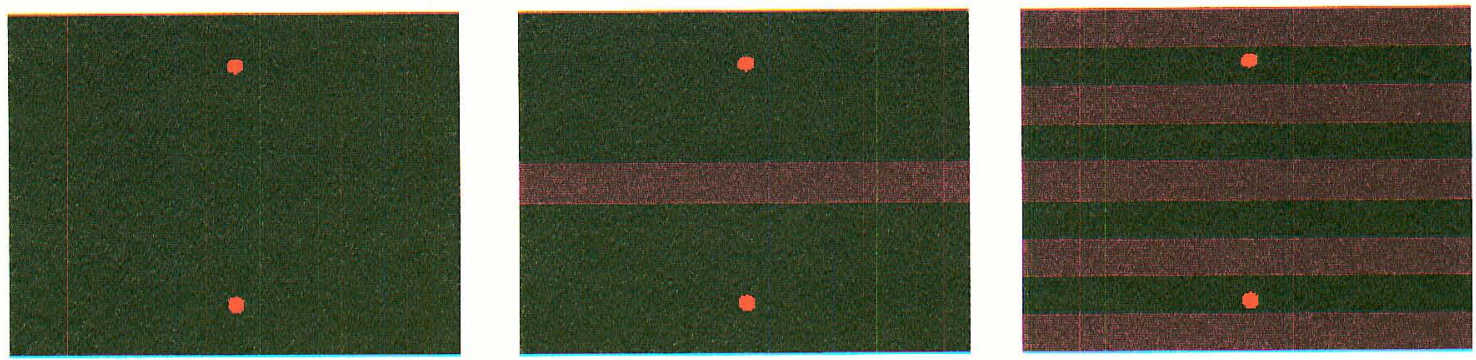

(a)
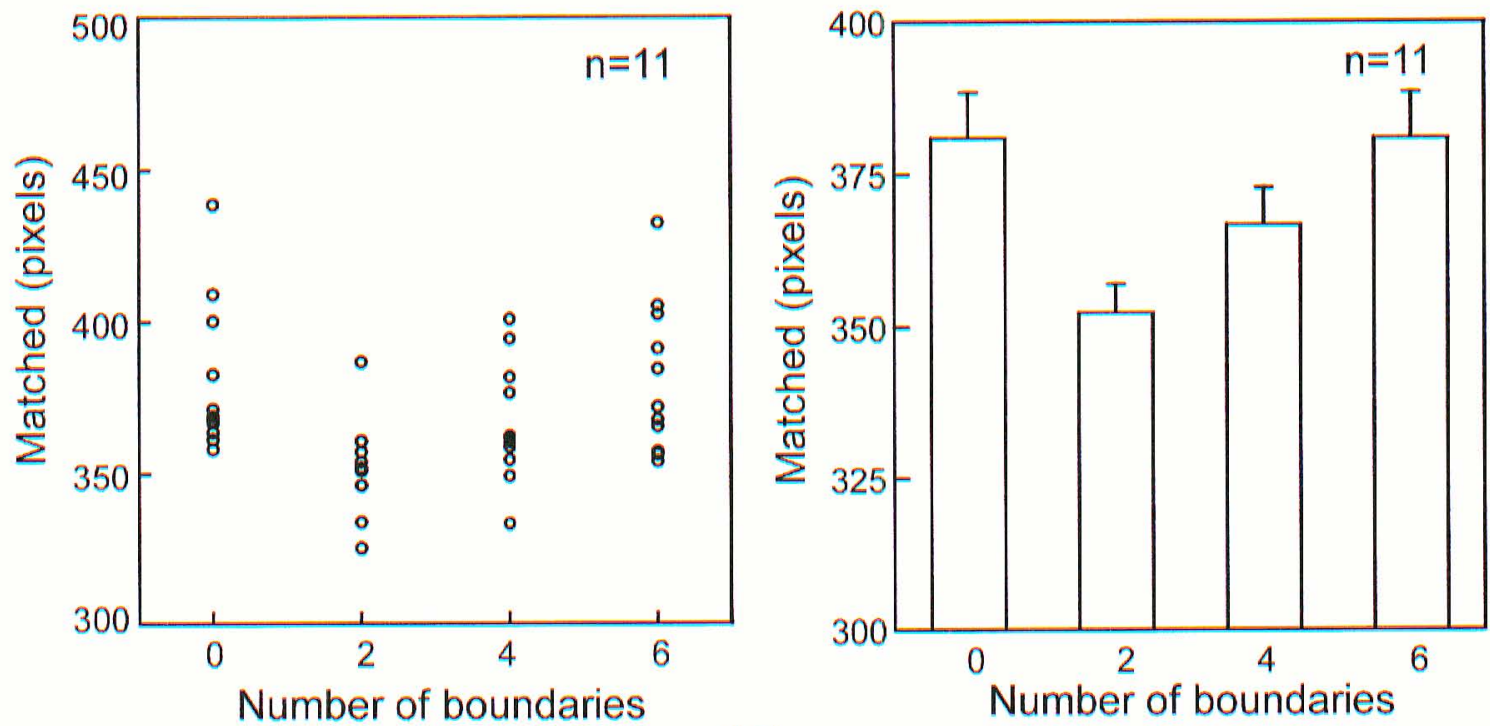

(b)

Figure 7.4. Distance judgment with different number of boundaries. (a). The screen snapshots of the stimuli. (b). Left: subjects' judgments of distance in four conditions. Right: the effect as a percentage of the mean judged distance. Error bars represent $1 \mathrm{SE}$. 


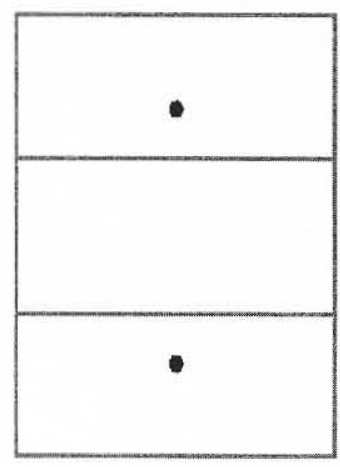

(a)

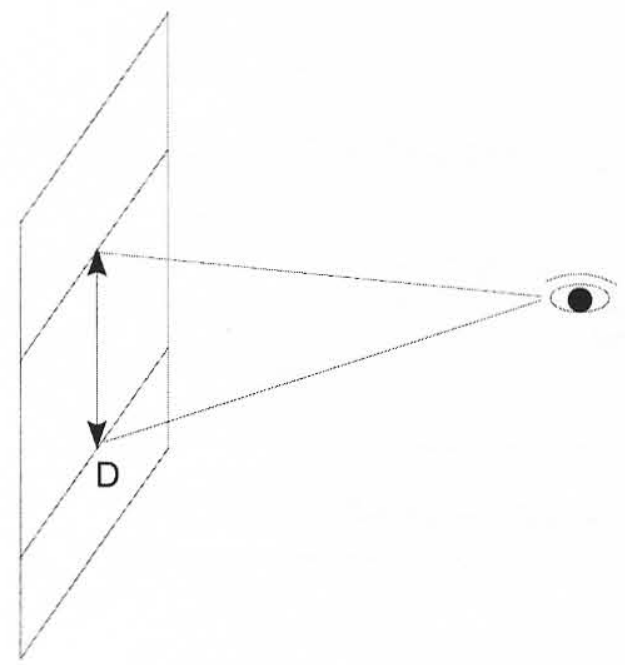

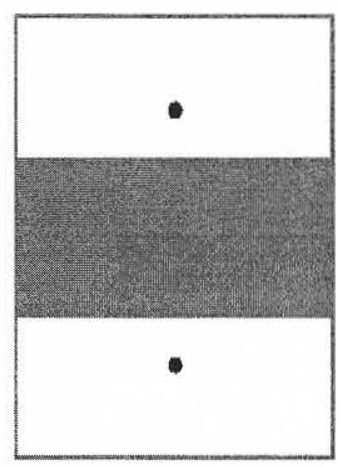

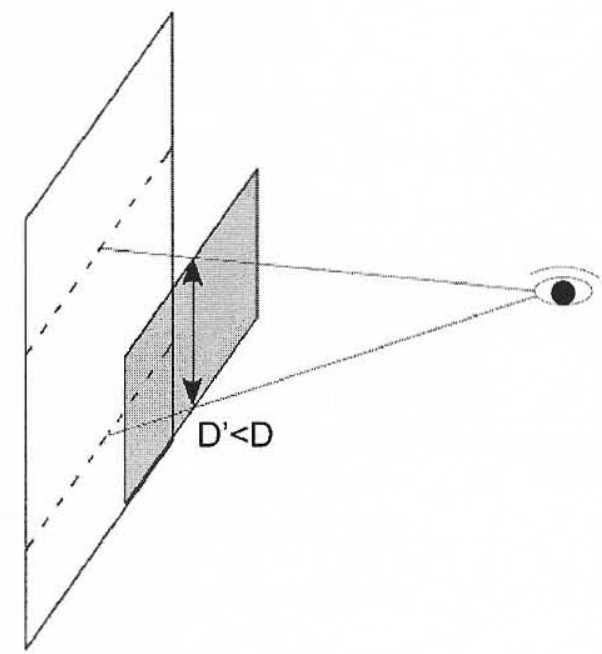

(b)

Figure 7.5. A possible explanation for the texture-discontinuity effect in frontoparallel plane. 

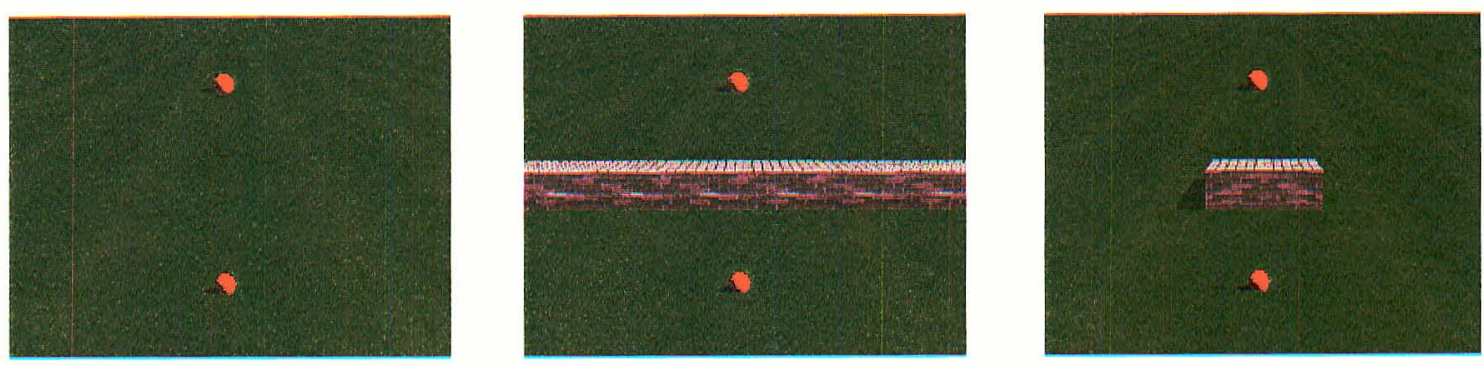

(a)
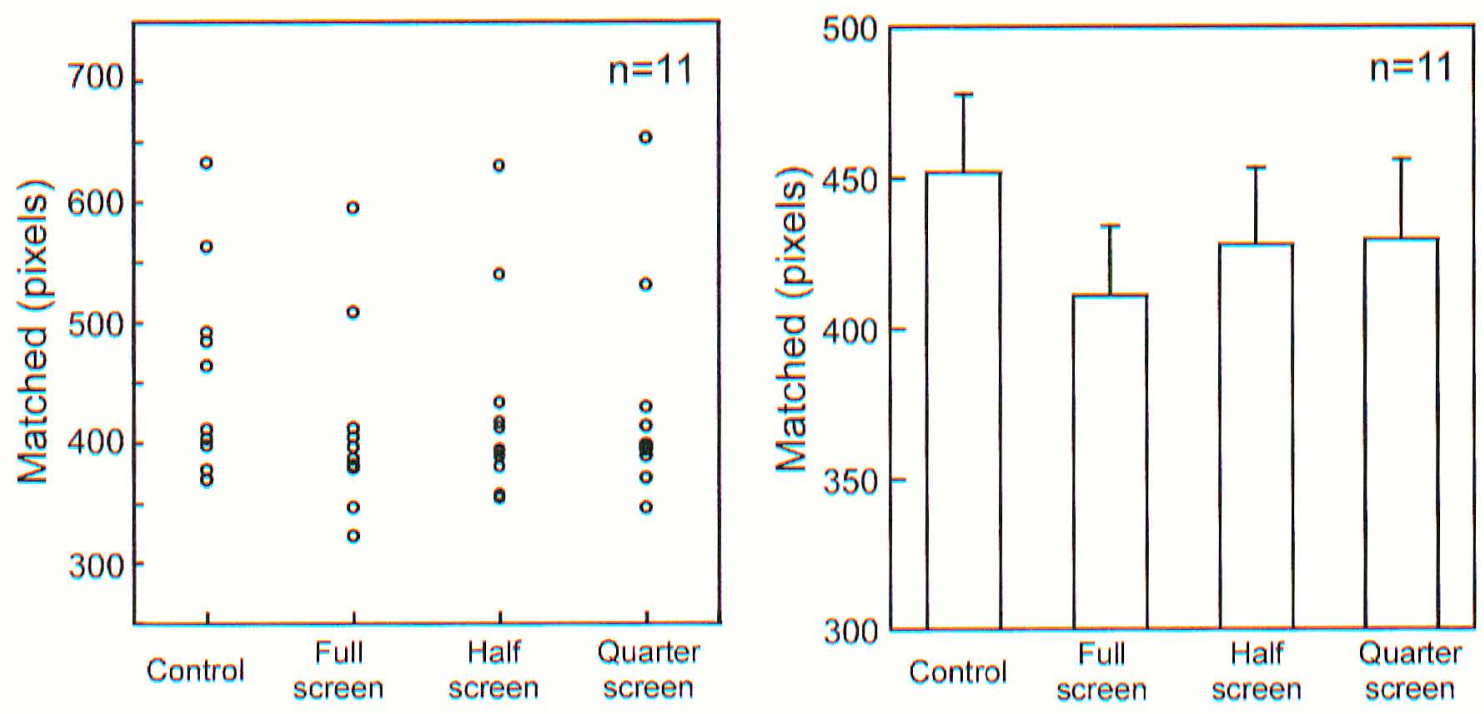

(b)

Figure 7.6. Distance judgment with different amount of occlusion. (a). The screen snapshots of the stimuli. (b). Left: subjects' judgments of distance in four conditions. Right: the effect as a percentage of the mean judged distance. Error bars represent $1 \mathrm{SE}$. 


\section{CURRICULUM VITAE}

NAME: $\quad$ Bing Wu

ADDRESS: Dept. of Psychological \& Brain Sciences

Univ. of Louisville

Louisville, KY 40292

DOB: Qianjiang County, Hubei, China - June 28, 1973

EDUCATION

\&TRAINING: $\quad$ B.S., Biomedical Engineering

Huazhong Univ. of Science \& Technology, China

1990-1994

M.S., Neurobiology

Shanghai Institute of Physiology, Academia Sinica, China 1994-1997

M.S., Computer science

University of Louisville, US

1999-2002

Ph.D., Experimental Psychology

University of Louisville, US

1999-2004

TEACHING: $\quad$ Assistant Lecturer, Undergraduate level teaching.

Dept. of Biomedical Engineering,

Huazhong Univ. of Science \& Technology, Wuhan, China.

1997-1999

PROFESSIONAL AFFILIATIONS: Vision Sciences Society

RESEARCH SUPPORT:

Research funding from Sigma Xi, the Scientific Research Society, "Space vision based on texture information in real and virtual environments", \$350, 2001-2002. 


\section{PUBLICATIONS:}

1. Yang, D.S., Wu, B., Sun, F.C. \& Li, H. (1996). Optokinetic Nystagmus (OKN) in normal and abnormal binocular vision. Acta Biophysica Sinica, Vol. 12 (4), 647-651.

2. Wu, B. \& Sun, F.C. (1999). Eye movements in the recognition of rotated Chinese characters. Acta Psychologica Sinica, Vol. 31 (1), 7-13.

3. Ooi, T.L., Wu, B. \& He, Z.J. (2001). Distance determined by the angular declination below the horizon. Nature, 414, 197-200.

4. Wu, B., Ooi, T. L. and He, Z. J. (2004). Perceiving distance accurately by a directional process of integrating ground information. Nature, 428, 73-77

5. He, Z. J., Wu, B., Ooi, T. L., Yarbrough, G. \& Wu, J. (2004) Judging egocentric distance on the ground: occlusion and surface integration" Perception, (in press).

6. Wu, B., Ooi, T.L., \& He, Z.J. The ground-based distance perception in the virtual environment, (In preparation).

7. Wu, B., Ooi, T.L., \& He, Z.J. Relative effectiveness of texture cues in absolute distance judgment, (In preparation).

8. Ooi, T.L., Wu, B., \& He, Z.J. The direction constancy rule in space perception, (In preparation).

\section{NATIONAL MEETING PRESENTATIONS (PEER REVIEWED)}

1.Wu, B, Ooi, T.L. \& He, Z.J. (2000). Perceived object's location in the dark is not veridical, but not fortuitous. ARVO Annual Meeting, May 2000 (Abstract \# 1194).

2.He, Z.J., Wu, B \& Ooi, T.L. (2000) Non-vertical distance perception due to false perspectives. ARVO Annual Meeting, May 2000 (Abstract \# 3857).

3.Wu, B., He, Z.J. \& Ooi, T.L. (2001). Efficient attention shift on a common surface in 3D: Evidence from eye movements. ARVO Annual Meeting, May 2001 (Abstract \# 4648).

4.Wu, B., He, Z.J. \& Ooi, T.L. (2001). Judging absolute distance by relying on linear perspective and texture density cues. VSS Annual Meeting, May 2001 (Abstract \# 381).

5.Ooi, T.L., May, K.A., Gunther, P.J., Wu, B. \& He, Z.J. (2001). Prism Adaptation Effects On Absolute Distance Judgment. VSS Annual Meeting, May 2001 (Abstract \# 5).

6.Wu, B., He, Z.J. \& Ooi, T.L. (2002). A ground surface based space perception in the virtual environment. VSS Annual Meeting, May 2002 (Abstract \# 516).

7.Yarbrough, G.L., Wu, B., Wu, J. He, Z.J. \& Ooi, T.L. (2002). Judgments of object location behind an obstacle depend on the particular information selected. VSS Annual Meeting, May 2002 (Abstract \# 630).

8.Ooi, T.L., He, Z.J., \& Wu, B. (2002). Delineating the perceived ground surface from a direction constancy rule. VSS Annual Meeting, May 2002 (Abstract \#721).

9. Wu, B. He, Z.J. \& Ooi, T.L. (2003). Evidence for a sequential surface integration process from judging egocentric distance with restricted view of the ground. VSS Annual Meeting, May 2003 (Abstract \#SU25). 